\title{
A Mobile Engineered Tactile Modulation Platform to Recreate TaCtILE FEedBack IN UPPER-LIMB PROSTHESES
}

A Thesis
Presented to
the faculty of the School of Engineering and Applied Science
University of Virginia

in partial fulfillment

of the requirements for the degree

Master of Science

Sarah Lightbody

May

2014 


\section{APPROVAL SHEET}

The thesis

is submitted in partial fulfillment of the requirements

for the degree of

Master of Science

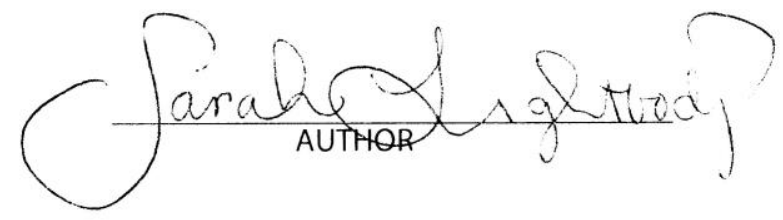

The thesis has been read and approved by the examining committee:

Gregory J. Gerling

Advisor

Stephanie Guerlain

Stephen D. Patek

Accepted for the School of Engineering and Applied Science:

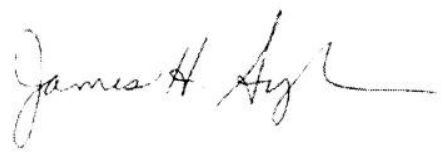

Dean, School of Engineering and Applied Science

May

2014 


\section{TABLE OF CONTENTS}

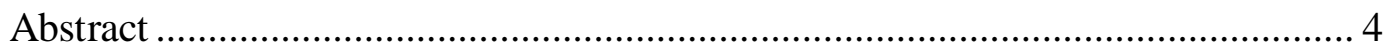

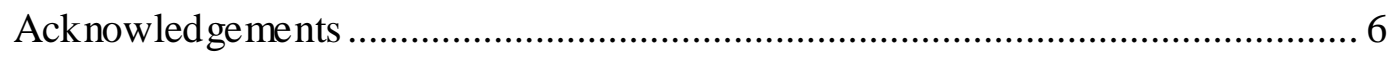

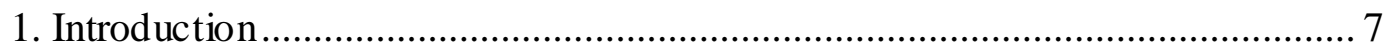

2. Background of Known Phenomenon in Peripheral Afferents ......................... 11

2.1 Force Sensor Phenomenon: Between and Within Sensor Variability .... 11

2.2 Nerve Phenomenon: Absolute Detection Thre shold ............................. 12

2.3 Nerve Phenomenon: Discrimination Sensitivity ................................ 13

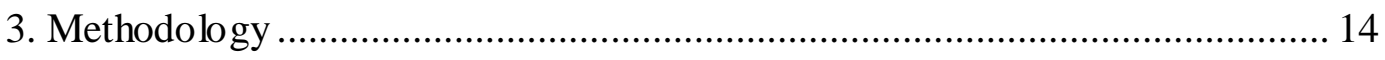

3.1 Part 1: Signal Transformation Apparatus ............................................... 15

3.1.1 Concept of Operations ............................................................... 15

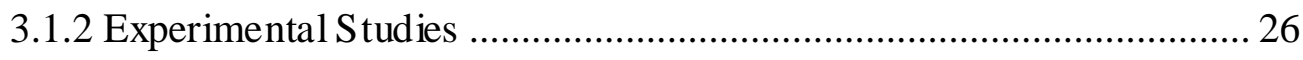

3.1.3 Data Analysis Methodology …...................................................... 31

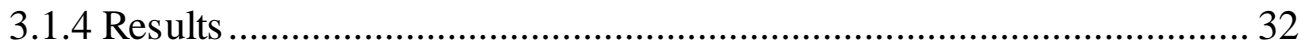

3.2 Part 2: Calibration Proced ures and User Interface Design ....................... 41

3.2.1 Force Sensor Calibration............................................................. 42

3.2.2 Nerve Absolute Threshold Calibration .............................................. 43

3.2.3 Nerve Discrimination Sensitivity Calibration.................................... 45

3.2.4 Preliminary User Interface Design.................................................. 47

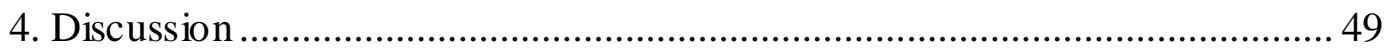

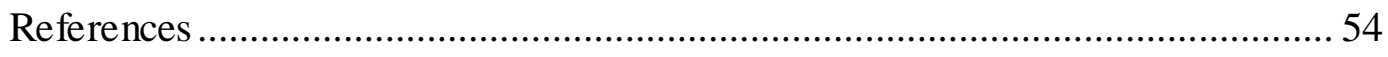

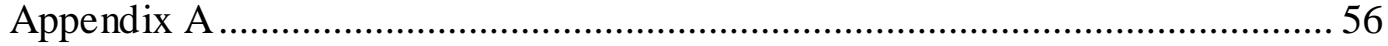

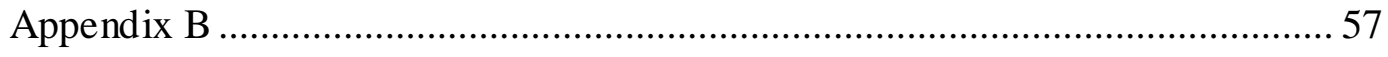

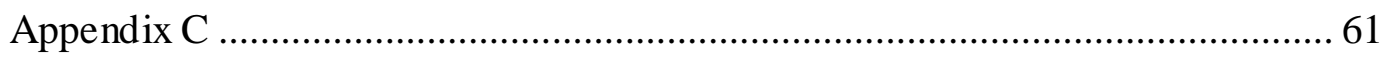

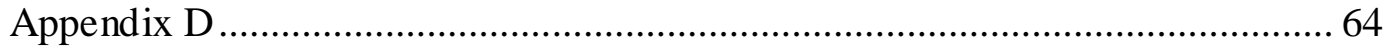

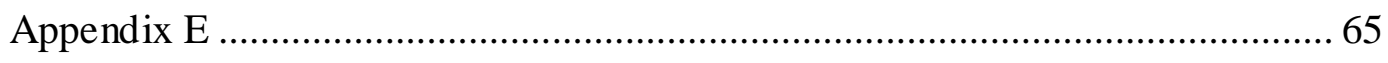

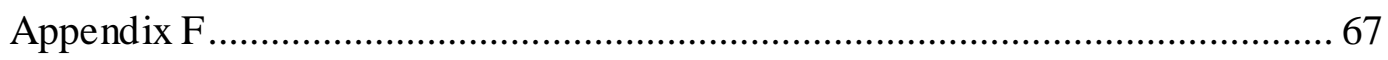

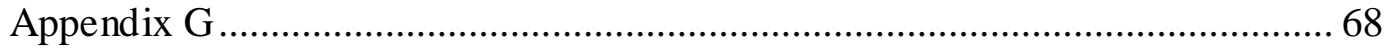

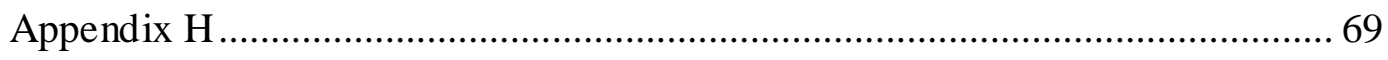




\begin{abstract}
The lack of tactile feedback in grasping objects is one barrier preventing the widespread adoption of upper-limb motorized prostheses. In its absence, amputee users visually attend to graspers, but this creates a high cognitive demand. Efforts to restore tactile sensation require nerve interfaces that use artificial force sensors and electrical stimulation to evoke ionic biological responses. The work herein contributes to the design and testing of a tactile modulation platform that employs models of biological mechanotransduction to better translate force applied at a sensor into biphasic current pulses to be delivered to peripheral afferents, which is done at present in an ad hoc fashion. In specific, the platform consists of a leakyintegrate-and-fire software algorithm of the neuron that generates the timing of the trains of biphasic current pulses, and custom hardware circuitry to control the amplitude and duration of individual pulses. Because of known, naturally occurring phenomenon in the nervous system as well as man-made sensors and electrodes, the platform's parameters need to be calibrated to enable long-term use. To achieve this, procedures and a user interface were designed for use with a mobile phone to afford an intuitive means of adjustment for amputee users with no means to understand the mathematics. Experimental studies were conducted to compare the platform's predicted firing rate to prior electrophysiological recordings in the sural nerve of the rat by applying three levels of force $(1.5,4$, $7.5 \mathrm{~N}$ ), in the nominal case. Then, parameters related to i) nerve discrimination sensitivity were varied for comparison to recorded data for three rats with different stimulus-response transformations, ii) sensor gain were adjusted to
\end{abstract}


account for the variability among three force sensors, and iii) nerve absolute threshold were varied to achieve different biphasic pulse amplitudes. The results indicate that firing rates generated compare favorably to those observed, for both the $0.5 \mathrm{sec}$ duration dynamic ramp-up $(11.2,28.5$, to $42.9 \mathrm{spikes} / \mathrm{sec})$ and the static hold between 2 and $5 \mathrm{sec}$ of the stimulus (7.5, 14.5, to $30.1 \mathrm{spikes} / \mathrm{sec})$. In addition, the platform could account for the both more and less steep sensitivity functions of three additional rats, producing static phase firing rates spanning 6.7 - 129.8 spikes/sec. Three force sensors were standardized to a load cell (standard of comparison) with linear goodness of fit values above 0.9 and estimated force compared to actual force with all root mean square errors below 1. Finally, the platform was able to produce amplitudes of biphasic pulses over a range of \pm 0.2 $15 \mathrm{~V}$. 


\section{ACKNOWLEDGEMENTS}

This work was sponsored by the Defense Advanced Research Projects Agency administered by the Microsystems Technology Office under the auspices of Drs. Jack Judy and Doug Weber through the Space and Naval Warfare Systems Command under grant N66001-11-C-4190. The views expressed in this work are those of the authors and do not necessarily reflect the official views of DARPA.

The invaluable guidance and recommendations offered by the advisor, Dr. Greg Gerling, along with the advisory committee, Dr. Guerlain and Dr. Patek, were instrumental to the formulation of this work.

The author would like to thank Dr. Paul Cederna, Dr. Melanie G. Urbanchek, and Dr. Nicholas B. Langhals for providing valuable insight into the platform design and fostering valuable conversations. Other thanks to Dr. Kris Sugg for the collection of the electrophysiological data, without which this platform would have been impossible to design.

Additionally, the author would like to thank Sean Gallahan for his collaboration with using the indenter, Shawn Wang for his expertise using Lab View, along with Vince Pulido, Jessica Vermaat, Lawrence Bonczar, and Lindsay Wang for support throughout.

Finally, the love, encouragement, and support from Gary and Susan Lightbody made this endeavor possible. Thank you for teaching me that hard work, dedication, and perseverance triumphs both talent and luck. You have the capability to accomplish whatever your dreams may be. Follow them. -Mom 


\section{INTRODUCTION}

Recent, motorized upper limb prostheses have witnessed great strides in neuromuscular control directly from nerves and also from existing muscles, while somatosensory feedback (i.e., tactile and proprioceptive) remains largely undeveloped. Somatosensory feedback is vital to the operation of prosthetics, and a lack thereof remains a major reason why motorized prostheses, especially advanced versions, are rejected [1, 2]. Without such feedback, a user faces high cognitive workload in maintaining visual attention to the operation of his or her motorized grasper or actuated joint. Tasks of everyday living, such as holding a cup of coffee or opening a door, require sensory feedback [3]. Introducing somatosensory feedback is one part of a larger effort to improve advanced prostheses technology as a whole. One hurdle many researchers are currently addressing is in how to interface the nervous system without causing permanent damage, such as biofouling. Addressing this issue requires a careful consideration of how and where electrical stimulation is delivered [4].

Electrical signals delivered to central or peripheral nerves cause depolarization and evoke a neural ionic response [5-8]. To provide input to the nature of the electrical stimulation, physical sensors, serving as a surrogate to mechanoreceptive end organs that normally inhabit the skin, convert forces encountered through interaction with environmental stimuli into time-varying analog voltage. Then, the output of artificial sensors is algorithmically transduced into trains of biphasic, charge balanced pulses (biphasic pulses) to be used as input to a bundle of nerves or neuron. A higher magnitude of force relates to a 
higher frequency of biphasic pulses, as does a higher rate of force. Given the input of biphasic pulses, the membrane potential is crossed and a single nerve fiber's cell membrane is depolarized due to rapid opening and closing of voltage-ion gates within the cell membrane. This rapid influx of sodium and subsequent outflow of potassium ions is characterized as an action potential and can be recorded by a more proximally located electrode. When one records from a bundle of fibers of a large nerve, the action potentials of several fibers are observed, roughly by superposition, in the form of a compound sensory nerve action potential (CSNAP).

Electrical stimulation of peripheral nerves biphasic pulses has shown success in mimicking touch sensation $[9,10]$. This research shows that ad hoc stimulation (linearly relating force magnitude to pulse frequency [9]) can elicit a sensory percept that aligns with the subjective experience of increasing pressure. That said, moving toward a regime of better controlled stimulation is a goal, and there are three practical limitations in electrically stimulating the peripheral nervous system, the: 1) restriction to stimulating the whole nerve (or partial, at least 10 fibers) instead of single fibers due to the nature of nerve to electrode interfaces [16], 2) infeasibility of a long-term closed loop system, whereby one might be able to stimulate and record from the same nerve, which limits validation, and 3) critical need to limit electrical current delivered to the nerve to avoid permanent damage, yet be sufficient to elicit a response. We suggest herein that the next step is to better align both the algorithms and their parameters with neurophysiological processes. 
Mathematical models have been developed to characterize the stimulus-response functions of various cutaneous afferent types, such as the slowly-adapting type I (SAI) [11] and the dynamically-sensitive rapidly adapting (RA) afferents [12]. The SAI afferent is of particular interest to touch perception because of its sustained response to held stimuli over the course of minutes, in addition to response to moving stimuli over a wide variety of velocities and vibrational frequencies. Its high density $\left(100 \mathrm{percm}^{2}\right)$ in the skin makes its populations highly capable of discerning spatial edges, corners, and curvature [13-15]. Lesniak and Gerling's model of the SAI afferent produces the timings of spike elicitation for held stimuli, utilizing a sigmoid transduction function to model transmembrane current and a leaky integrate-and-fire model of neuronal dynamics [11]. Other efforts focus on vibratory stimuli, where models of mechanoreceptors employ information about stimulus position and its $1^{\text {st }}, 2^{\text {nd }}$, and $3^{\text {rd }}$ derivative to predict the precise spike timing of responses from SAI, RA, and Pacinian (PC) afferents [12]. Models that predict the spike response times to the magnitude and rate of force are most useful for mimicking stimuli related to the basic operation of advanced prostheses.

Until recently, electrical stimulation and afferent modeling efforts have traveled parallel, but not intersecting, paths. Works of E. K. Kim and S. Bensmaia both provide a system to transform controlled ramp and hold forces into the timings of when to stimulate the nerve $[12,16]$. Although this work bridges the gap between electrical stimulation and modeling, the algorithm of Kim is essentially a linear relationship between force and pulse frequency, akin to the ad hoc model of 
Dhillon. In contrast, while a neurophysiological representation of nerve transduction, the Bensmaia model does not produce biphasic pulses to overlay upon the timings. Neither system is mobile or easily or practically reconfigurable.

In implementing a more biologically-based receptor model on a mobile platform, it is evident that an intuitive means to adjust the algorithm's parameters will be needed. The parameters will need to be adjusted to account for several known phenomena, for example, the nerve absolute detection threshold varies between subjects and it is hypothesized that short-term variability of absolute thresholds of detection may exist due to natural fluctuations of the body. Additional known phenomenon include within sensor variability over time, between sensor variability, as well as sensitivity to differentiate levels of stimulus magnitude. Calibrating parameters mapping to such phenomenon would allow for consistent signal delivery in experimental and eventually clinical settings.

The objective of this work is to build and test a mobile, tactile modulation platform that employs models of biological transduction to deliver electrical stimulation to nerves based on forces sensed. Second, because of known, naturally occurring phenomenon in the nervous system as well as man-made sensors and electrodes, platform parameters need to be calibrated over time, for long-term use. To achieve this, we design the system in the context of a user-interface designed for a mobile phone to afford an intuitive means of adjustment for users with no programming or mathematical background. 


\section{BACKGROUND OF KNOWN PHENOMENON IN PERIPHERAL AFFERENTS}

An understanding of known, naturally occurring phenomenon in peripheral afferents aids the design of an engineered tactile modulation platform by identifying areas prone to variability. In the engineered design, three specific phenomenon are incorporated: 1) force sensor variability both between sensors and within a sensor over time, 2) nerve absolute detection threshold, and 3) nerve discrimination sensitivity.

\subsection{ForCE SENSOR PHENOMENON: BETWEEN AND WITHIN SENSOR}

\section{VARIABILITY}

It is well known that the input-output relationships vary between sensors, as shown with a small sample of three sensors (Figure 1). This figure shows the difference in force recorded for the hold phase of a stimulus by sensors ( $\mathrm{x}$-axis)

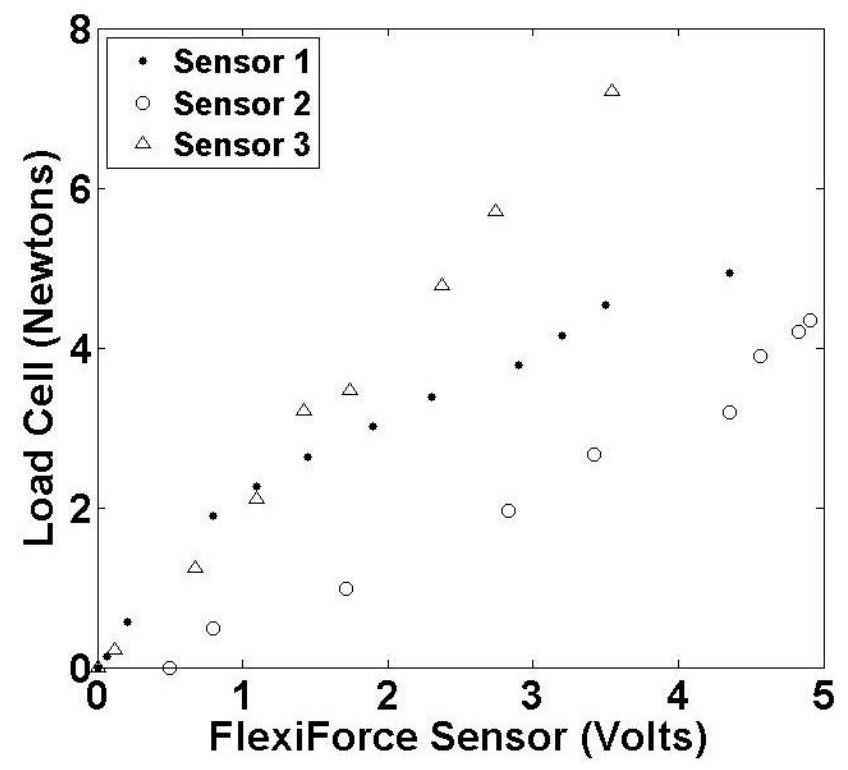

Figure 1: Voltage output from three different FlexiForce sensors ( $x$-axis) demonstrates between-sensor variation from the true force value (Newtons) recorded from a load cell ( $y$ axis). though each FlexiForce sensor itself is annroximatelv linear. 
and by a load cell (y-axis) over 0-7 N. Likewise, drift has been observed withinsensors, over durations of time in weeks or months, making it necessary to utilize calibration procedures to ensure consistent sensor output.

\subsection{NerVE PHENOMENON: ABSOLUTE DETECTION THRESHOLD}

The lowest amplitude of biphasic pulses to eliciting a single CSNAP has been observed to vary between subjects (Figure 2). Two conditions should be met in an engineered system: 1) when a biphasic pulse is delivered, there should exist great confidence (> 99\%) that it will elicit a CSNAP response from the nerve, and 2) no more electrical charge than that should be delivered, in order to preserve the health of the nerve. The stimulation amplitude required to elicit consistent CSNAP response was observed to vary between 0.3 and $0.5 \mathrm{~mA}$ in rat experiments. For example, in recording from the whole sural nerve of one rat, which was stimulated, amplitude was experimentally increased in steps until a CSNAP was reliably observed above a threshold of three sigma above noise, a

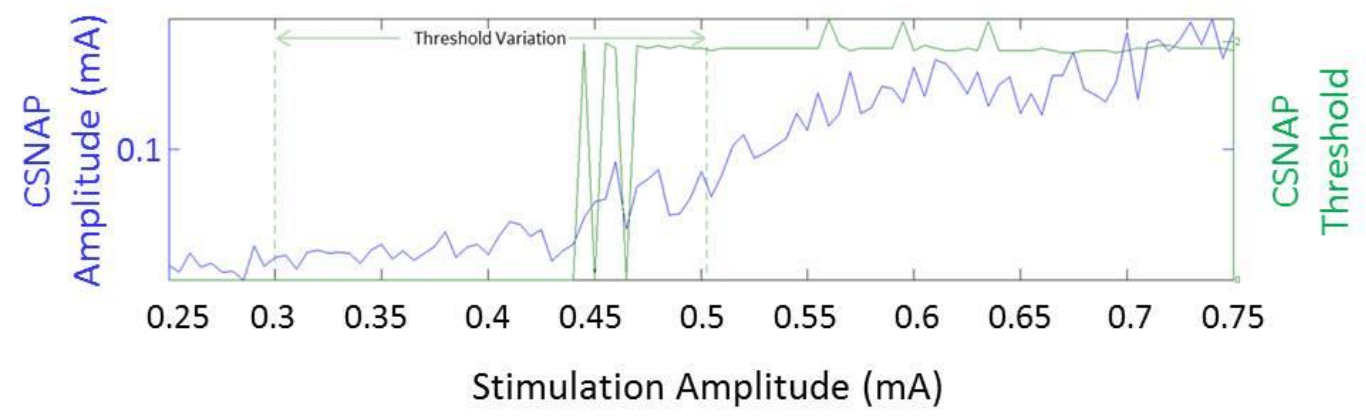

Figure 2 The amplitude of current at which a nerve is stimulated must be large enough to consistently elicit a CSNAP response. When a response is defined as three sigma above the recording's noise, this is referred to as CSNAP threshold (green solid line) and was observed to be about $0.46 \mathrm{~mA}$ in an experiment with a single rat (shown). Across a number of rats, the range of this threshold may vary, as denoted by the label "Threshold Variation." (Sugg, unpublished) 
stimulation amplitude of $0.46 \mathrm{~mA}$ was required (Figure 2, green). Note however, that stimulation amplitudes may vary even more greatly given different electrode materials, sizes and positioning on either nerve or muscle.

\subsection{NERVE PHENOMENON: DISCRIMINATION SENSITIVITY}

Firing rates vary considerably between subjects as a function of applied force, as observed in Figure 3. In this example, the firing rate for Rat 1 increases twice as fast as that for Rat 2. Distinguishing this difference between subjects allows for each to distinguish between varying levels of applied force.

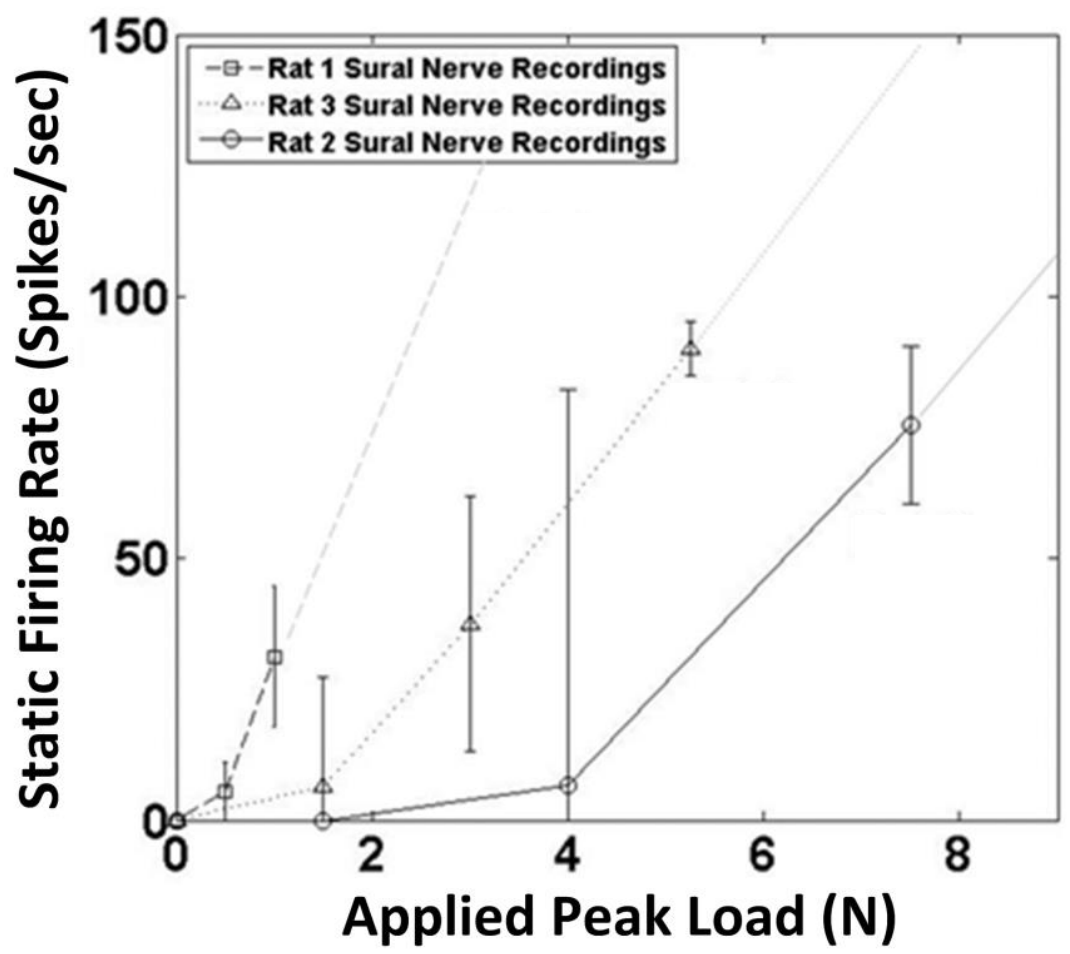

Figure 3: Firing rate response to applied force in the static hold phase of the stimulus varies between three rats, where black denotes collected data from sural nerve recordings in the rat. Because data collection did not span the entire range of sensitivity for each rat, data projections (dotted line) depict expected firing rates for force magnitudes above those where data were collected (Sugg, unpublished). 


\section{MethodOLOGY}

The tactile modulation platform herein was designed with a signal transformation apparatus to convert force stimuli into trains of biphasic pulses mimicking the response of a SAI afferent, and a set of behavioral procedures whereby the apparatus' underlying parameters can be intuitively adjustable by users.

First, the signal transformation apparatus was comprised of a force sensor conditioning circuit to smooth detected force input to a leaky-integrate-and-fire based mathematical model that output pulse timings, before being transformed into biphasic pulses via a signal transformation circuit. In relation to the signal transformation apparatus, four experimental studies were performed to test the impact of varying parameters relating to: sensor gain, nerve discrimination sensitivity, and absolute threshold detection, and collect output data: goodness of fit, dynamic and static firing rate, and biphasic pulse amplitude, respectively, for comparison to known data sets recorded in the rat, a nominal case and three additional rat data sets that varied in terms of nerve sensitivity.

Second, taking a systems approach that looked forward to future deployment of a system that would need to be calibrated and maintained in consultation with a physician, the design of the user interface and procedures was done to tie parameters of the signal transformation apparatus directly to the three known phenomena. These procedures drove the selection of parameters, but only represent a preliminary design and do not represent the focus of the thesis herein. 


\subsection{Part 1: Signal Transformation Apparatus}

\subsubsection{CONCEPT OF OPERATIONS}

The Tactile Modulation Platform is composed of two components: a) signal transformation apparatus and b) calibration procedures designed for a graphical user interface. The signal transformation apparatus was designed to account for parameter adjustment and calibration as to mimic naturally occurring phenomenon. The parameters selected for adjustment affect the frequency and amplitude of the biphasic pulse output and account for three known phenomenon: 1) force sensor, 2) nerve absolute detection threshold, and 3) discrimination sensitivity. This effort was synchronous with the procedures for a graphical user interface design to provide a medium for intuitive parameter adjustment via procedures, which will be discussed in Section 3.2. To transform force into an appropriate train of biphasic pulses for delivery to the nerve, several data transformations are made by the signal transformation apparatus. The first occurs at the site of stimulation, where a force sensor transduces applied indentation (Figure 4 Input, Figure 5A). From the voltage, force is calculated based on sensor calibration parameters $m$ and $b$ (Figure 4 Force Sensor Signal Conditioning, Figure 5B). 


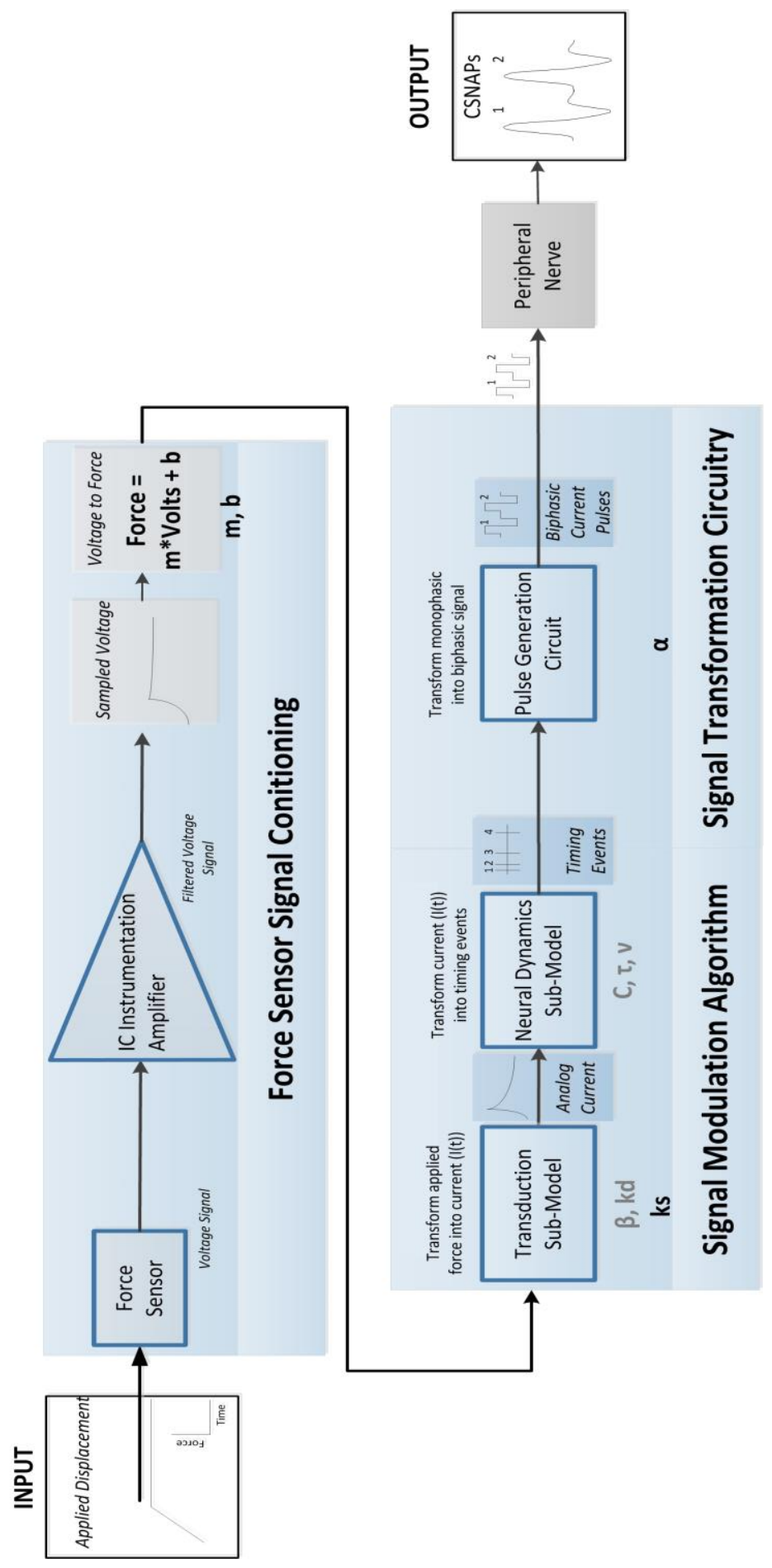

Figure 4: Diagram of the Platform, where displacement recorded by a sensor inputs to Force Sensor Signal Conditioning, where the signal is then transformed from voltage into force and is the input to the Signal Modulation Algorithm, and output current pulse timings are input to the Signal Transformation Circuitry, which produces the final current limited, charge balanced pulse appropriate to deliver to the sural nerve. User Calibration Procedures performed through a Graphical User Interface allow the user to adjust system parameters. 

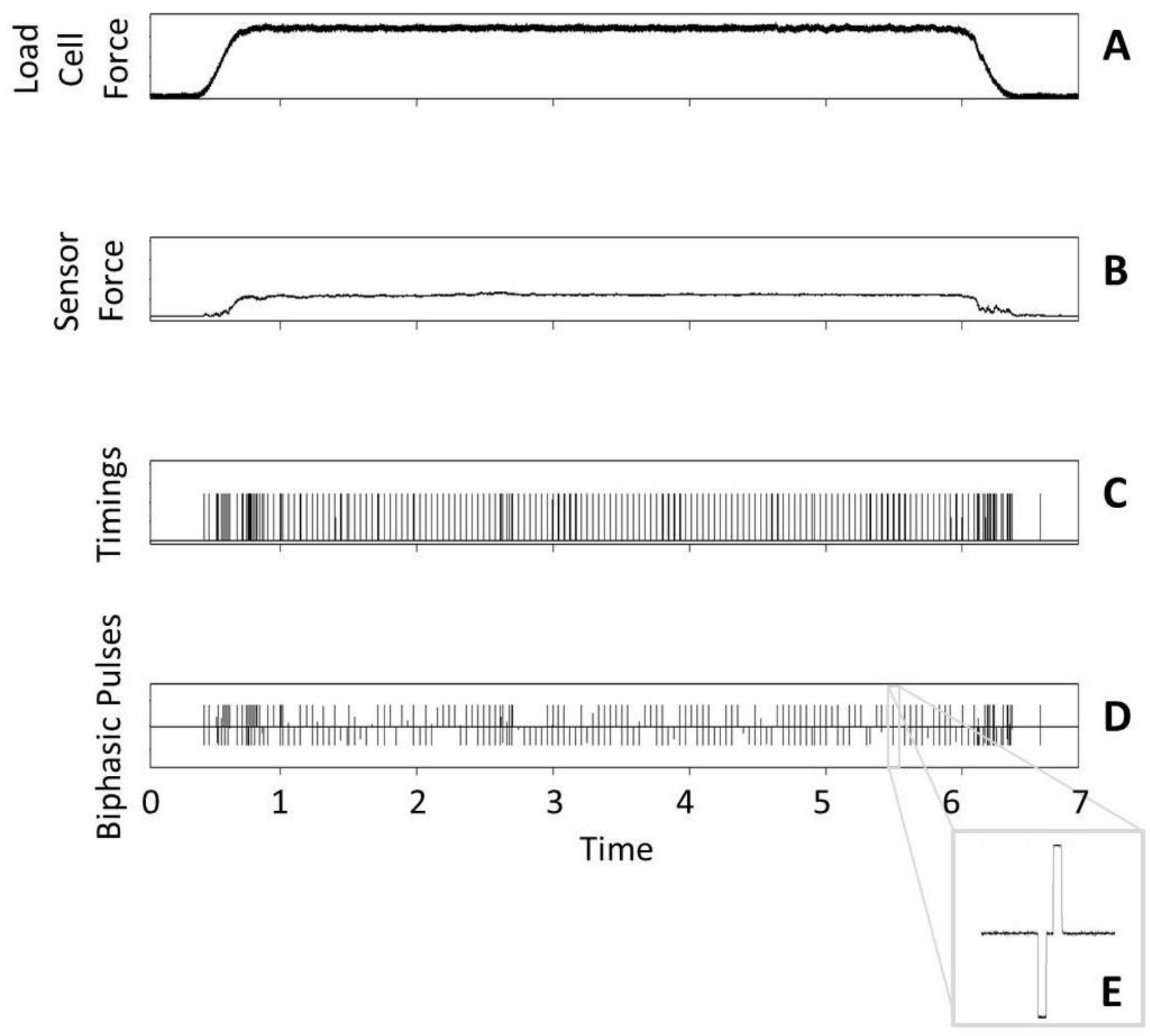

Figure 5: Displacement applied to the sensor-substrate is recorded as force at the load cell (A) and at the embedded sensor (B). The current pulse timings output from the Signal Modulation Algorithm (C, correspond to biphasic pulses (D) generated by the Stimulation Isolation Circuitry with a 1:1 ratio. Due to the LabView sampling frequency limitations, only partial biphasic pulses are captured in the data collection. However, a triggered biphasic pulse is shown for representation (E). Note time is measured in seconds. 
The force signal is the input to the Signal Modulation Algorithm, where parameters $\beta, k_{s}, k_{d}, \mathrm{C}, \tau$, and $\bar{v}$ affect how force is transformed first into trans membrane current (Transduction Sub-Model), and then into current pulse timings (Neural Dynamics Sub-Model) (Figure 4, Signal Modulation Algorithm, Figure 5C). The Signal Transformation Circuitry creates appropriate biphasic pulse trains based on the input of current pulse timings from the Signal Modulation Algorithm through a Pulse Generation Circuit, where parameter $\alpha$ affects current pulse amplitude (Figure 5D, 5E).

\section{Force Sensor Signal Conditioning}

To emulate the location of SAI afferents throughout the skin and to account for skin elasticity, a piezo-resistive force sensor (Flexiforce A201; Tekscan, Inc., South Boston, MA) was affixed within a silicone-elastomer substrate using a similar procedure as prior sensor-substrate experiments [16] (Appendix A).

Displacement applied at a substrate is transduced into voltage at the force sensor, such that an increase in applied force results in a decrease in the sensor's resistance. The noise from the signal is reduced and amplified using an IC instrumental amplifier (INA114AP; Burr-Brown Corporation, Tuscon, AZ). Before the signal can be used as input to the Signal Modulation Algorithm, force must be calculated from the voltage signal (Eqn 1). The signal progresses through this process as shown in Figure 6 and is appropriate to input to the Sensory Modulation Algorithm.

$$
\text { Force }_{\text {calculated }}(t)=m * \text { voltag }_{\text {flexiforce }}+b
$$




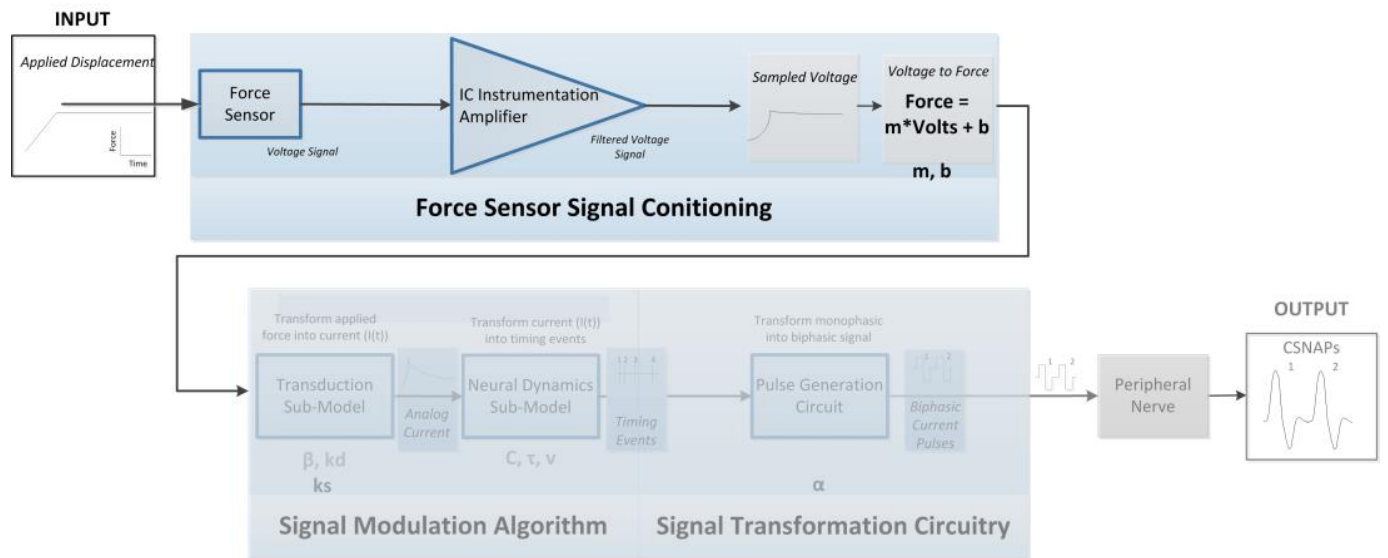

Figure 6: Force applied to a sensor is recorded as voltage, conditioned, and force is calculated using parameters $\mathrm{m}$ and $\mathrm{b}$

The onset latency, or the time lag between applied force (load cell) and detected force (force sensor) at stimulus onset, is approximately 0.1 seconds, while the offset latency is approximately 0.2 seconds (Figure 7). Latent offset noise is observed in the force sensor recording 0.7 seconds (Figure 7, arrow).

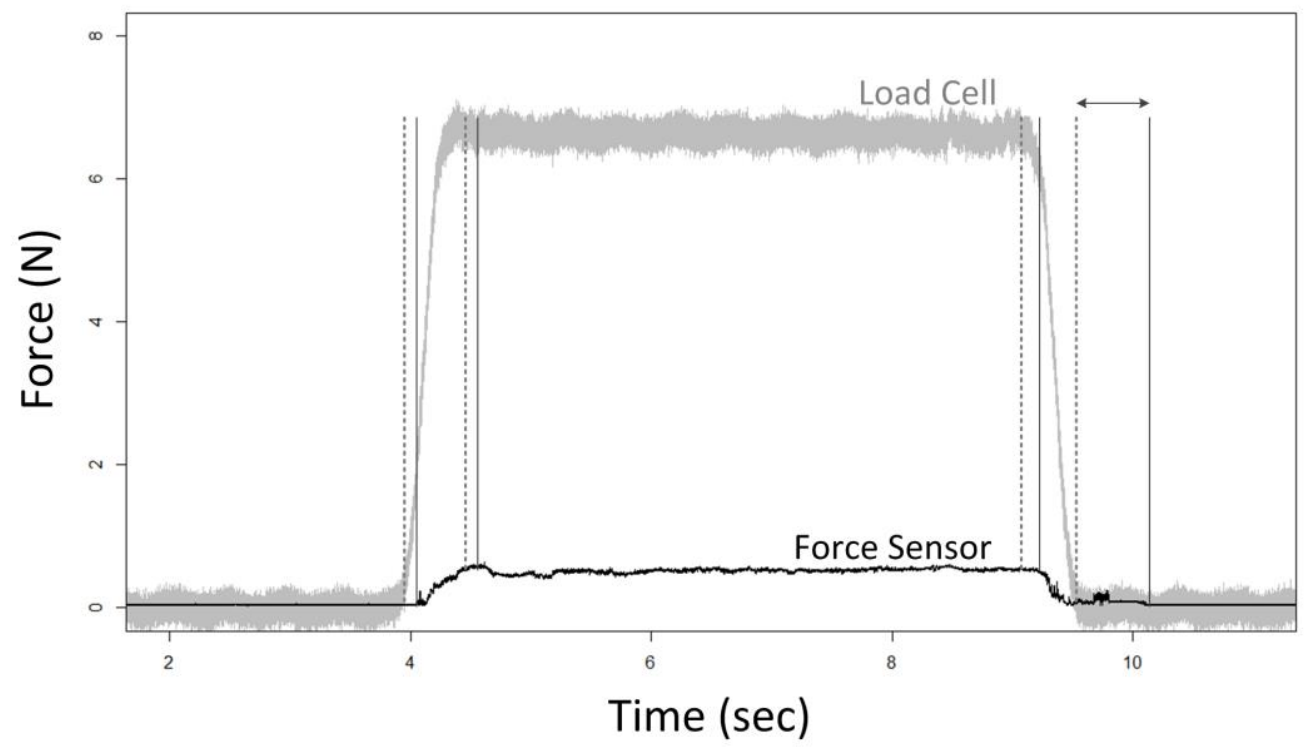

Figure 7: Load cell (grey) and force sensor (black) recordings over time show stimulus detection latencies. Grey dotted lines define the beginning and end of ramp-up and rampdown time periods of applied force (load cell), while black solid lines represent detected force (force sensor). Latent offset noise recorded at the force sensor (arrow) lingers post-stimulus. 


\section{Signal Modulation Algorithm}

A sensory modulation algorithm housed on a micro-controller (Arduino Mega 2560; Arduino, Torino, Italy) uses two sub-models based on the leaky-integrateand-fire (LIF) model to translate sensor detected force into current timings: a neural transduction and neural dynamics model. The neural transduction submodel translates the physical stimulus force, sampled at a $10 \mathrm{kHz}$ collected by the Arduino from the force sensor, into current (Eqn. 2), mimicking the transduction of stress and strain applied at the SAI afferent to current across the membrane. The coefficient terms $\beta, k_{s}, k_{d}$ represent the intercept constant, static gain, and dynamic gain, respectively. This sub-model accounts for both static force, $k_{s} f(t)$, and change in force, $k_{d} f^{\prime}(t)$, allowing for representation of both held and dynamically changing force. The force in this equation is input from the Force Sensor Signal Conditioning portion of the Signal Transformation Apparatus (Figure 8).

$$
I(t)=\beta+k_{s} f(t)+k_{d} f^{\prime}(t)
$$

Skin membrane dynamics are represented using an RC circuit. Current passes through the membrane resulting in the membrane potential, $u(t)$. When this potential, measured in $\mathrm{mV}$, exceeds the membrane threshold, a current pulse is fired. Equation 3 defines mathematically the leaky-integrate-and-fire behavior of an RC circuit, where $\tau$ is the product of membrane resistance $(\Omega)$ and capacitance (C) (Eqn. 4). 


$$
\begin{aligned}
I(t) & =\frac{u(t)}{R}+C \frac{d u}{d t}(t) \\
\tau & =R C
\end{aligned}
$$

Combining these two equations yields a differential equation in which the change in membrane potential is a function of time-dependent membrane potential and current (Eqn. 5).

$$
g(t, u)=\frac{d u}{d t}(t)=\frac{-u(t)}{\tau}+\frac{I(t)}{C}
$$

Using fourth-order Runge Kutta method for solving ordinary differential equations (Eqn. 6-10), we solve for membrane potential by iteratively calculating Eqn.'s 7, 8, 9, and 10. The value of $h$ is $0.01 \mathrm{~s}$, representing the resolution of simulated time. The value of $u(t=0)$ is set to 0 to represent the starting resting potential of the membrane.

$$
\begin{aligned}
u(t+1) & =u(t)+\frac{1}{6}\left(k_{1}+2 k_{2}+2 k_{3}+k_{4}\right) \\
k_{1} & =h * g(t+h, u(t)) \\
k_{2} & =h * g\left(t+h, u(t)+\frac{k_{1}}{2}\right) \\
k_{3} & =h * g\left(t+h, u(t)+\frac{k_{2}}{2}\right) \\
k_{4} & =h * g\left(t+h, u(t)+k_{3}\right)
\end{aligned}
$$




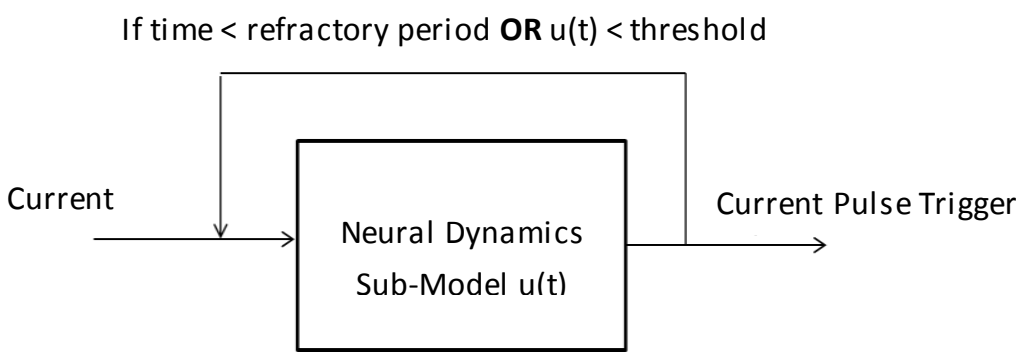

Figure 9: Current enters the Neural Dynamics Sub-Model and is calculated according to equation 6. When the threshold $(v)$ is reached and within the refractory period, a current pulse timing is triggered. If these conditions are not met, the membrane potential $(u(t))$ continues to accumulate, otherwise $u(t)$ is set to 0 and the refractory period begins.

When the membrane potential reaches a threshold, $v$, a current pulse trigger is output from the Arduino. After the trigger has been elicited, the membrane potential is reset to 0 and the model enters an absolute refractory period of $1 \mathrm{~ms}$ in which no current pulse can be elicited (Figure 9). The model implemented in Arduino-based $\mathrm{C}$ is documented in Appendix B.

\section{Signal Transformation Circuitry}

The output current pulse triggers from the Signal Modulation Algorithm are transformed by the Signal Transformation Circuitry (Figure 10) to create trains of charge balanced biphasic current pulses. The pulse generation component [17] transforms mono-phasic pulses into a bi-phasic pulse using three edge-triggered mono-stable multivibrators (SN74121; Texas Instruments, Dallas, TX.). The trigger pulse, generated by the sensory modulation algorithm as a monophasic square wave, is the input to the first multivibrator, which outputs the cathodal portion of the biphasic pulse. The second multivibrator is responsible for creating the inter-phase interval between cathodal and anodal pulses. The anodal pulse is generated by the third multivibrator. The input for the first multivibrator (cathodal 


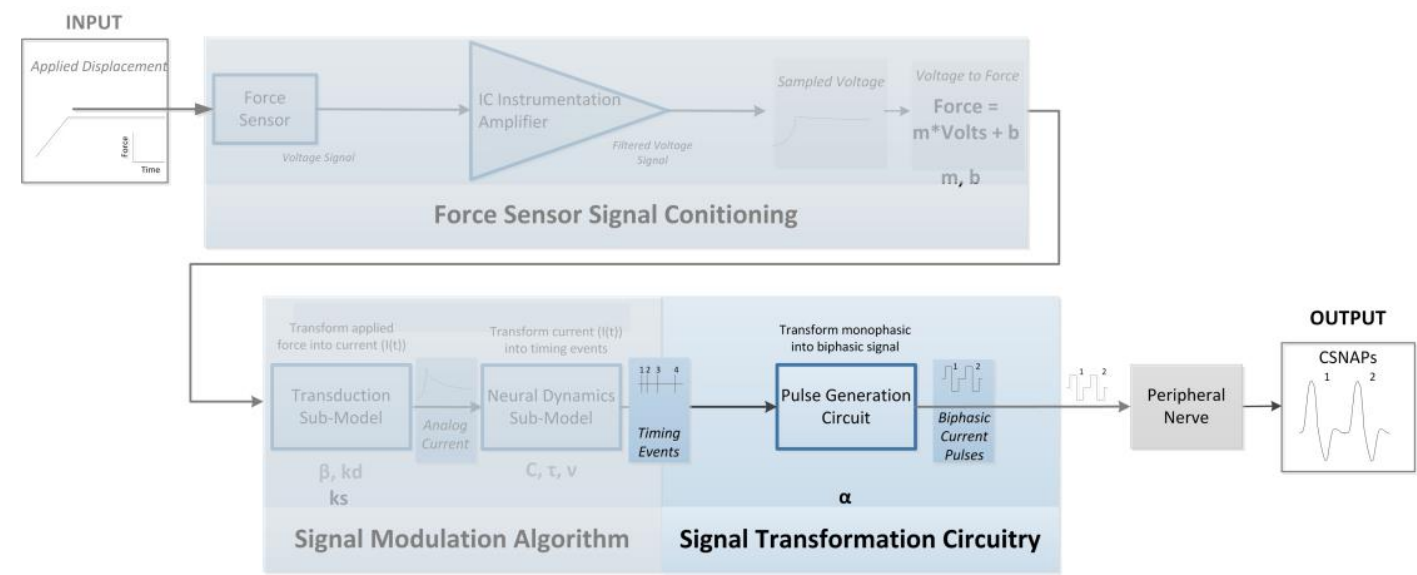

Figure 10: The Signal Transformation Circuitry first transforms the current pulse timings into biphasic pulses (Pulse Generation Circuit) to create a charge balanced pulse deliverable to the sural nerve.

pulse), is the rising edge of the trigger pulse, while the input for the second and third multivibrators (interphase interval, anodal pulse) is the falling edge of each respective input pulse generated from the previous multivibrator. Used in series, the three multivibrators produce timed, digital biphasic pulses. Figure 11 shows the input pulses (A) to the monostable multivibrators (B) and the final biphasic pulse (D) in which the cathodal pulse (1) is inverted by the differential amplifier (C). Each multivibrator in Figure 10B is represented in Figure 12 as 3 "SN74121 'One Shot'" devices. Pulse duration adjustment can be achieved by altering the resistance of the potentiometers denoted in Figure 12 while keeping capacitance constant in the timing portion of each multivibrator circuit. For this application, the pulse duration is constant and resistors used achieve a pulse width of $0.1 \mathrm{~ms}$. The circuit operates at $\pm 15 \mathrm{~V}$ for the dual op-amp and $+5 \mathrm{~V}$ for the monostable multivibrators. Pulse amplitude is adjusted through Serial Peripheral Interfacing with the digital potentiometer (Figure 12, AD 52900). A symmetric reduction of 


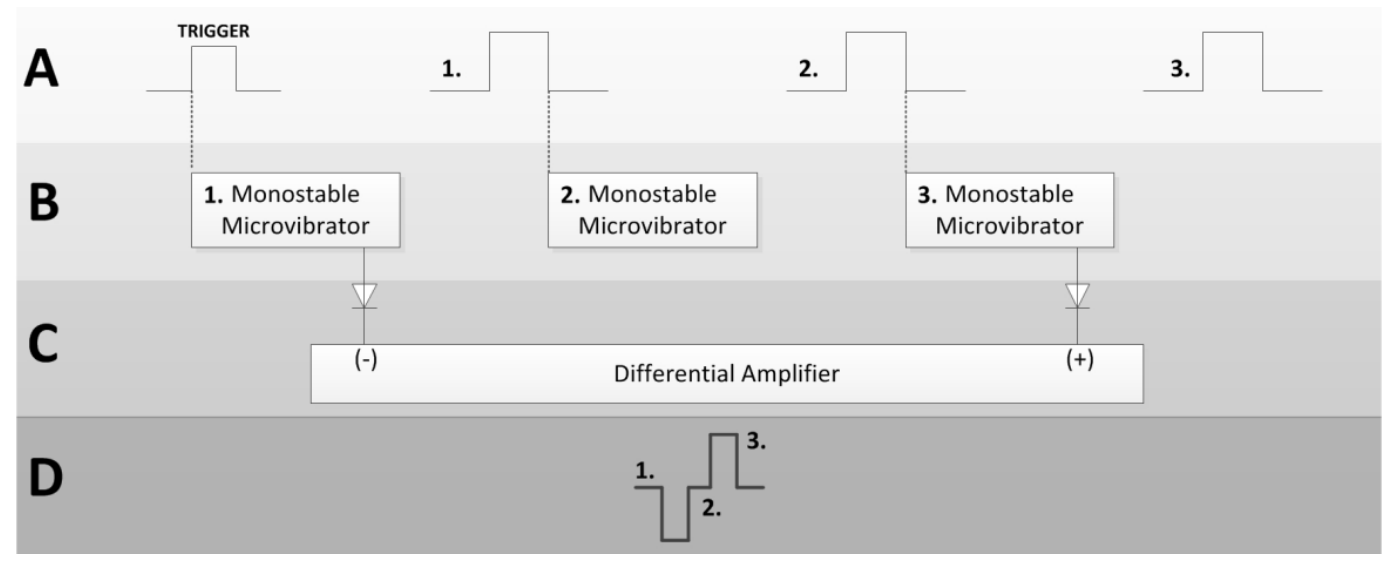

Figure 11: Signal progression through the pulse generation circuit is represented in layers A-D. Triggers (A) for each monostable multivibrator (B) are shown where the leading edge of the input pulse results in an output pulse (A 1.). The falling edge of $A$ 1. triggers the second multivibrators (B 2.). A 2. and B 3. behave similarly. B 1. and B . are inputs to a differential amplifier (C). The biphasic pulse output (D) is obtained by inverting A 1 through C, where D 2 is obtained by the time delay caused by B2.

resistance from the potentiometer controlling the anodal and cathodal portions of the biphasic current pulse has the effect of increasing the pulse amplitude, while an increase in the same manner decreases the pulse amplitude. The supply voltage and digital value of the resistance ( $\alpha$, integers between 1 and 255) alter the amplitude between a range of 1.2 and $15.5 \mathrm{~V}$. The physical layout of the entire tactile modulation platform hardware is documented in Appendix C. 


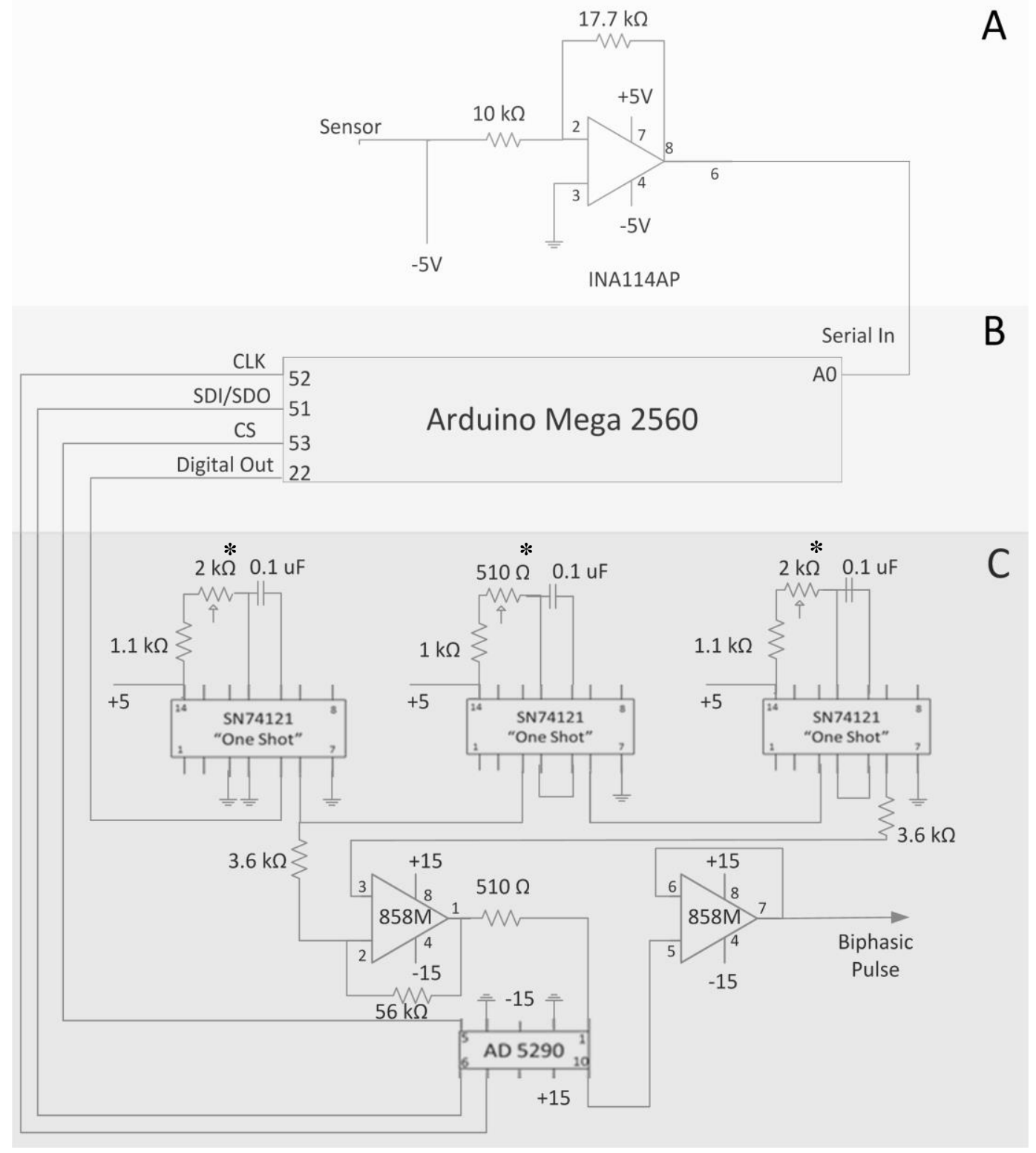

Figure 12: Force sensor conditioning circuit (A) filters and amplifies the signal input to the Arduino (B, pin A0). The Signal Modulation Algorithm outputs current pulse timings from pin 22 (Digital Out). The Pulse Generation Circuit (C), modified from Gwilliam (2008), creates the biphasic pulses, where asterisks highlight the resistors, shown as potentiometers, that control pulse width for the cathodal, interphase interval, and anodal, from left to right, widths of the biphasic waveform. The digital potentiometer (C, AD 5290) receives parameter $\alpha$ values from the Signal Modulation Algorithm using Serial Peripheral Interfacing (B, pins $51,52,53)$ 


\subsubsection{EXPERIMENTAL STUDIES}

\section{Indentation/Force Stimulation and Recording Equipment}

The experiments utilized a custom-built machine to indent a stiff probe into the force sensor embedded in silicone-elastomer. The set-up was similar to that used in a prior experiment [16], where a high-performance motion controller/driver (XPS; Newport Corporation, Irvine, CA) commanded a high-precision, mechanical linear z-stage (UTS Mid-Travel Linear Stages; Newport Corporation). A calibrated load-cell (Sensotec 11 subminiature; maximum load = 22N; OMGEGA Engineering, Inc., Stamford, CT) affixed to the cantilever was used to measure normal force applied to the force sensor silicone-elastomer substrate (Figure 13). The substrate was positioned on a level, aluminum surface directly

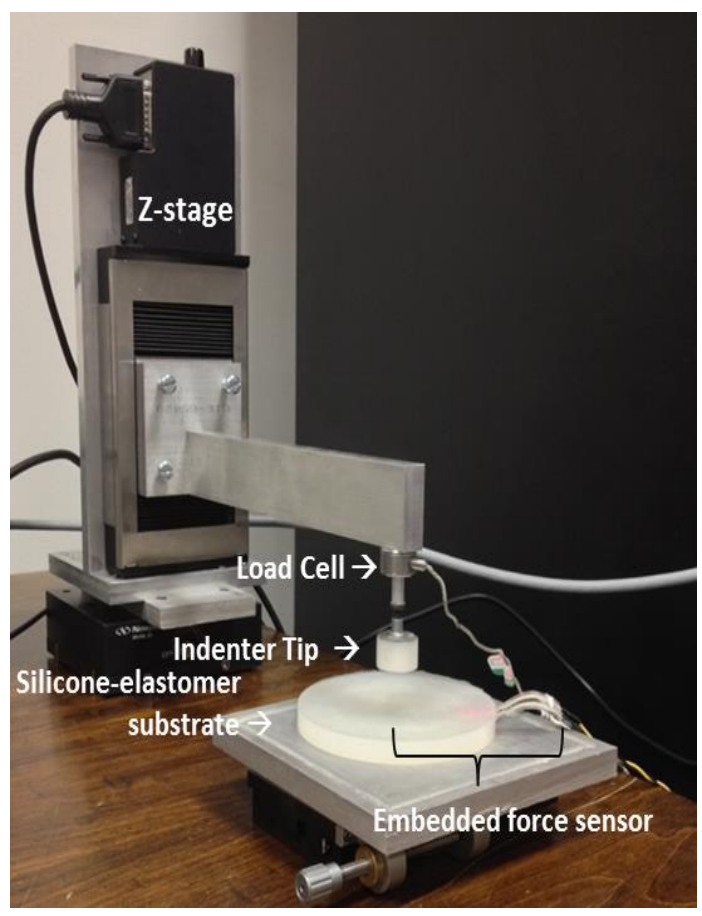

Figure 13: Experimental set up of motion controlled indenter to silicone-elastomer sensor substrate. The motion controller commands the Z-Stage, from which a cantilever maneuvers the indenter tip to provide controlled ramp and hold stimuli. The load cell and embeddec force sensor record information pertaining to applied force. 
below a cylindrical Delrin contractor tip (14 $\mathrm{mm}$ tall by $20 \mathrm{~mm}$ diameter $)$ threaded into the load cell. Centering the substrate to align with the indenter tip ensured an even distribution of force across the receptive area to achieve accurate and consistent recordings [20].

Conditioning exercises preceded data collection, where $110 \%$ maximum load $(8.25 \mathrm{~N}, 1.6 \mathrm{~mm}$ indentation) was applied to the substrate for 10 trials, each of $5 \mathrm{~s}$ duration, to decrease the variability of substrate elasticity between repeated stimulations. Velocity was held constant at $6 \mathrm{~mm} / \mathrm{sec}$ and stimulus duration spanned approximately 5 seconds. Data was collected using a Data Acquisition Board (NI-6221; National Instruments Corporation, Tuscon, AZ) and LabView software during controlled indentation experiments (Appendix D) to record applied force, detected force, current pulse timing triggers, and biphasic current pulse values over time.

\section{Experimental Procedures}

\section{Study 1: Accounting for Sensor Variability through $\mathbf{m}$ and $\mathbf{b}$}

The purpose of this task is to demonstrate the ability of sensory gain parameters to account for the variability between and within sensors. To do this, three force sensors are calibrated with a load cell. The load cell is first calibrated to ensure its accuracy as a standard of comparison. Then, force varying between 0-6 $\mathrm{N}$ was applied to each force sensor through indentation. Plotting the force sensor voltage output values against the load cell force output yields deviations for which $\mathrm{m}$ and b account for. The relationship between applied force and voltage output from the 
sensor is known to be linear, and as such, the force sensor can be fit with the Equation 11, where a linear fitted line provides slope (m), and intercept (b).

$$
\text { force }_{\text {loadcell }}=m * \text { voltage }_{\text {flexiforce }}+b
$$

These same parameters are used in a similar equation to calculate force independent of the load cell (Eqn 2). This equation is shown below for convenience. The extrapolation of calculated force allows evaluation of multiple sensors over the same applied load range.

$$
\text { force }_{\text {calculated }}=m * \text { voltage }_{\text {flexiforce }}+b
$$

Study 2: Biphasic Current Pulse Amplitude Adjustability Demonstration through $\alpha$

Increasing or decreasing the biphasic current pulse amplitude, through the adjustment of parameter $\alpha$, impact the CSNAP waveform amplitude response from the sural nerve in response to biphasic current pulse stimulation. This stimulation to the nerve is dependent upon the type of electrode used, where variations of biphasic charge balanced pulse amplitude output from the tactile modulation platform (V) of at least $5-15 \mathrm{~V}$ produce varying biphasic charge balanced current amplitudes $(0.3-0.5 \mathrm{~mA})$. It is for this reason that no single nominal signal amplitude is desirable, but rather the ability to adjust within the applicable range is desirable.

To demonstrate the flexibility of the platform, $\alpha$ is varied with a step size $h=1$ from $\alpha=1$ to 255 within the Signal Modulation Algorithm. The resolution of $\alpha$ and maximum achievable waveform amplitude is determined by how the digital 
potentiometer is configured. A controlled monophasic square wave input to the Signal Transformation Circuitry triggers the biphasic current pulse signals, which are recorded on an oscilloscope using a compact flash card (Industrial 32MB, Power Quotient International Co., LTD., New Taipei, Taiwan). A data acquisition card would allow for data logging of these experiments, but due to the input limitations of card (+/-10V) and LabView software sampling constraints (maximum $20 \mathrm{kHz}$ ), this method is infeasible. The waveform amplitude is recorded for each variation of $h$ to provide a mapping between $\alpha$ and waveform amplitude.

Study 3: Mimicking SAI Firing Rates in Response to Applied Force for the Nominal Case

The goal of this study is to show the Sensory Modulation Algorithm's ability to recreate firing rates similar to those observed as a nominal case in rats. The parameter $k_{s}$ is directly related to static force, while $k_{d}$ is linked to change in force (recall Equation 2). Here we evaluate the Signal Modulation Algorithm's output in response to applied ramp and hold stimuli.

Ramp and hold is defined as accelerating during the "ramp" period until a peak force is reached, at which point acceleration becomes 0 and force is constant, which is defined as the "hold" period. These stimuli vary in magnitude of peak force. Narrowing the focus to this type of stimuli accounts for varying levels of static force and dynamic force, where the Signal Modulation Algorithm discussed later in this section features a set of parameters directly linked to the input stimulus. 
Here, $k_{d}$ is first manually adjusted to achieve dynamic firing rates consistent with sural nerve recordings (Figure 13A). Ramp and hold displacements $(1.5,4,7.5 \mathrm{~N}$ ) were applied to the silicone sensor substrate, where applied peak loads at the load cell were approximately matched to those used during the sural nerve recordings. Then, $k_{s}$ is adjusted in the same manner to mimic sural nerve static hold firing rates (Figure 13B). After both have been adjusted to approximate the nominal case, 12 trials of each applied peak forces, 36 trials total, were applied to the sensor-substrate and recorded. All trials consisted of a ramp-up phase to which a 5 sec static phase immediately followed, each separated by 25 second rest periods. These periods reduced sensor-substrate adaptation. During the fitting process of both $k_{s}$ and $k_{d}$, the remaining parameters $(\beta, v, \tau$, and $C)$, were held constant at values previously obtained through Response Surface Methodology fitting [16] (Table 1). Although initially this study was prepared to alter these parameters if necessary, such actions were unnecessary as the given values performed the task at hand.

\begin{tabular}{cccc}
\hline \multicolumn{4}{c}{ Fixed Model Parameters } \\
\hline$\beta(\mathrm{mA})$ & $v(\mathrm{mV})$ & $\tau(\mathrm{msec})$ & $\mathrm{C}(\mathrm{mF})$ \\
\hline $2.72 \mathrm{E}-08$ & 47.3 & 71.409 & $9.70 \mathrm{E}-07$ \\
\hline
\end{tabular}

Table 1: Tactile Modulation Platform static parameters. 
Study 4: Varying $\boldsymbol{k}_{s}$ to Mimic Several Cases of Firing Rate Variability Observed in 3 Rats

Here, only $k_{s}$ is be evaluated while the remaining parameters are held constant. As explained previously, for a human-in-the-loop scenario where a recording electrode feedback loop is not available, adjusting parameters $\beta$ and $k_{d}$ becomes impossible in practice. Three nerve cases in rats beyond the nominal case used in Study 3 are used to evaluate varying levels of sensitivity, where the rate at which firing rate increases with increase in applied peak force is a function governed by $k_{s}$.

Using a brute force parameter fitting method $k_{s}$ was systematically altered starting with the base value determined in Study 3. For each iteration, indentation levels are used to obtain similar peak force values from known experimental cases are applied to the sensor-substrate using the same experimental setup and protocol as in Study 3. The resulting current pulse timings are divided into dynamic and static periods and firing rates (FR's) are calculated. Then, the static FR's are compared to the objective case (Rat 1,2, or 3).

\subsubsection{DATA ANALYSIS METHODOLOGY}

Before analyzing the data collected in Studies 2, 3, and 4, independent and dependent metrics were defined before validating the Tactile Modulation Platform's predicted dynamic and static firing rates against those of the sural nerve. Study 1 is not described here as it is a fitting procedure.

Holding the LIF parameters constant, the independent metrics of applied peak force and waveform height parameter $(\alpha)$, elicit changes in the dependent metrics: 
firing rate (FR), or the average number of elicited current pulse timings over time (Eqn 11), for both dynamic (ramp-up) and static (hold) phases and biphasic current pulse height. The dynamic ramp-up time window is defined as the period between stimulus onset and peak detected force while the static hold time window is defined as the period between 2 seconds beyond stimulus onset and 5 seconds into the stimulation (Figure 14). Holding applied peak force constant, the independent metric within the LIF model $\left(k_{s}\right)$ affect the dependent metrics of dynamic and static firing rates.

$$
F R=\frac{N_{\text {total_spikes_per_sec }}}{t_{\text {last }_{\text {spike }}}-t_{\text {first }_{\text {spike }}}}
$$
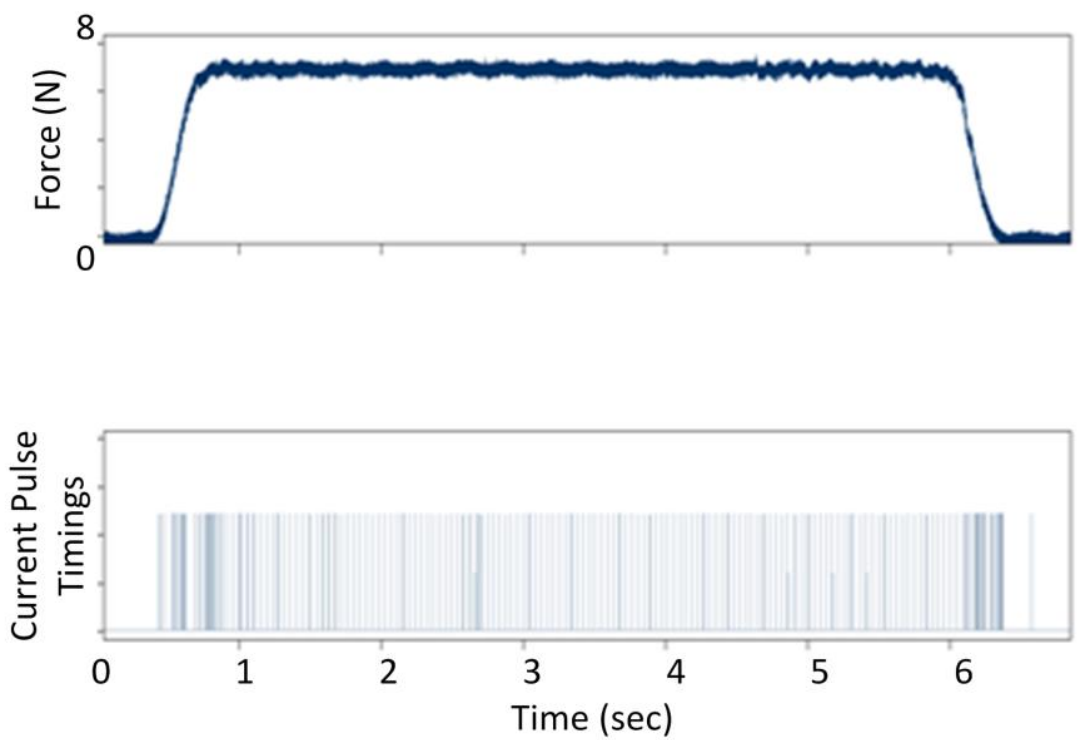

Figure 14: The top graph displays recorded force from the load cell, where dynamic ramp up (A) and static hold (B) periods are defined with gray backgrounds. The bottom graph shows these same time windows with current pulse timing responses from the tactile modulation algorithm. 


\subsubsection{RESULTS}

The following section presents results obtained from each of the four experimental studies.

\section{Study 1: Accounting for Sensor Variability through $m$ and $b$}

The sensors, without being embedded within the silicone-elastomer substrate, all exhibited linearity when calibrated, as expected, and were able to be fit to the load cell standard of comparison with goodness of fit values greater than 0.9. (Figure
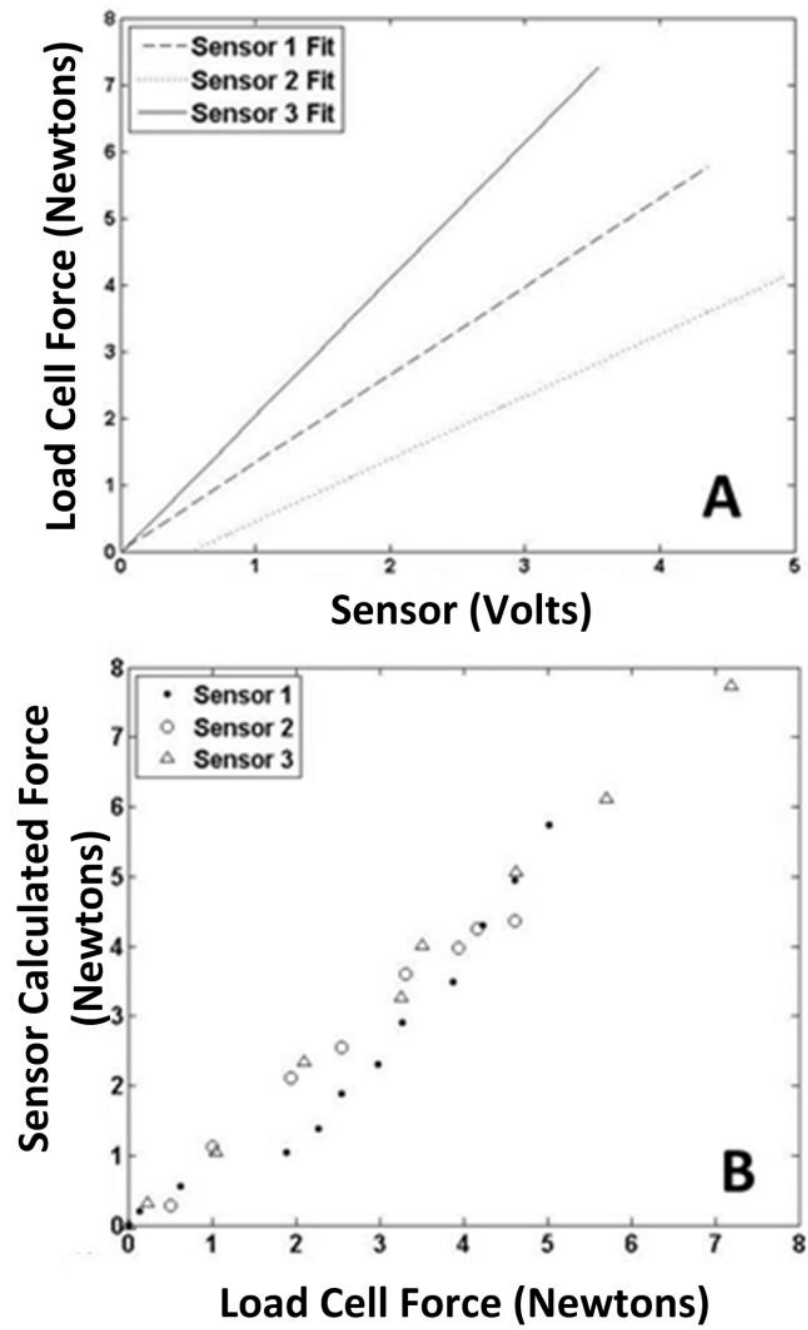

Figure 15: Force sensors fit linearly to the load cell produce three linear fitting equations with unique $m$ and $b$ (A). The calculated force from the force sensors is plotted against the true force (B) to show that all sensors produce similar responses to applied load when using the calibration fitting. 


\begin{tabular}{r|ccc}
\hline \multicolumn{4}{c}{ Force Sensor Fitting Parameters } \\
\hline Sensor & $m$ & $b$ & $R^{2}$ \\
\hline 1 & 2.0601 & 0.0314 & 0.9968 \\
2 & 1.3244 & 0 & 0.9049 \\
3 & 0.9385 & 0.4944 & 0.9833
\end{tabular}

Table 2: Fitting parameters for each sensor

15A). The values of parameters $m$ and $b$ were unique for each sensor and were such that each linear fit resulted in an $R^{2}>0.9$ [Table 2]. A testing set of data was gathered from the same sensors and estimated force was calculated from the parameters obtained in the fitting procedures (Figure 15B). The root mean square error values for Sensors 1,2 , and 3 were $0.76,0.05$, and 0.74 , respectively.

\section{Study 2: Biphasic Current Pulse Amplitude Adjustability Demonstration through $\alpha$}

Observed biphasic amplitude ranges from \pm 0.2 to $\pm 15 \mathrm{~V}$ for $\alpha$ value 1 and 255 , respectively (Figure 16). Note the different voltage range between Fig. 16 A and $\mathrm{B}$, as well as the biphasic pulse width of $0.1 \mathrm{msec}$. The signal has no overshoot or excess electrical noise. Each increasing increment of $\alpha$ result in an increase of
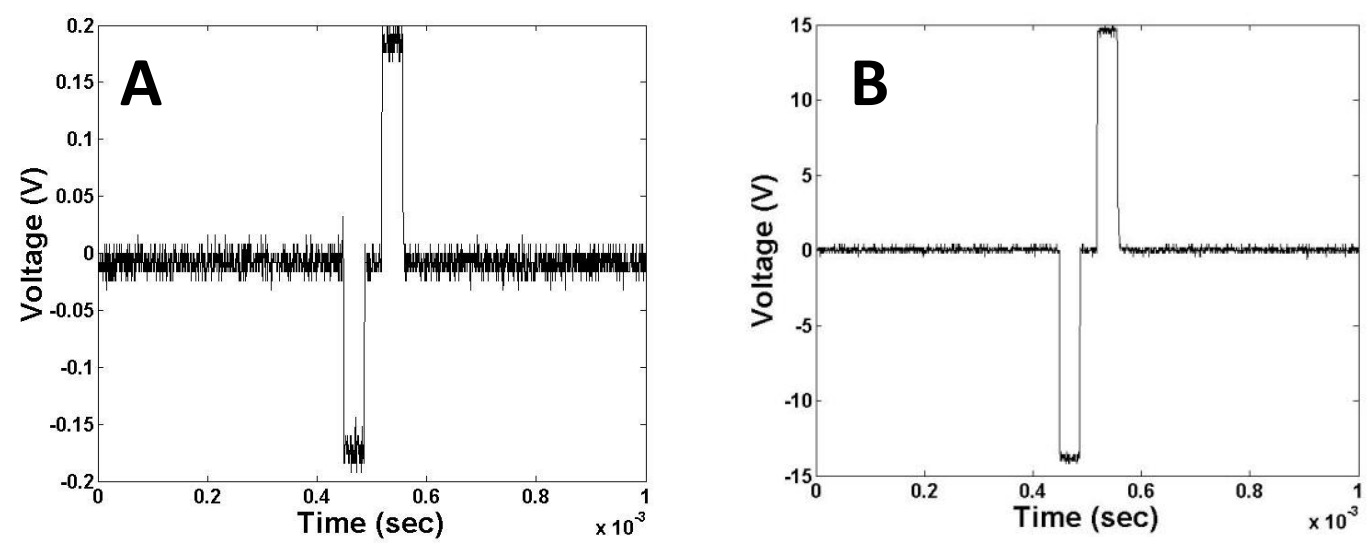

Figure 16: Single biphasic waveforms triggered with an oscilloscope range from \pm 0.2 (A) to $\pm 15 \mathrm{~V}$ (B) for $\alpha=1$ and 255 , respectively. Note the $x$-axis is displayed in seconds $E-3$, showing the waveform has a width of $0.1 \mathrm{msec}$, and the $y$-axis for $A$ and $B$ differ by an order of magnitude. 
amplitude by $\pm 0.0582 \mathrm{~V}$. The amount of current delivered to nerve is dependent on the type of electrode used. For an electrode providing $4 \mathrm{k} \Omega$ impedance, the current delivered from the biphasic pulse ranges between \pm 0.05 and $3.46 \mathrm{~mA}$ for \pm 0.2 and $15 \mathrm{~V}$, respectively. A complete mapping of $\alpha$ value to waveform voltage as well as waveform voltage to current is provided in Appendix E.

Study 3: Mimicking SAI Firing Rates in Response to Applied Force for the Nominal Case

Dynamic and static firing rates generated from the Tactile Modulation Platform were compared to those from the sural nerve in what we refer to here as the Nominal Case. Qualitatively, the increase in static firing rate with increasing static force is seen in Figure 17. In Figure 18, the mean predicted dynamic firing rate with a $k_{d}$ value of $5.7 e^{-2}(11.2,28.5,42.9$ spikes/sec $)$ was compared to the observed firing rate $(13.0,30.3,52.7$ spikes/sec) over increasing applied load (1.5,
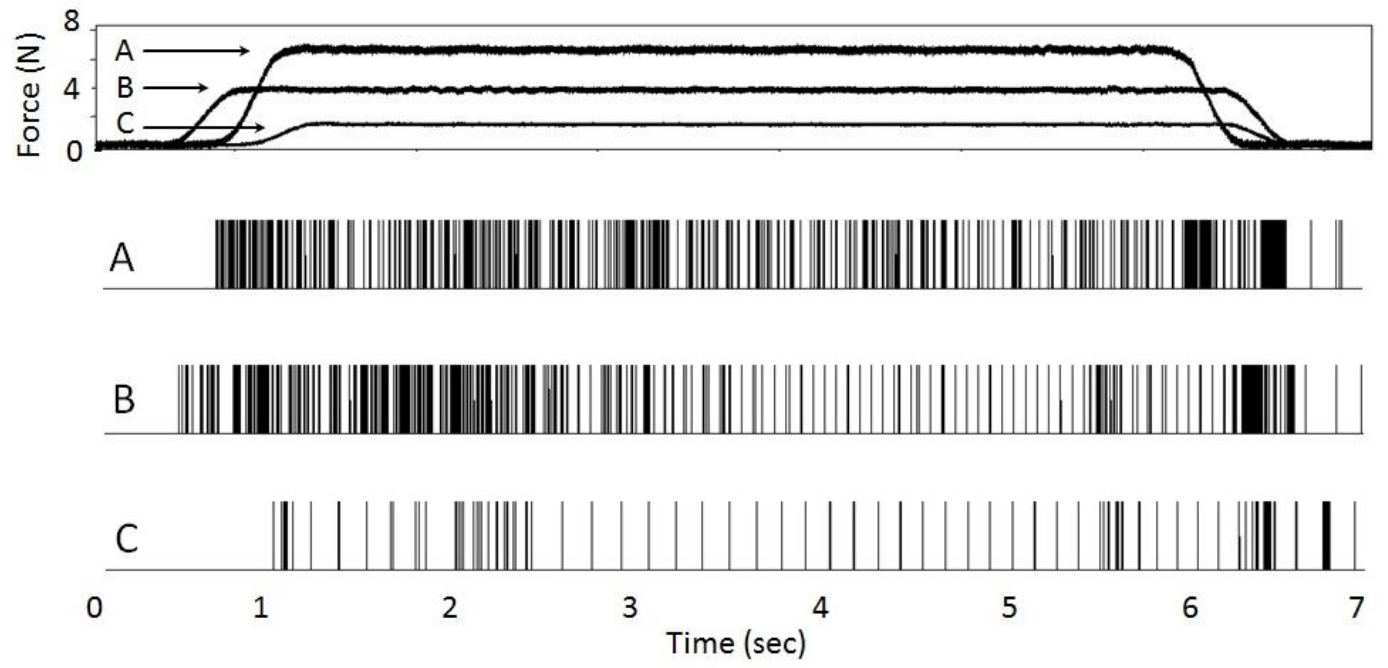

Figure 17: Tactile modulation platform current pulse timings in response to three levels of applied force $(1.5,4,7.5 \mathrm{~N} ; \mathrm{A}, \mathrm{B}, \mathrm{C})$. The calculated firing rates increase with increasing magnitude of applied force. 


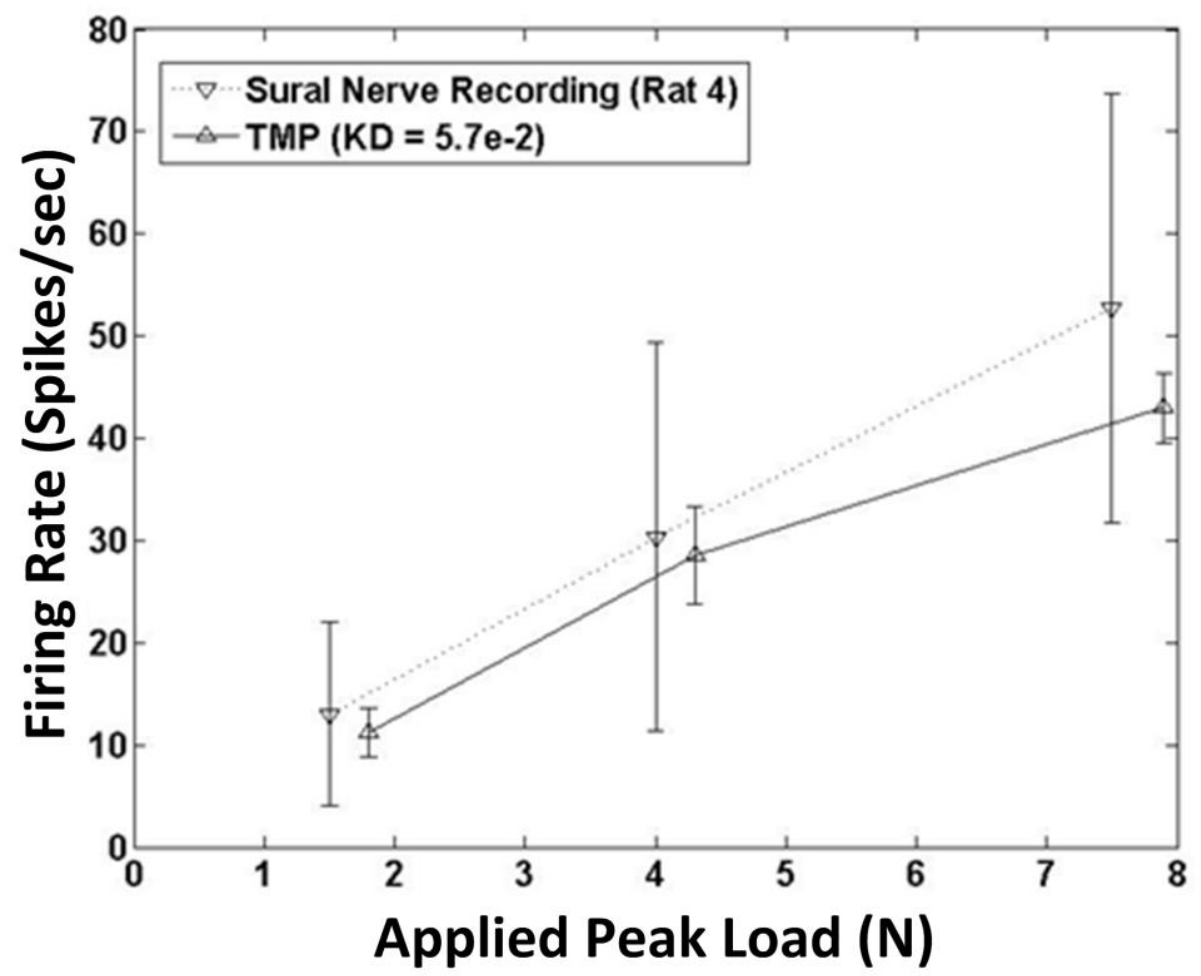

Figure 18: Comparison of dynamic firing rate means (error bars = SD) of the calculated (black) vs. observed (gray).

4, 7.5 N). Paired two-tail Student's t test comparisons of dynamic means across increasing applied peak loads suggest at a significance level of 0.05 , the means were not significantly different $(\mathrm{p}$ values $=0.9,0.8,0.05)$, except for the applied peak load of $7.5 \mathrm{~N}$, where there was a weak argument against the null hypothesis (there is no significant difference between the calculated and observed means).

Across increasing loads, the predicted static firing rate with a $k_{s}$ value of $4.2 e^{-6}$ $(7.5,14.5,30.1$ spikes/sec) were compared to the observed static firing rate $(5.0$, 12.9, 41.6 spikes/sec). Similarly, a paired two-tail Student's t-test comparisons of static means across increasing applied loads suggest at a significance level 0.01, we cannot reject the null hypothesis ( $\mathrm{p}$ values $=0.023,0.24,0.025$ ) that the calculated and observed means are significantly different (Figure 19). 


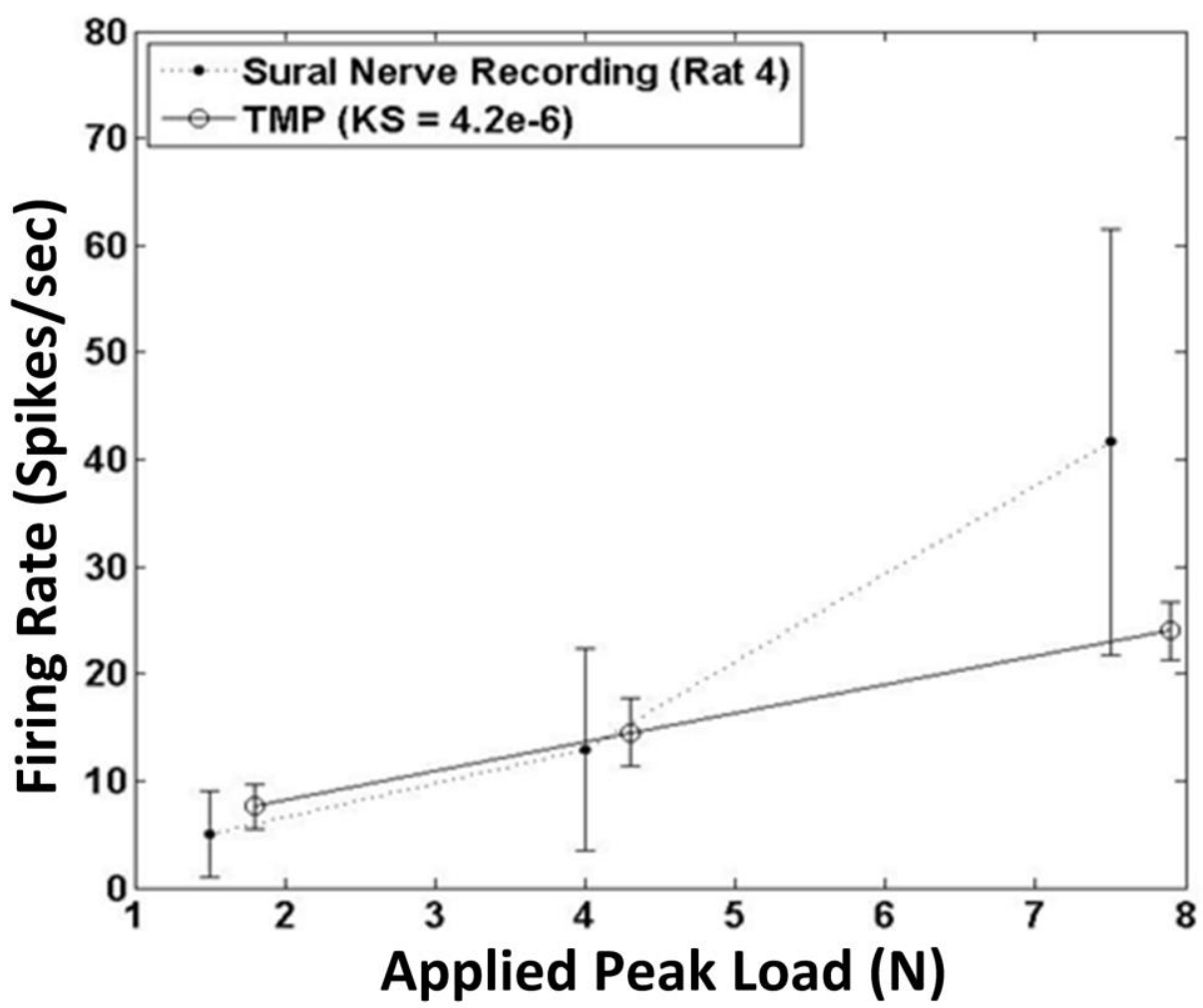

Figure 19: Comparison of static firing rate means (error bars $=\mathrm{SD}$ ) of calculated vs. observed (gray).

Study 4: Varying $\mathbf{k}_{\mathrm{s}}$ to Mimic Several Cases of Firing Rate Variability Observed in 3 Rats

The values of $k_{s}$ were varied for each of three rat cases $(9.7 \mathrm{E}-2,1.5 \mathrm{E}-4,4.2 \mathrm{E}-5$;

Rat $1,2,3)$ to achieve three separate discrimination sensitivity functions. For the silicone-force sensor set up used in these experiments, the lowest level of detectable at the force sensor was an applied load of $1.5 \mathrm{~N}$ at the silicone. It is for this reason that we cannot compare paired calculated firing rates to observed in the Rat 1 case, as each applied load is observed to be less than $1.5 \mathrm{~N}$. However, Figure 20 shows that for $k_{s}=9.7 e^{-2}$, the calculated firing rate at an applied peak load of $1.73 \mathrm{~N}$ is within the projected data. 


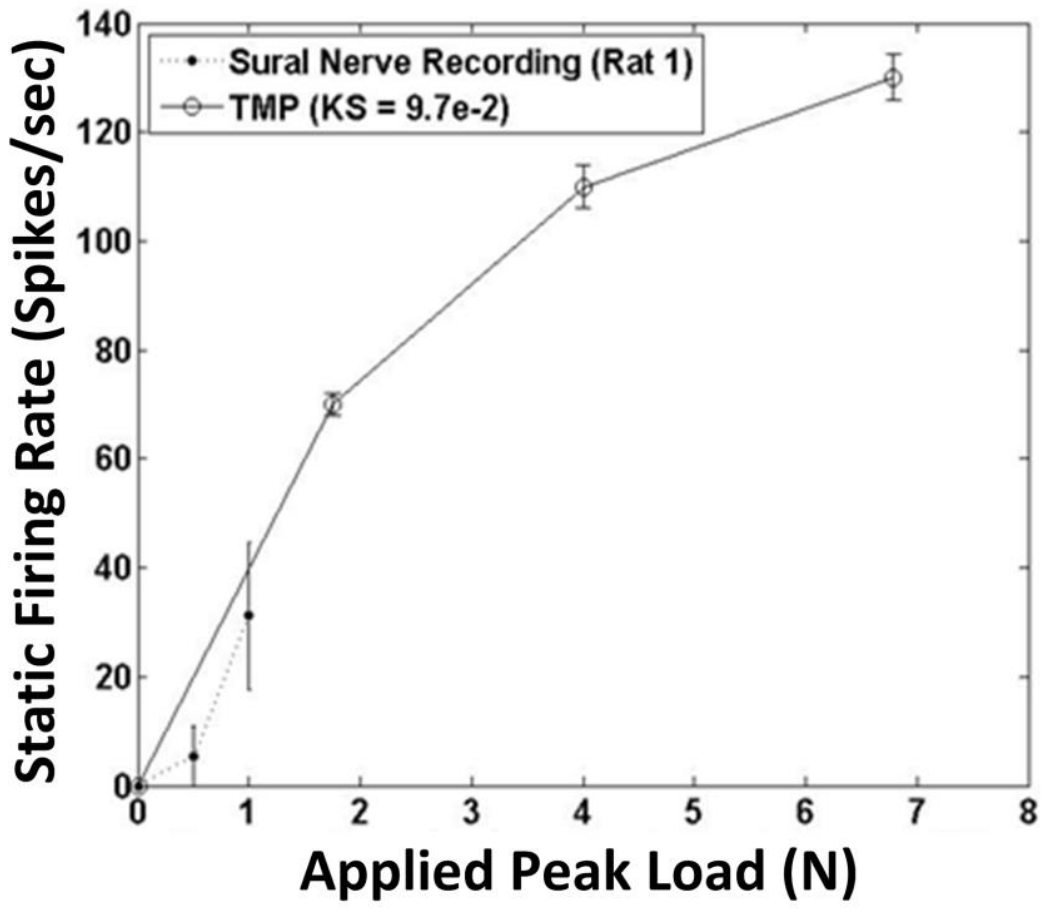

Figure 20: Calculated static firing rates (black solid) plotted with observed firing rates in Rat 1 (grey dotted).

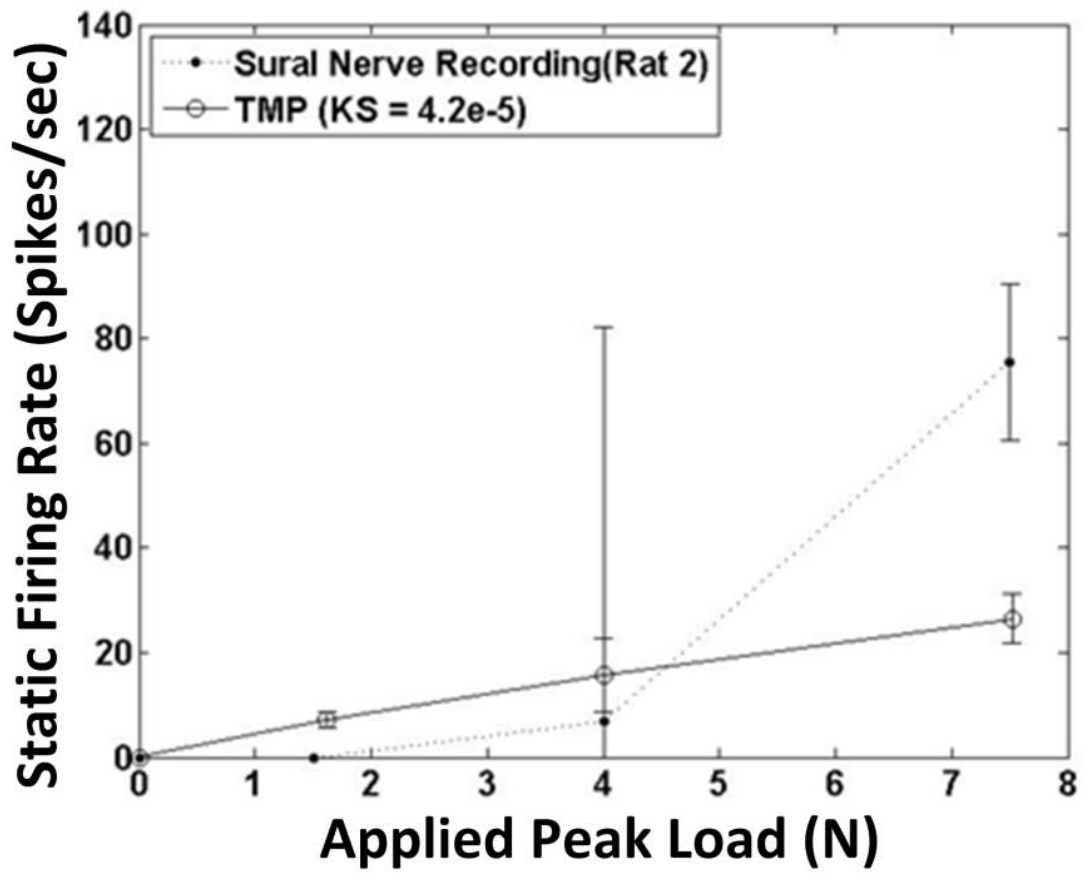

Figure 21: Calculated static firing rate (black solid) plotted with observed firing rates from Rat 2 (grey dotted) 


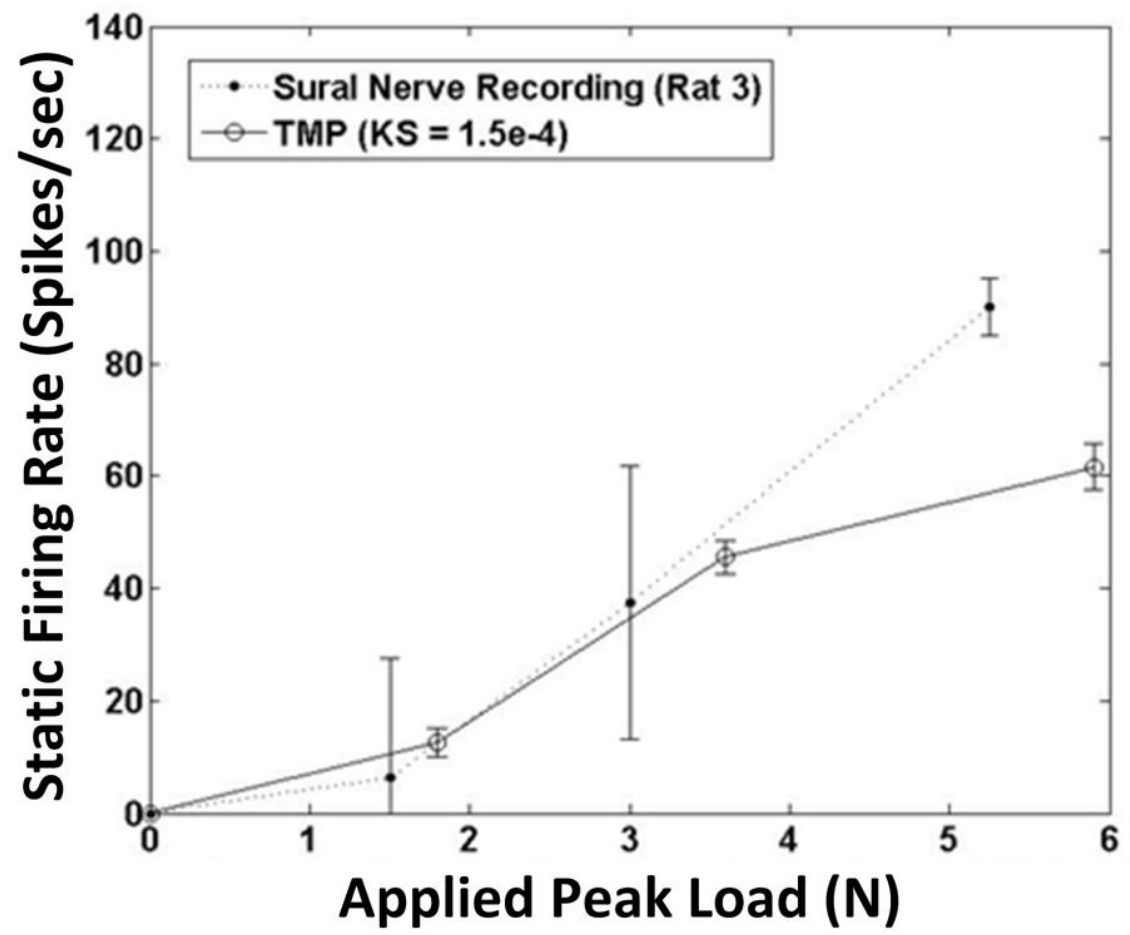

Figure 22: Calculated static firing rates (black solid) plotted with observed firing rates in Rat 3 (grey dotted).

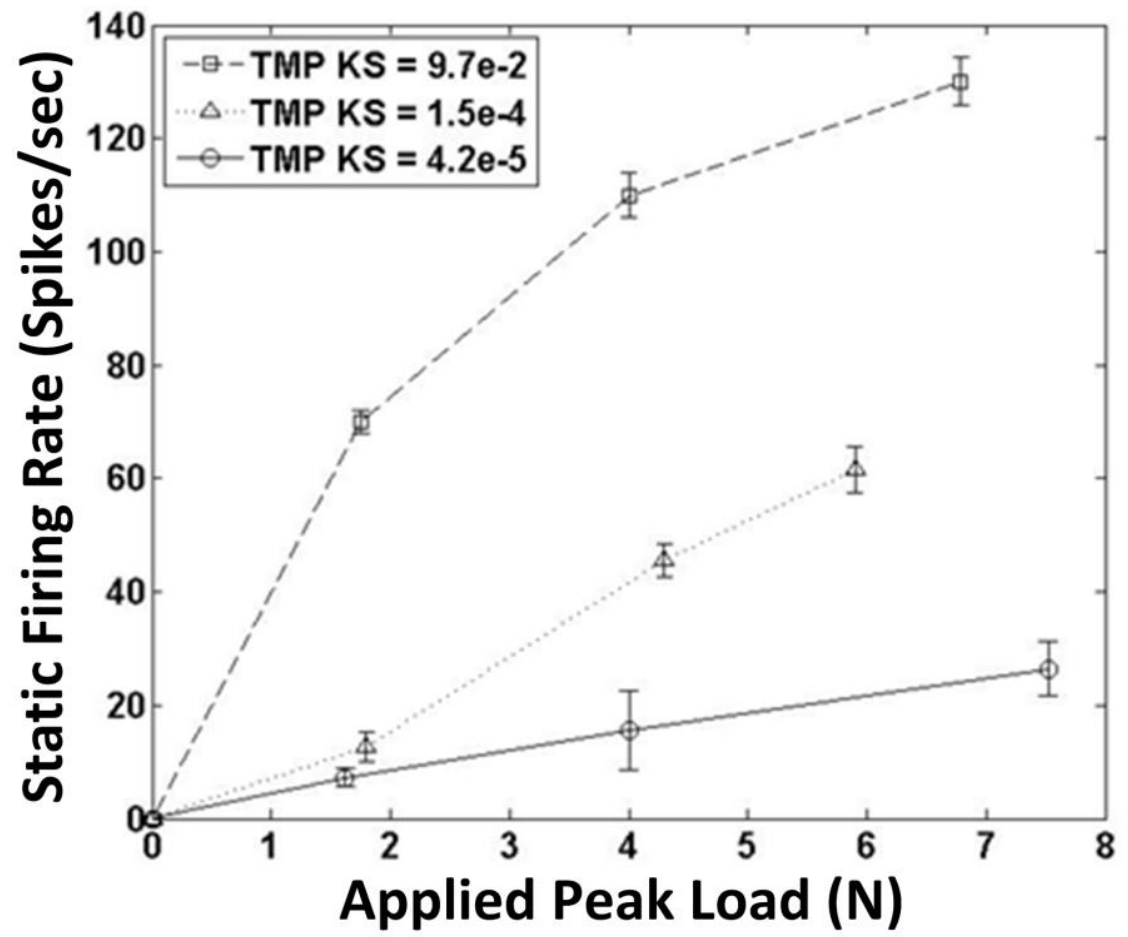

Figure 23: Calculated static firing rates from the Tactile Modulation Platform. Fach line represents a single sensitivity discrimination case, where rate of increasing firing rate becomes steeper for increasing values of Ks. Grey labels for each function represent the observed data (which rat) each aimed to fit. 
In contrast, decreasing the $k_{s}$ value to $4.2 e^{-5}$ produces a sensitivity discrimination function with the lowest firing rates observed in rats (Rat 2, Figure 21). To achieve the moderate sensitivity discrimination function, setting $k_{s}$ to $1.5 e^{-4}$ produces firing rates similar to that of Rat 3 (Figure 22). All three sensitivity discrimination functions are plotted in Figure 23. 


\subsection{Part 2: Calibration Procedures and User Interface Design}

This effort focuses on the design of three procedures for user calibration of parameters and a preliminary design of a graphical user interface to facilitate performing the procedures and afford ease of use, which are integrated into the Signal Transformation Apparatus to create the entire Engineered Tactile Modulation Platform (Figure 24).

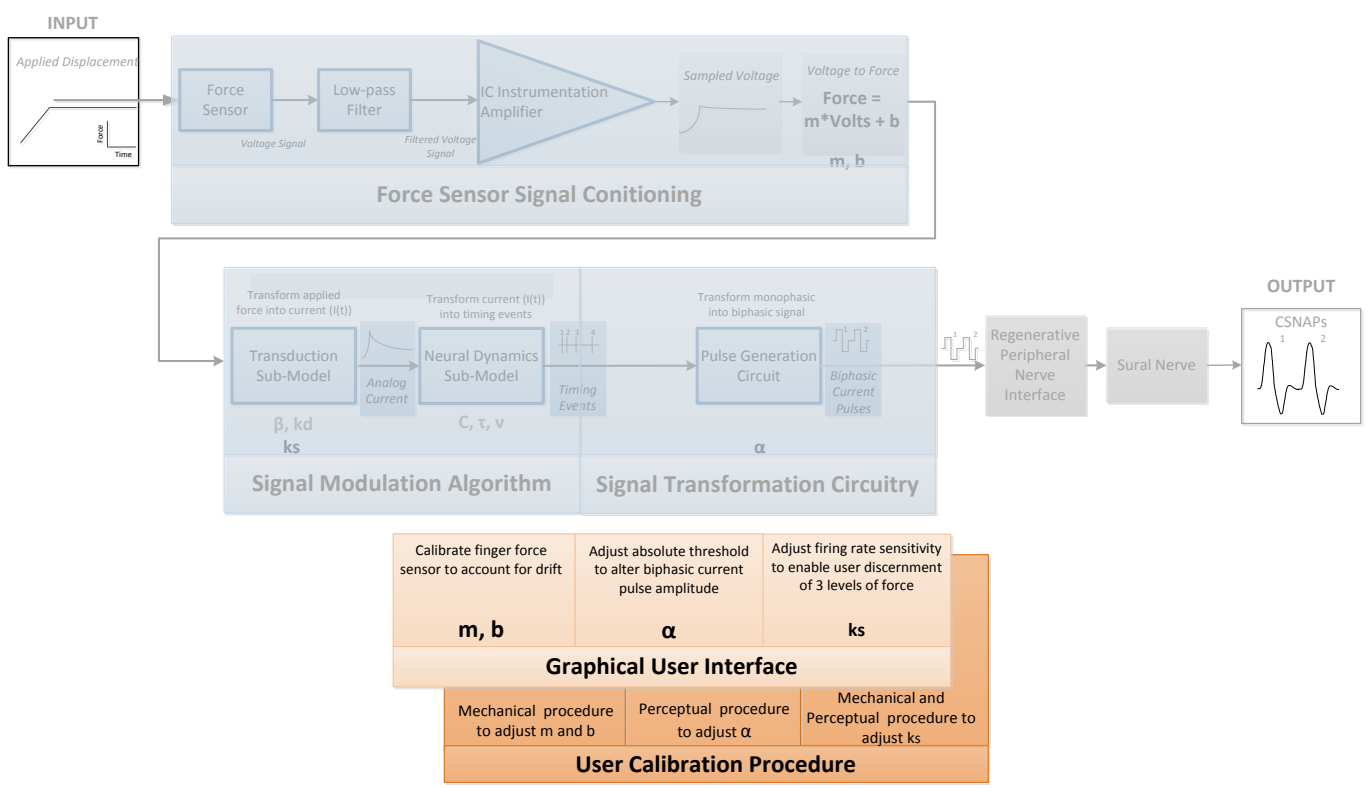

Figure 24: The Graphical User Interface along with User Calibration Procedures alter platform parameters

Consideration of how the user will interact with the platform in practice is central to the design of calibration procedures. These demonstrate the GUI's ability to perform calibration tasks for the force sensor, nerve absolute detection threshold, and the nerve firing rate sensitivity. Simple tasks imbed logic and psychophysical experiments the user proceeds through in order to calibrate parameter values. 


\subsubsection{FORCE SENSOR CALIBRATION}

A user interface enables the force sensor calibration procedure to be completed to account for sensor variability. Additional equipment beyond the prostheses and mobile GUI needed include a flat surface, such as a table, and load cell to serve as a comparison standard. The calibration apparatus is detailed in Figure 25 and shows how the user engages with the load cell and user interface. The user will be instructed through the procedure, wherein the sensor will be depressed by the user into the load cell over a range of forces (1-4 N). A graphic displayed on the user interface provides feedback to the user as to what the target force (Figure 25, dotted line) and current finger force sensor reading are (Figure 25, purple bar). The process below is repeated until stopping criterion is met, defined as when $R^{2}>$ 0.9. Appendix $\mathrm{F}$ provides a progression of the procedure through a first version GUI.

\section{Force Sensor Calibration Procedure}

1) Press onto scale with finger until $1 N$ target is reached, please hold for 5 seconds

2) Remove finger from scale

3) Press onto scale with finger until $0.5 \mathrm{~N}$ target is reached, please hold for 5 seconds

4) Remove finger from scale

5) Press onto scale with finger until $0.75 N$ target is reached, please hold for 5 seconds

6) Repeat $n$ times until stopping criterion are met 


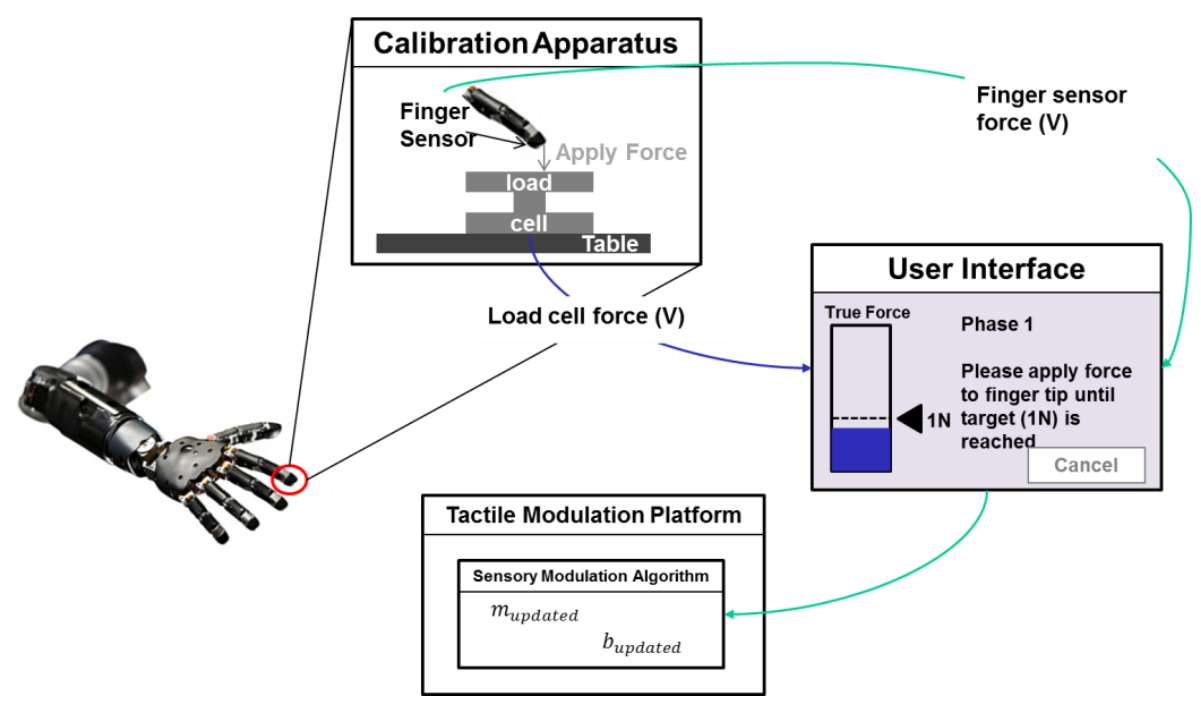

Figure 25: Finger Force Sensor Calibration Interface and Procedure

\subsubsection{NERVE ABSOLUTE THRESHOLD CALIBRATION}

Recall the desire to limit the amount of current delivered to the nerve as discussed in Section 2, where $\alpha$ determines the amplitude of current pulses (Signal Transformation Circuitry), and the neurophysiological limitations surrounding electrical stimulation such that recording electrodes are infeasible.

For $\alpha$ to be calibrated, the human user must provide feedback. The user interface provides the medium for the human to follow a process to achieve this (Figure 26). When the absolute nerve threshold button is selected from the nominal screen, the user interface begins the procedure shown below. First, a pulse amplitude of $\alpha-3 \mathrm{~h}$, where $\mathrm{h}$ is the step size, is sent to the nerve. For each trial (Figure 27A), a pulse train (Figure 27B) is delivered with specified $\alpha$. The procedure is designed as a forced-choice staircase psychophysical experiment, such that when three opposite transitions have been repeated in a row, the 


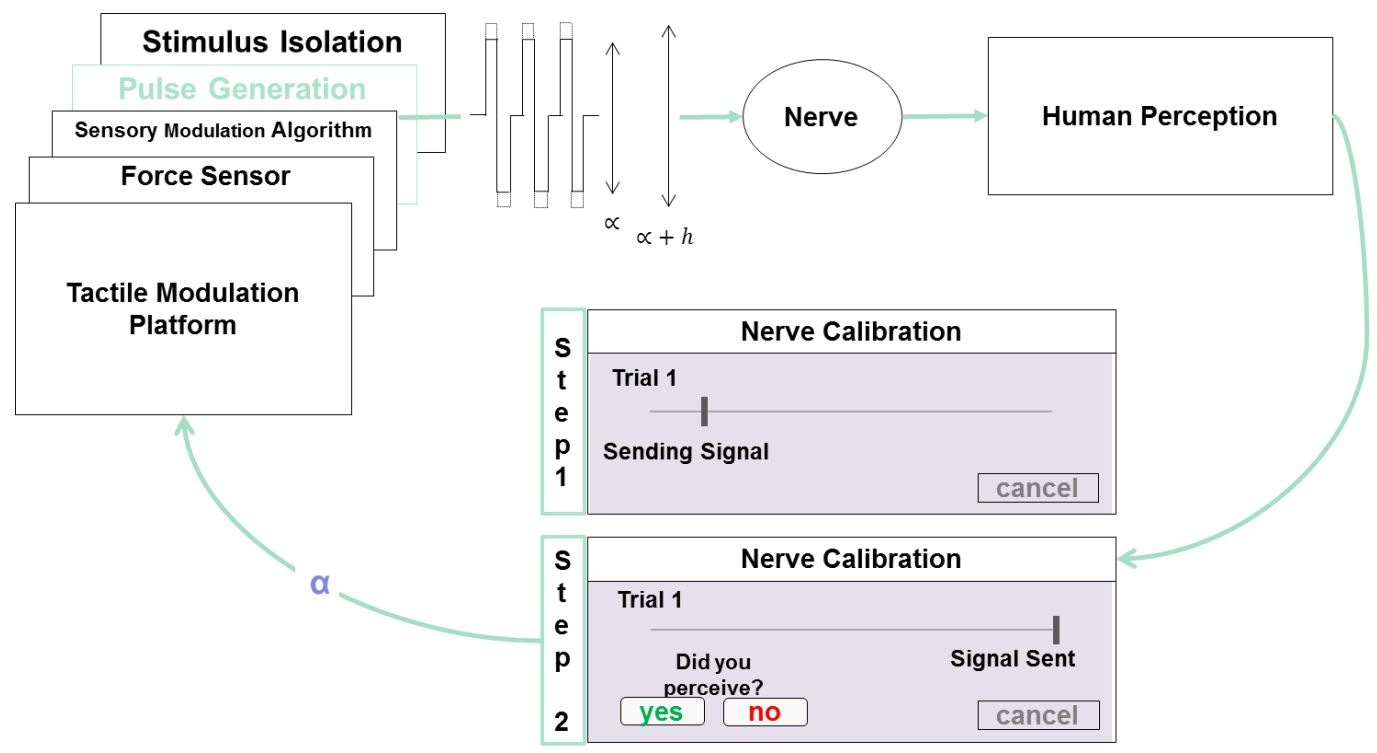

Figure 26: Absolute Nerve Threshold Calibration Procedure System

stopping criteria are satisfied and the final parameter for $\alpha$ is retained within the signal modulation platform. The only information displayed to the user is when a signal is being sent and when the trial has ended to prevent bias. At this point, the user is asked if he/she perceived the signal. A step-by-step scenario of this procedure is shown in Appendix G.

\section{Absolute Nerve Threshold Calibration Procedure:}

1) Send a charge balanced current pulse train with $\propto_{\text {test }}=\propto_{\text {now }}-$ 3 (slightly lower than the current amplitude setting) to the nerve

2) Ask the patient if he/she perceived the signal

3) If the signal is perceived, set $\propto_{\text {test }}=\propto_{\text {now }}-h$

4) If the signal is not perceived, set $\propto_{\text {test }}=\propto_{\text {now }}+h$

5) Repeat process until stopping criterion is reached 


\section{Forced Choice Staircase Method (Pulse Amplitude vs. Trials)}

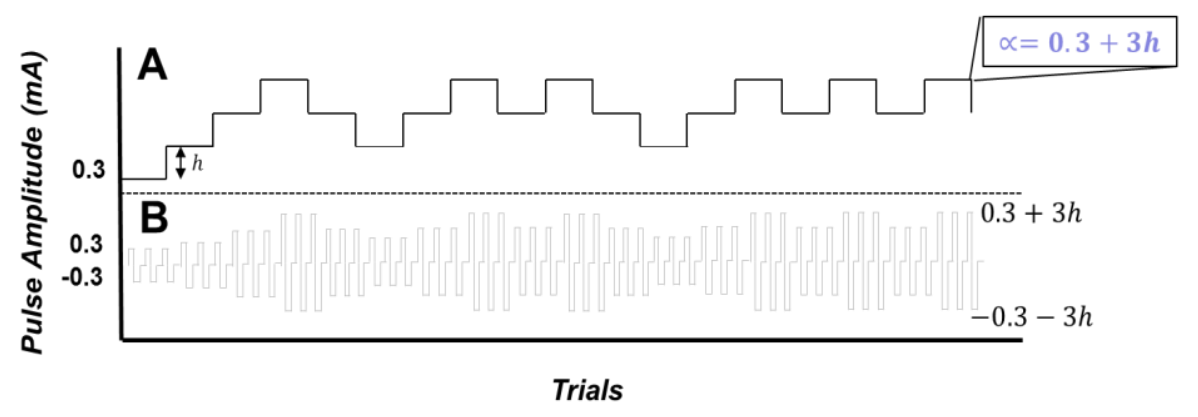

Figure 27: Psychophysical forced choice staircase method over many trials.

\subsubsection{NERVE DISCRIMINATION SENSITIVITY CALIBRATION}

The procedure to calibrate the nerve over a range of applied force is initiated when the orange "nerve sensitivity" button is selected from the nominal screen. The process is shown below:

\section{Nerve Firing Rate Sensitivity Calibration Procedure:}

1) Ask user to apply force until the user interface indicates he/she is applying the correct amount and hold this amount for 10 seconds. For the first trial, the target is $1 \mathrm{~N}$, but to prevent bias, this is not revealed to the user. During this process, the tactile modulation platform sends a charge balanced current pulse train with $\alpha$ and $k_{s}$, where $\alpha$ is calibrated in the Nerve Absolute Detection Threshold Calibration Procedure (shown above). This is considered the base case for comparison.

2) Ask the user to apply force until the user interface indicates he/she is applying the correct amount and hold the force for 10 seconds. In the second trial, the target is $2 \mathrm{~N}$, and again this is concealed from the user. The tactile modulation platform will now send a signal with $k_{s}$ _updated $=k_{s}+h . K_{s}$ is isolated in this experiment because the user is in static hold phase due to the constant applied force. 
3) The user interface then asks the user if a difference was perceived between the two signals

- If the answer is yes, proceed to step 4.

- If the answer is no, the process is started again with step 2 incrementing by $2 * h$. Unless there is a perceivable difference, step 2 is incremented by an additional level $h$ until the answer in step 3 is yes

4) The signal from step 2 is set to $k_{s}-h$, and steps 1 and 2 are repeated:

- The user interface then asks the user if a difference was perceived between the two signals

* If the answer is no, the process is repeated from step 1 until stopping criteria of 3 oscillatory responses are received.

* If the answer is yes, the process is repeated from step 1 until 2 additional oscillatory responses are received.

5) Repeat process until stopping criterion is reached (3 oscillatory responses)

This process is repeated until the stopping criteria are met. An example of a multiple trial experiment is shown in Figure 28, where A depicts the trial as a staircase and B shows corresponding pulse trains for each trial in A.

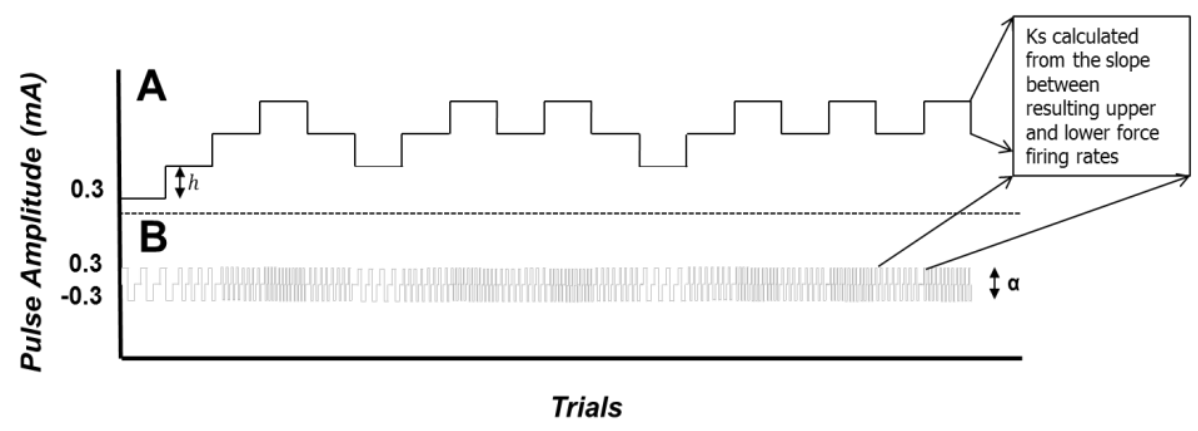

Figure 28: Forced Choice Staircase Method ( $k_{s}$ vs. Trials) 


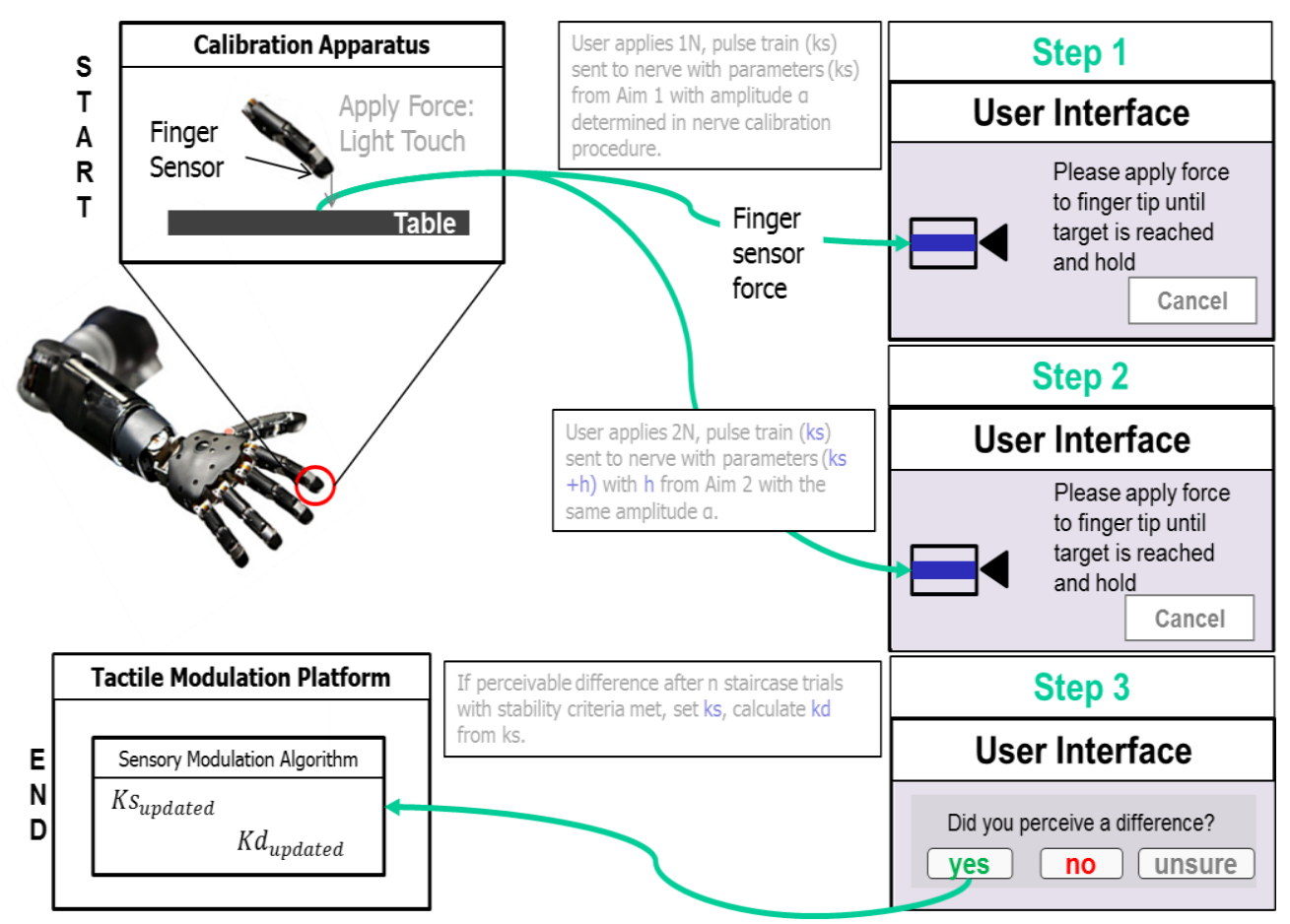

Figure 29: Firing Rate Sensitivity Calibration System and Procedure

The system and user interface design for each step in the process is shown in Figure 29. Notice the only information revealed to the user is a target force void of numerical values to prevent bias. If the user does not perceived a difference between the two forces, then the value of $k_{s}$ is increased by h. Also, note that this procedure isolates $k_{s}$ because the user is not changing the applied force, which eliminates the term $k_{d}$ (change in force is 0) (Eqn 2). Appendix $\mathrm{H}$ contains step by step progression through the process within the GUI.

\subsubsection{PRELIMINARY USER INTERFACE DESIGN}

The preliminary user interface is designed for a mobile device with the capability to interface with the signal transformation apparatus via either a USB or wireless connection, allowing parameters to be calibrated without accessing the algorithm housed on the microcontroller. 


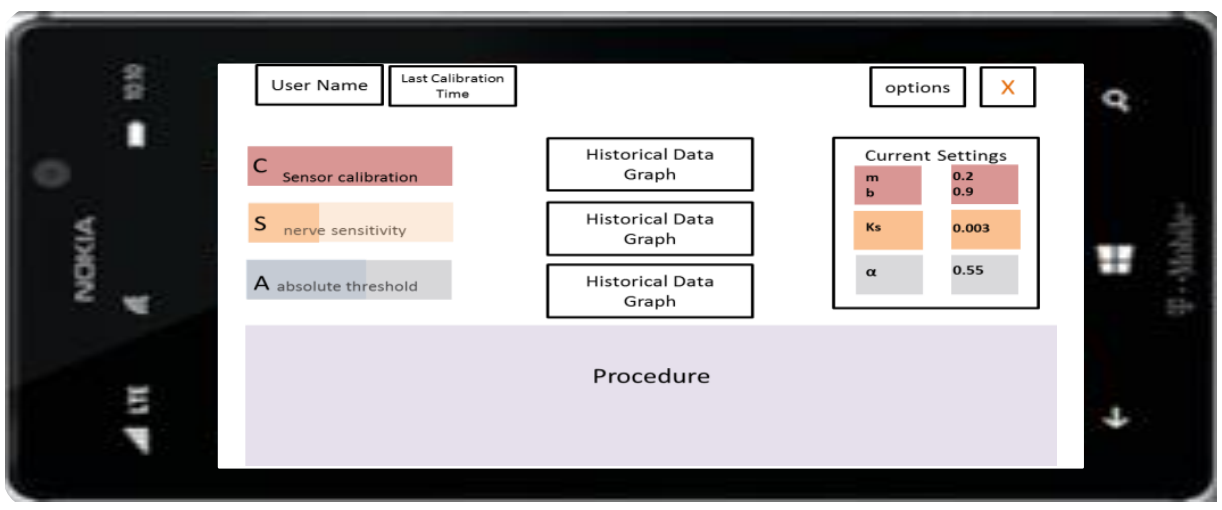

Figure 30: Design of Mobile Graphical User Interface

The nominal view of the user interface is shown in Figure 30. From this screen, the user can navigate to adjust the parameters by selecting icons on the left side of the display. These icons are shaded to show how the current setting relates to the upper and lower limits of the parameter; for example, an absolute threshold of 7.5 $\mathrm{V}$ is depicted to fall in between the upper and lower limits as the icon is half shaded. When an icon is selected, a drop down menu appears below the macro view of parameters. This allows the user to make changes to the selected parameter without navigating away from the nominal view, maintaining both macro and micro depths of information. Historical graphs allow the user to view how parameter settings are changing over time, while login information and options reside in an upper banner. 


\section{DISCUSSION}

This work provides a user-centered approach to the design of a mobile, tactile modulation platform, comprised of a signal transformation apparatus (leakyintegrate-and-fire based algorithm, signal transformation circuitry, and parameter calibration procedures), to not only mimic spiking rates in dynamic and hold phases of indentation stimuli, but to facilitate intuitive adjustment of the platform, accounting for three phenomenon: i) force sensor variability, ii) nerve absolute detection threshold, and iii) nerve discrimination sensitivity. The platform was validated first against a nominal case, and then three additional variation cases of dynamic and static firing rates observed in the rat. Adjustability of sensor gain parameters were evaluated among three force sensors, while the absolute detection parameter was systematically varied to provide a working range of biphasic signal amplitudes output from the signal transformation apparatus. Overall, the platform firing rate predictions fit well with those observed among rats and remaining parameters accounted for expected variability.

This work demonstrated predictions of dynamic and static hold firing rates for three levels of applied force that match those observed in the nominal rat sural nerve. As the applied force increased from $1.5 \mathrm{~N}, 4 \mathrm{~N}$, to $7.5 \mathrm{~N}$, the signal transformation apparatus predicted an increasing trend in dynamic firing rates $(11.2,28.5,42.9$ spikes/sec) and static firing rates $(7.5,14.5,30.1$ spikes/sec). These predictions fit favorably with those observed in the rat for both dynamic $(13.0,30.3,52.7$ spikes/sec) and static (5.0, 12.9, 41.6 spikes/sec) phases, with pvalues > 0.05 and 0.01 , respectively. 
For the first time, this work has demonstrated fitting static hold firing rates to those of three rats, each with a unique discrimination sensitivity function. Because static firing rates are known to increase with a linear trend [21], fitting the single parameter $k_{s}$ to cases with varying rates of increasing firing rates across increases in applied peak force resulted in favorable fits for rat cases 1, 2, and 3. Rat 1 provided the most sensitive discrimination function, where the average static firing rate was observed to increase from 9.2 to 36.9 spikes/sec over applied peak loads of 0.5 and $1 \mathrm{~N}$, respectively. Although the platform did not respond to applied loads less than $1.5 \mathrm{~N}$, the predicted firing rates followed a similar linear trend, where firing rates increased from 70, 100.2, to 129.8 spikes/sec across applied peak loads of 1.7, 4, $6.9 \mathrm{~N}$. To obtain a more sensitive sensor-substrate, future work may aim to position the force sensor in a shallower silicone-substrate. Rat 2 provided the least sensitive discrimination function, as the predicted static firing rates: 7.2, 15.6, and 26.5 spikes/sec, aligned with a linear fit average observed static firing rates: $0,6.7$, and 75.4 spikes/sec. It should be noted that there exists variation in neurophysiological data (standard deviation $\pm 0,75.1$, 50.3, respectively for rat 2). The objective in fitting this case was to provide a low sensitivity discrimination function that aligned with a linear fitting of the available rat 2 data.

In this work, we used a linear calibration procedure to fit force sensor outputs to known applied loads. Although the relationship between applied force and sensor output is known to be linear, this relationship does not necessarily hold when additional mediums, such as a silicone-substrate, intercede the reception of load to 
the sensor. In fact, silicone is a hyper-elastic material, and as such responds linearly to applied low forces and then becomes non-linear. This behavior is observed in the results of this thesis: linearly applied force at the substrate does not correspond to linearly received force at the embedded sensor (Figure 31).

The artifact from this relationship is the non-linear predicted firing rates output from the Signal Modulation Algorithm. Among all cases surveyed, a linear trend of predicted firing rates was observed in response to applied loads at the substrate between $1.5 \mathrm{~N}$ and $4 \mathrm{~N}$. However, the same trend does not continue for applied loads of $7.5 \mathrm{~N}$ and can be seen in both Study 3 and Study 4 results. Although the predicted firing rates still fall within the large standard deviations observed in rats, a more linear fit may be obtained if the silicone embedded force sensor was calibrated to the load cell. The calibration fitting would most likely be non-linear and take on more of a logarithmic or polynomial approximation.
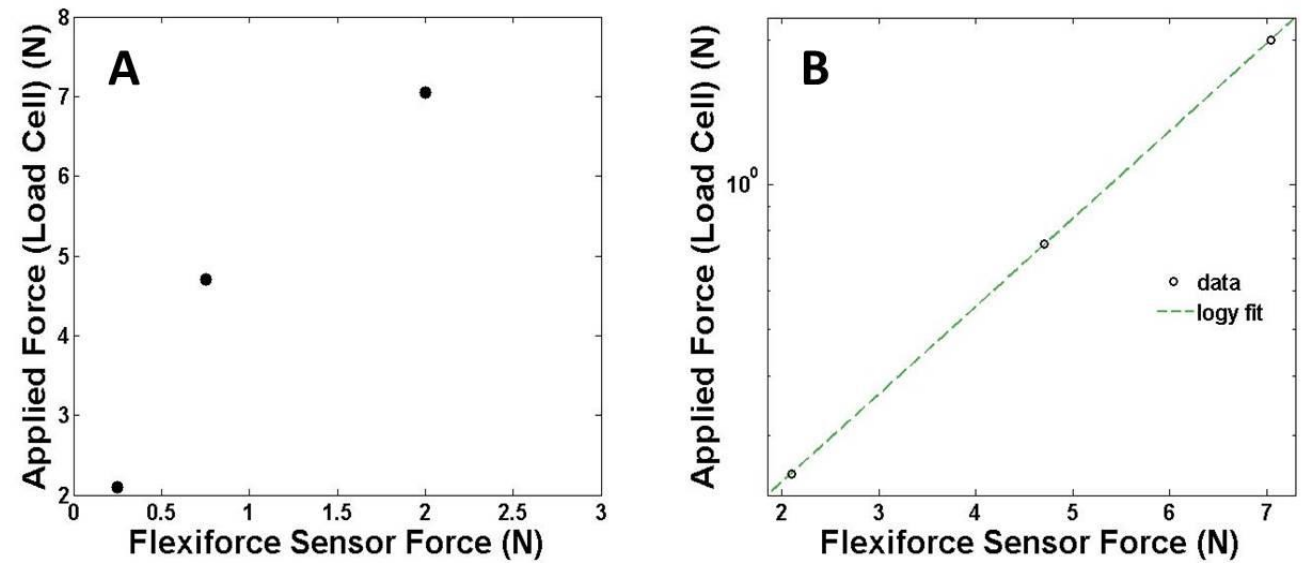

Figure 31: A linearly calibrated force sensor embedded within silicone produces a non-linear trend of applied load at the substrate to recorded force at the sensor (A). A logarithmic fitting of this data produces a goodness of fit equal to 1 (B). Note the $y$-axis scale in $B$ is logarithmic. 
One main contribution of this thesis is the ability of the platform to account for intrinsic variability of both hardware and neurophysiological mechanisms. Here, we evaluate three sensors for variability of responses to applied force, but do not directly investigate individual sensor drift over time due to time constraints. Similarly, we take into account the absolute detection threshold variability between rats, but do not observe how nerves respond to electrical stimulation over time. We can hypothesize both how an individual sensor or nerve may behave over time from observed variability between subjects, but for future work, gathering this data will provide more concise direction for the calibration procedures.

The identification of: 1) the sources and, 2) parameters to account for variability while concurrently designing a set of user-centered calibration procedures is essential to the applicability of this platform in the future. Not only do users with limited mathematical and programming background need to easily calibrate the system, but the parameters that psychophysical experiments can account for are limited. For example, the static gain of the transduction function was identified for calibration, but the dynamic gain was not. This is because the dynamically applied force is difficult to accurately replicate from the user perspective and nearly impossible to quantify with a scale.

Without using physical experiments to evaluate parameters within the Signal Modulation Algorithm, discovering emergent features, such as sensor gain and absolute detection parameters effect on firing rate and waveform outputs, would have been difficult. Now that these parameters have been identified and 
evaluated, this work can be applied without software. An approach using resistive capacitive (RC) circuitry to model the leaky-integrate-and-fire neuronal behavior would expand the number of sensors simultaneously inputting the platform, reduce the amount of power required to power the platform, as well as miniaturize the entire device. 


\section{REFERENCES}

[1] E. Biddiss, D. Beaton, and T. Chau, "Consumer design priorities for upper limb prosthetics," Disabil. Rehabil. Assist. Technol., vol. 2, pp. 346-357, Nov. 2007.

[2] D. Moniz. (2005, 10 05). Arm amputees rely on old devices. USA TODAY, 05Oct-2005. [Online].

Available: http:/usatoday30.usatoday.com/news/nation/2005-10-05-arm-amputees_x.htm. [3] Lewis, S.; Russold, M.F.; Dietl, H.; Kaniusas, E.; , "User demands for sensory feedback in upper extremity prostheses," Medical Measurements and Applications Proceedings (MeMeA), 2012 IEEE International Symposium on, vol., no., pp.1-4, $18-$ 19 May 2012 http://ieeexplore.ieee.org/stamp/stamp.jsp?tp=\&arnumber=6226669\&isnumber $=6$ 226618

[4] Stieglitz, T., "Considerations on Surface and Structural Biocompatibility as Prerequisite for Long-Term Stability of Nerual Prostheses," J. Neurosci and Nanotech, vol 4, pp. 496-503(8), 2004.

[5] J.E. O’Doherty, M.A. Lebedev, P.J. Ifft, K.Z. Zhuang, S. Shokur, H. Bleuler, M.A.L. Nicolelis,, "Active tactile exploration using a brain machine-brain interface," Nature, vol. 479, no. 7372, pp. 228-231, Oct. 2011.

[6] P.D. Marasco, K. Kim, J.E. Colgate, M.A. Peshkin, T.A. Kuiken "Robotic touch shifts perception of embodiment to a prosthesis in targeted reinnervation amputees," Brain. vol.134, pp. 747-758, Mar. 2011.

[7] C. Cipriani, M. Controzzi, M.C, Carrozza, "The SmartHand transradial prosthesis," Journal of NeuroEngineering and Rehabilitation, vol. 8, no. 29, May. 2011.

[8] S. Micera, P. Rossini, J. Rigosa, L. Citi, J. Carpaneto, S. Raspopovic, M. Tombini, C. Cipriani, G. Assenza, M. Carrozza, K.P. Hoffmann, K. Yoshida, X. Navarro, P. Dario, "Decoding of grasping information from neural signals recorded using peripheral intrafascicular interfaces," Journal of NeuroEngineering and Rehabilitation, vol. 8, no. 53, Sep. 2011.

[9] Dhillon, G.S., Horch, K.W., "Direct neural sensory feedback and control of a prosthetic arm," IEEE Transactions on Neural Systems and Rehabilitation Engineering, vol 13, pp. 468-472, 2005.

[10]Yoshida, K.; Horch, K., "Selective stimulation of peripheral nerve fibers using dual intrafascicular electrodes," Biomedical Engineering, IEEE Transactions on, vol.40, no.5, pp.492,494, May 1993 doi: $10.1109 / 10.243412$

[11] Lesniak, D.R. and Gerling G.J. Predicting SA-I mechanoreceptor spike times with a skin-neuron model, Mathematical Biosciences, 220, 15-23 2009

[12] Bensmaia S.J., Kim S.S., Sripati A.P. \& Vogelstein RJ (2008). Conveying tactile feedback using a model of mechanotransduction, Proceedings of IEEE Biomedical Circuits and Systems Conference, Baltimore, MD 
[12] Freeman, A.W., Johnson, K.O., "A model accounting for effects of vibratory amplitude on responses of cutaneous mechanoreceptors in macaque monkey," $J$. of Physiol, pp. 43-64, 1982.

[13] Johnson, K.O.,"The roles and functions of cutaneous mechanoreceptors," Current Opinion in Neurobiology," vol. 17, pp. 481-496, 2001.

[14] Johnson, K.O., Yoshioka, T., Vega-Bermudez, F., "Tactile functions of mechanoreceptive afferents innervating the hand," J. of Clinical Neurophysio., vol. 17 , pp. 539-558, 2000.

[15] Phillips, J.R., Johnson, K.O., "Tactile spatial resolution. III. A continuum mechanics model of skin predicting mechanoreceptor responses to bars, edges, and gratings," J. of Neurophysio., vol. 46, pp. 1204-1225, 1981.

[16] Kim, E.K., Sugg, K.B., Langhals, N.B., Lightbody, S.M., Baltrusaitis, M.E., Urbanchek, M.G., Cederna, P.S., Gerling, G.J., An Engineered Tactile Afferent Modulation Platform to Elicit Compound Sensory Nerve Action Potentials in Response to Force Magnitude, Proceedings of the IEEE World Haptics Conference 2013, The 5th Joint Eurohaptics Conference and IEEE Haptics Symposium, April 14-17, 2013, Daejeon, South Korea, pp. 241-230.

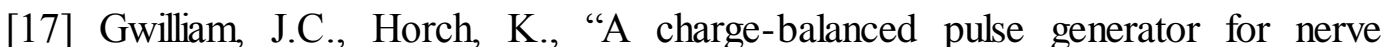
stimulation applications," J. of Neurosci Methods, vol. 168, pp.146-50, 2008.

[18] Land, B.R., Johnson, B.R., Wyttenbach, R.A., Hoy, R.R., "Tools for Physiology Labs: Inexpensive Equiptment for Physiological Stimulation," $J$. of Undergrad. Neurosci. Edu., vol 3, pp. A30-A35, 2004.

[19] Kim, E.K. Integrating Sensors with a Neural Model to Mimic SAI Tactile Spiking Response for Ramp and Hold Stimuli (Master's Thesis). University of Virginia, Charlottesville, VA.

[20] Tekscan. "Flexiforce Sensors User Manual." Tekscan. Tekscan, Inc., 29 Dec. 2010. Web. 22 Mar. 2014.

[21] Mountcastle, P.D., Schultz, A.E., \& Kornhuber, H.H. "The neural transmission of mechanical stimuli delivered to the monkey's hand," Ciba Foundation Symposium. Touch, Heat, and Pain. pp.325-351. 1966. 


\section{APPENDIX A}

\section{Silicone-Elastomer Force Sensor Substrate Procedure:}

Place plastic on a level surface, such as a table. Silicone materials were ordered from BJB Enterprises. Additional materials needed include disposable mixing containers and a mixing stick.

\section{Pouring the $1^{\text {st }}$ layer:}

1) Prepare PVC plastic cap mold by cleaning and applying a thin layer of non-stick spray ("Rapid Release", Stoner) along the bottom and inside walls and set aside

2) Pour $80 \mathrm{~g}$ of TC5005-A into a clean mixing container

3) Pour $12 \mathrm{~g}$ of TC5005-B into the same container. Mix for 5 minutes

4) Let stand for 25 minutes

5) Pour $0.88 \mathrm{~g}$ of TC5005-C into the container. Mix for 5 minutes.

6) Let stand for 10 minutes.

7) Place container with mixed material into a vacuum chamber for no more than 5 minutes

8) Remove from the chamber and immediately pour into PVC plastic cap to desired $9 \mathrm{~mm}$ level.

9) Wait 12 hours.

\section{Placing the sensor:}

1) Place sensor centrally on the silicone. Sensor should stick to tacky surface.

2) Wait 12 hours.

\section{Pouring the $2^{\text {nd }}$ layer:}

1) Pour $80 \mathrm{~g}$ of TC5005-A into a clean mixing container

2) Pour $12 \mathrm{~g}$ of TC5005-B into the same container. Mix for 5 minutes

3) Let stand for 25 minutes

4) Pour $0.88 \mathrm{~g}$ of TC5005-C into the container. Mix for 5 minutes.

5) Let stand for 10 minutes.

6) Place container with mixed material into a vacuum chamber for no more than 5 minutes

7) Remove from the chamber and immediately pour into PVC plastic cap to desired $9 \mathrm{~mm}$ level.

8) Wait 24 hours.

\section{Removing sensor-substrate:}

1) Carefully pry sensor-substrate from mold.

2) Apply thin layer of talc powder to entire surface to eliminate any tack. 


\section{APPENDIX B}

\section{Signal Modulation Algorithm code:}

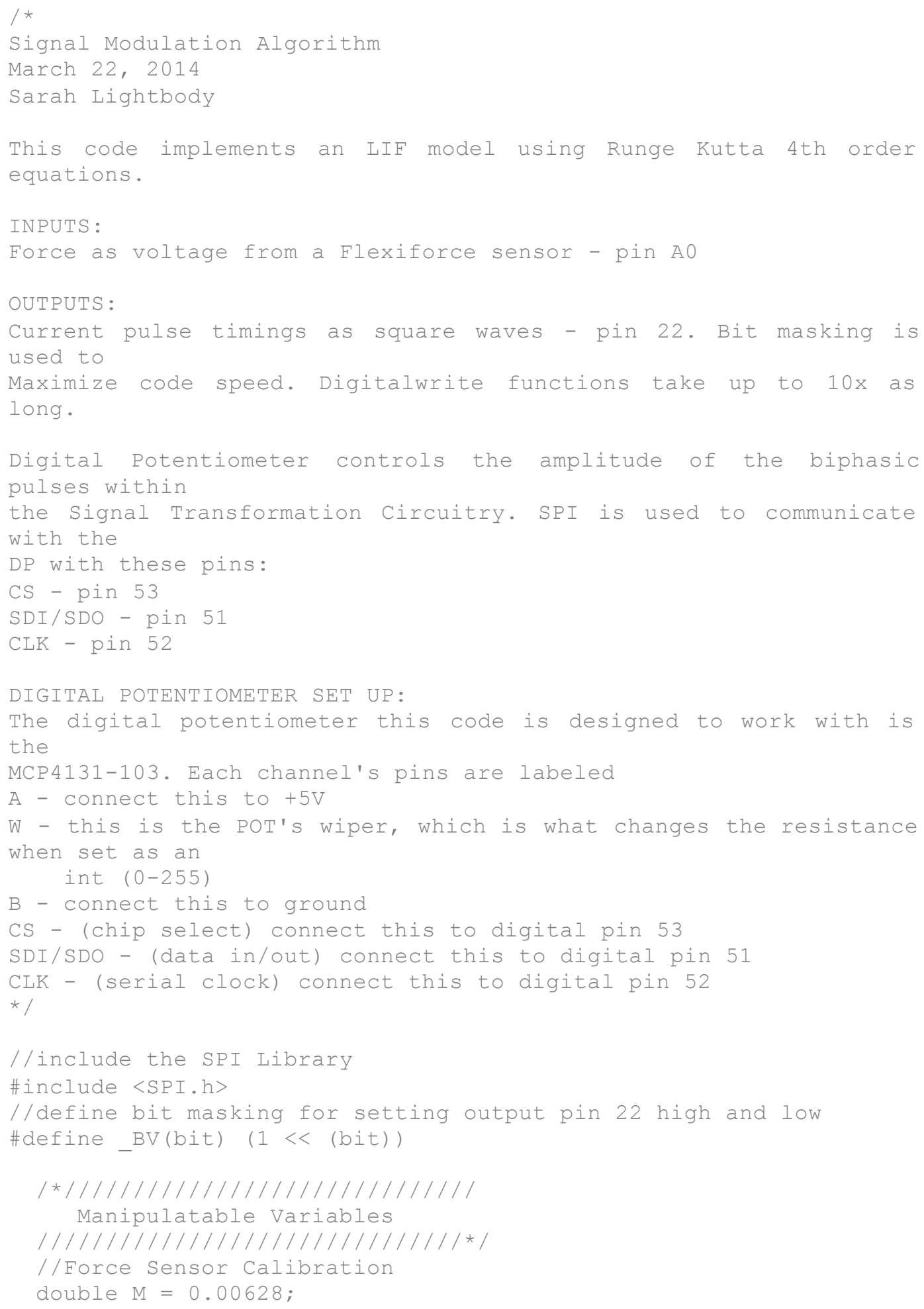




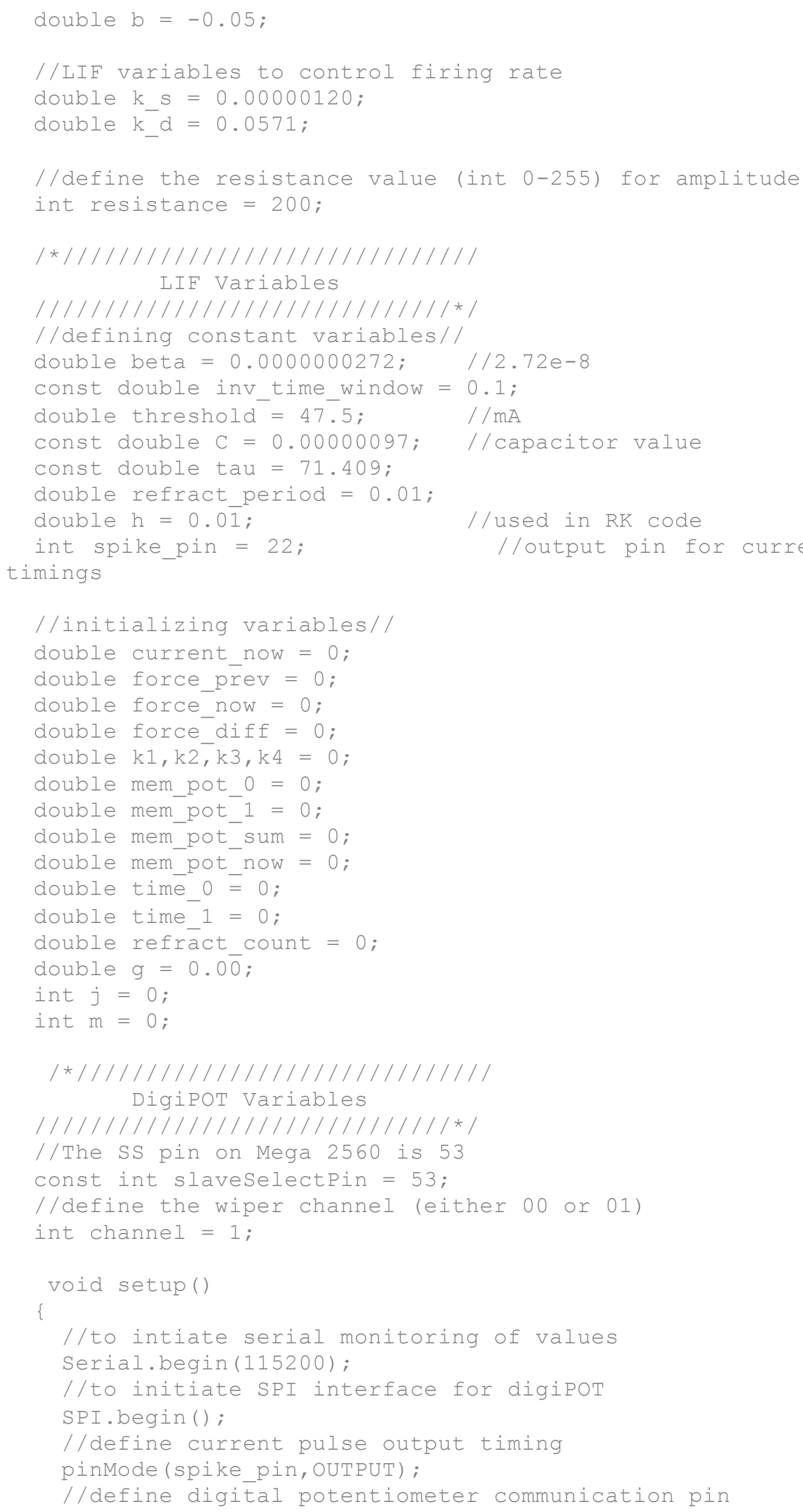




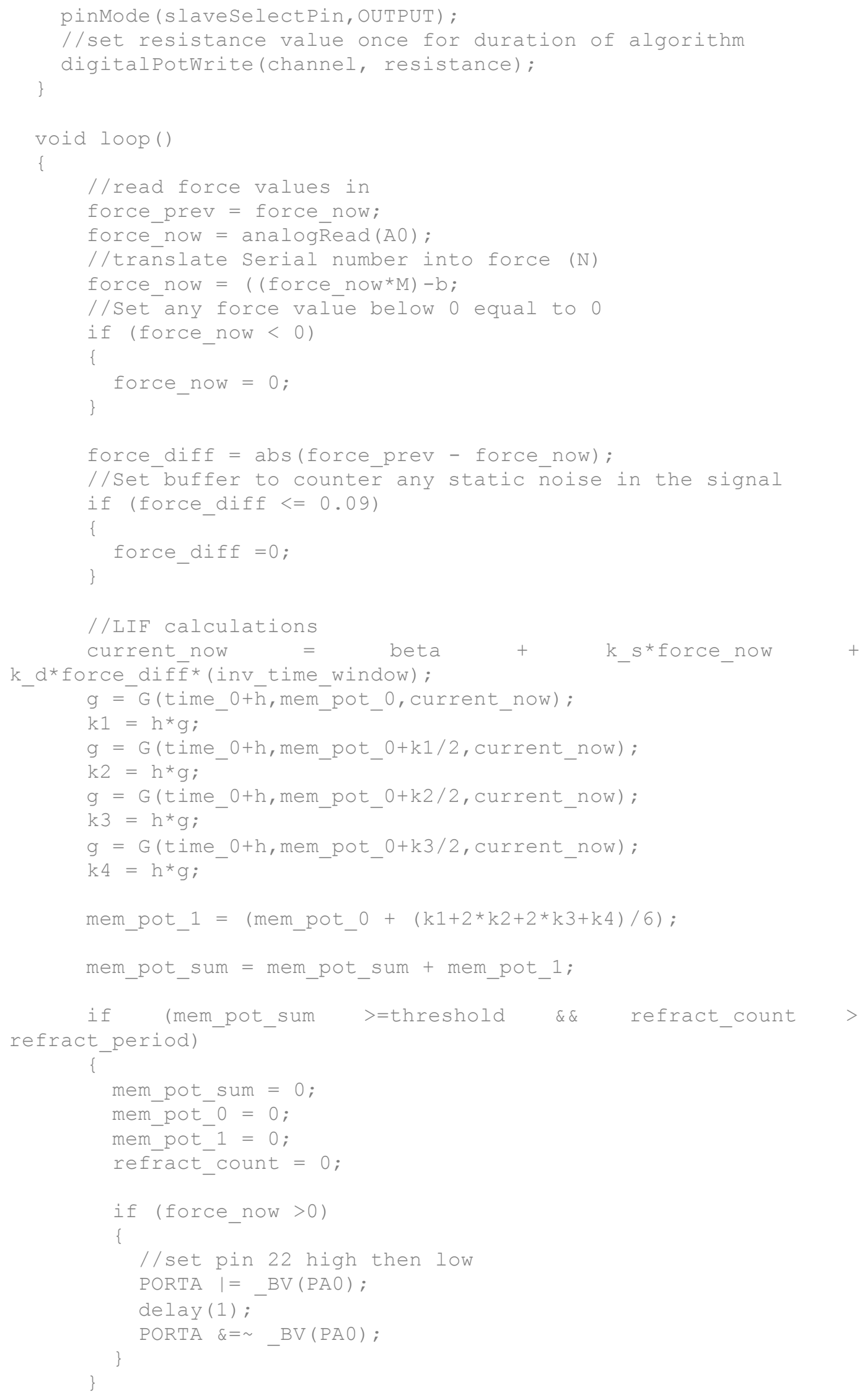




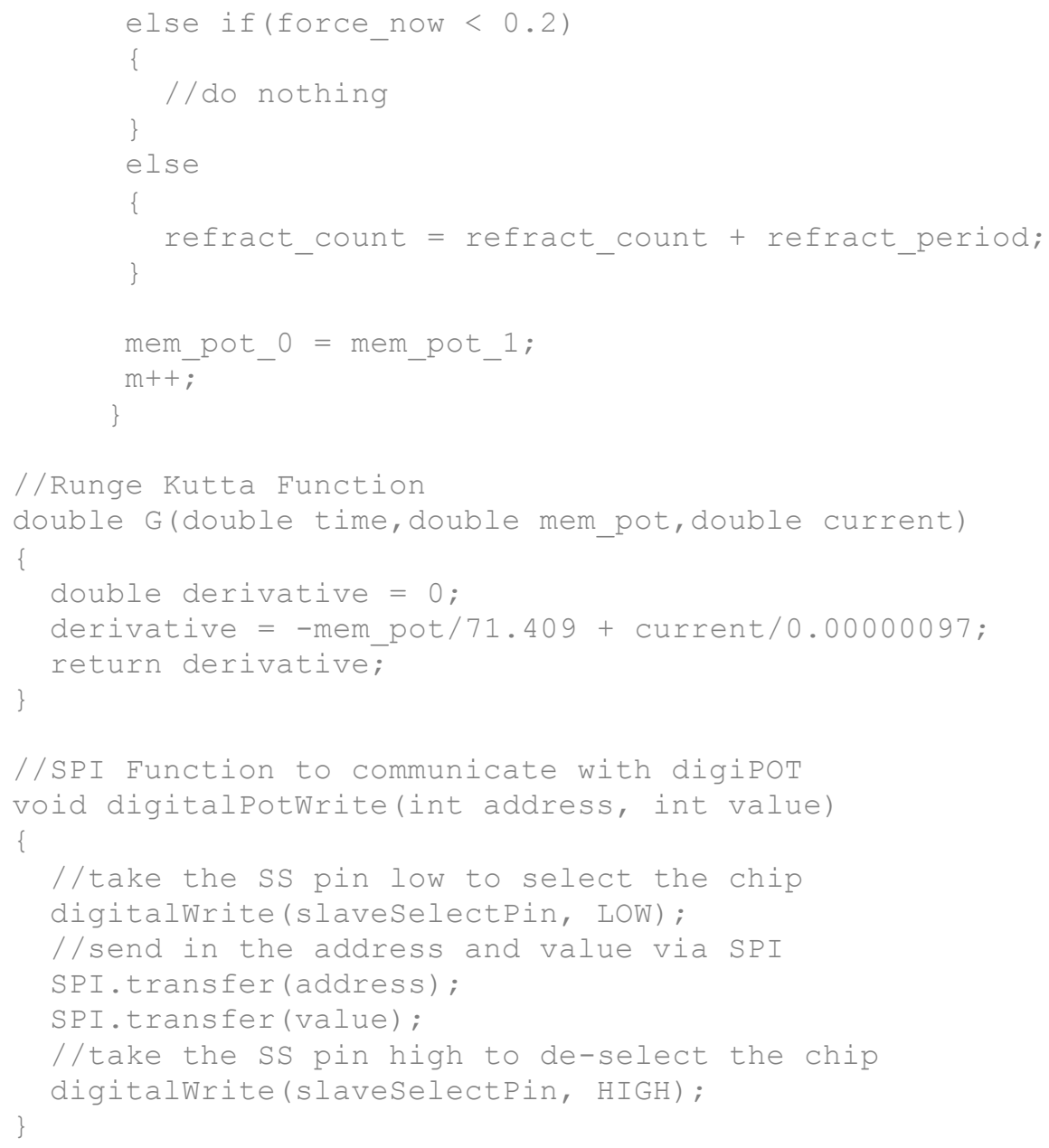




\section{APPENDIX C}

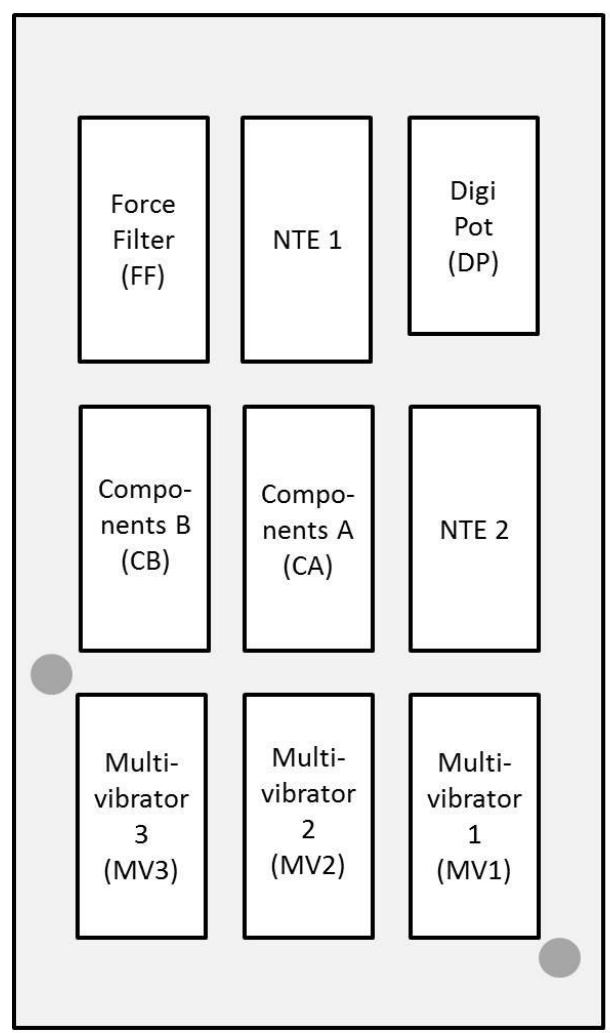

TOP

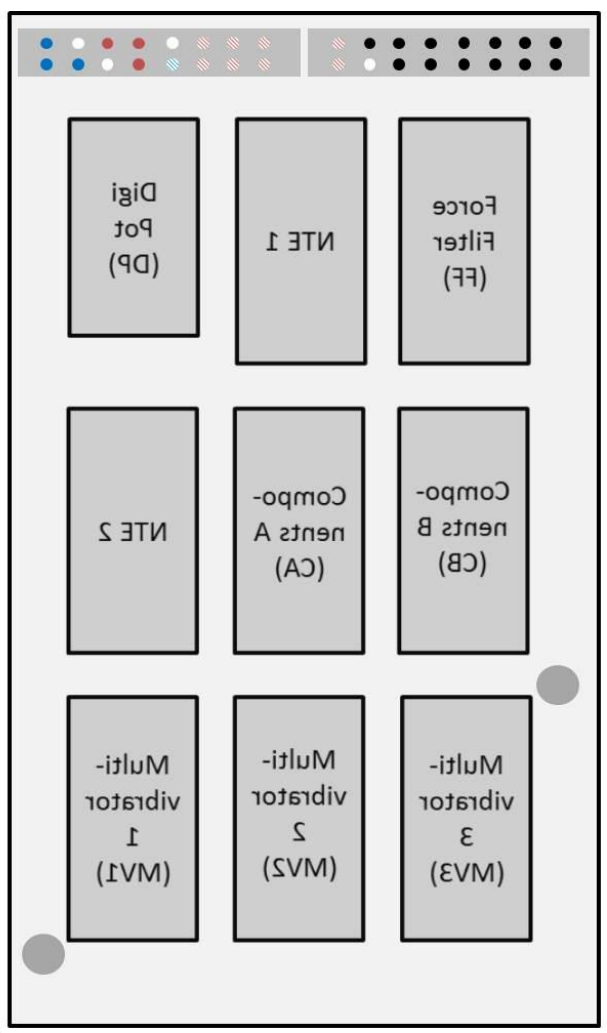

BOTTOM

Figure 1D: Schematic layout of the Signal Transformation Circuitry on a prototype board. The left image shows the board from a top down perspective, while the right image shows the underside of the board. Grey circles indicate where standoff's are attached to affix the circuit board to the power board (Figure 3D). 
Signal Transformation Circuitry Prototype Board Physical Layout
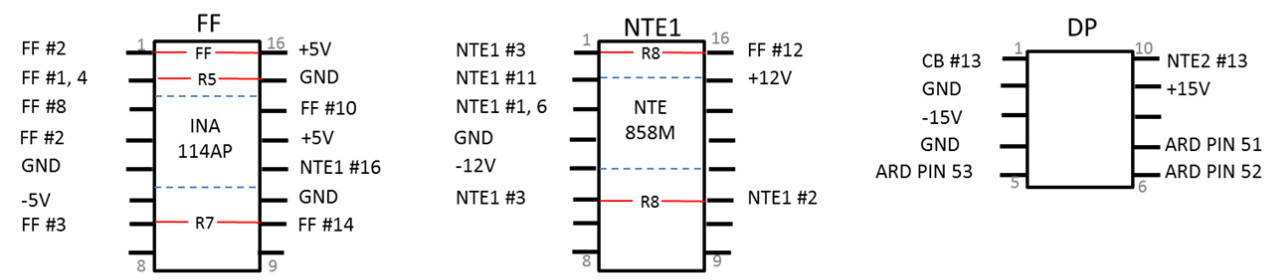

$\mathrm{R} 1=1.1 \mathrm{k} \Omega$

$\mathrm{R} 2=2.0 \mathrm{k} \Omega$

$\mathrm{R} 3=1 \mathrm{k} \Omega$

$\mathrm{R} 4=500 \Omega$

$\mathrm{R} 5=3.6 \mathrm{k} \Omega$

MV1 \#7 $\quad 1$ CB 16 NTE2 \#2

\begin{tabular}{ll|l|l} 
MV2 \#6 & R5 \\
NTE2 \#1 & R5 & NTE2 23 \\
NT6 & NTE2 22
\end{tabular}

NTE2 \#1
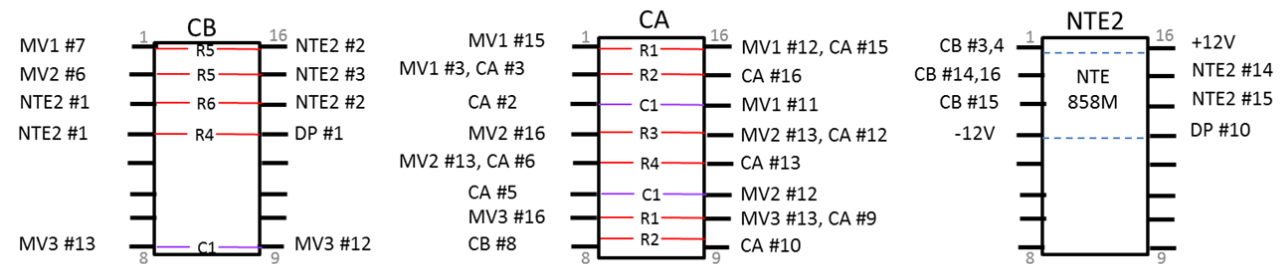

$\mathrm{R} 6=56 \mathrm{k} \Omega$

$\mathrm{R} 7=8 \mathrm{k} \Omega$

$\mathrm{R} 8=10 \mathrm{k} \Omega$

$\mathrm{C} 1=0.1 \mathrm{uF}$

$\mathrm{C} 2=47 \mathrm{nF}$
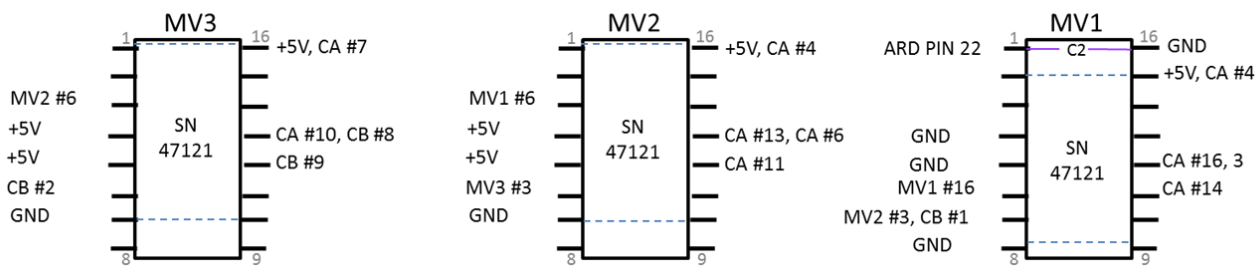

Figure 2D: Wire wrapping map for the Signal Transformation Circuitry prototype board. Red lines denote resistors, purple lines denote capacitors, and dotted lines denote areas dedicated to IC chips. The values of all components are listed in the legend to the right. The abbreviated names of each socket are mapped to the components in Figure 1D. 


\section{Signal Transformation Circuitry Power Board Physical Layout}

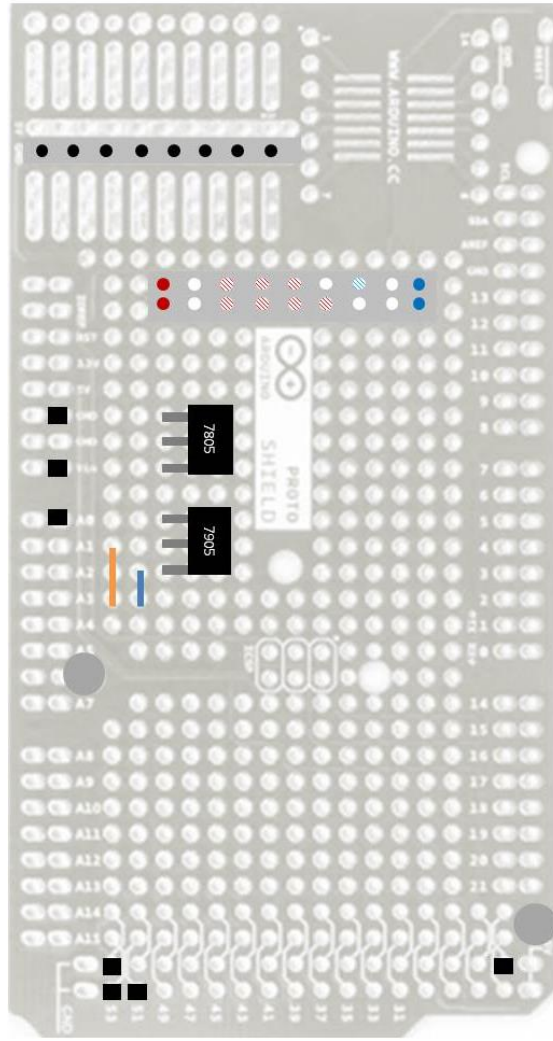

TOP

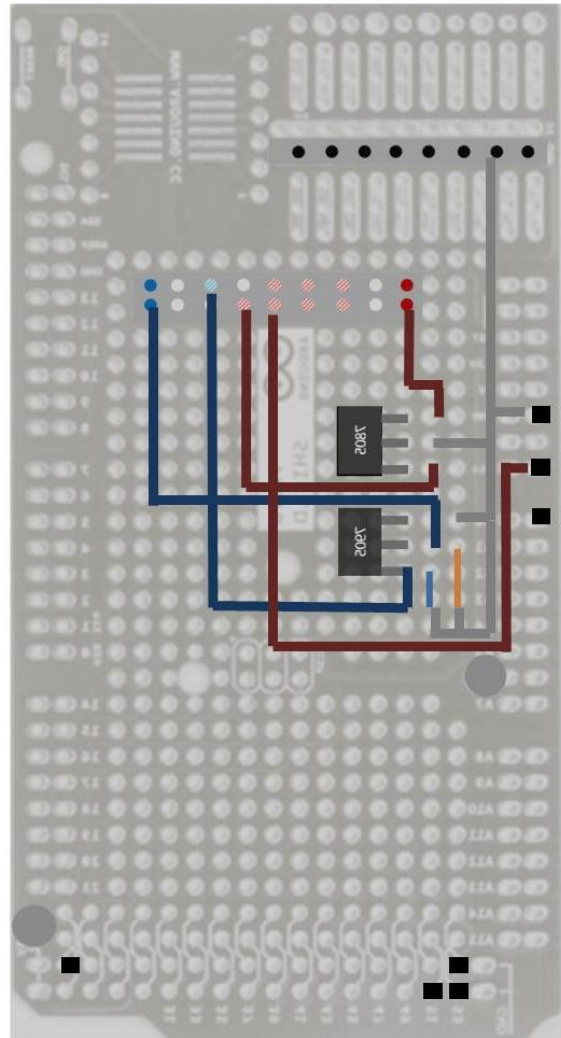

BOTTOM
- $=+12 \mathrm{~V}$

$=+5 \mathrm{~V}$

- $=-12 \mathrm{~V}$

$=-5 \mathrm{~V}$

- = GND

= Open pin

- Header

$=2.2$ uF capacitor

$=1.0$ uF capacitor

Figure 3D: Schematic for Signal Transformation Circuitry Power Board. The board used here is a blank shield for the Arduino Mega 2650 (Mega Proto PCB R3; Arduino, Torino, Italy). Two voltage regulators, $+5 \mathrm{~V}(7805)$ and $-5 \mathrm{~V}(7905)$ are pictured from top to bottom, respectively. Grey circles denote where standoff's can be placed to match the Signal Transformation Circuitry Prototype Board. The use of this shield allows for direct access to the Arduino pins while protecting the processing board. It is important to note that the type of capacitors needed is solid tantalum. If these are not available, $25 \mathrm{uF}$ aluminum electrolytic can be substituted. The legend (top right) denotes all other symbols. 


\section{APPENDIX D}

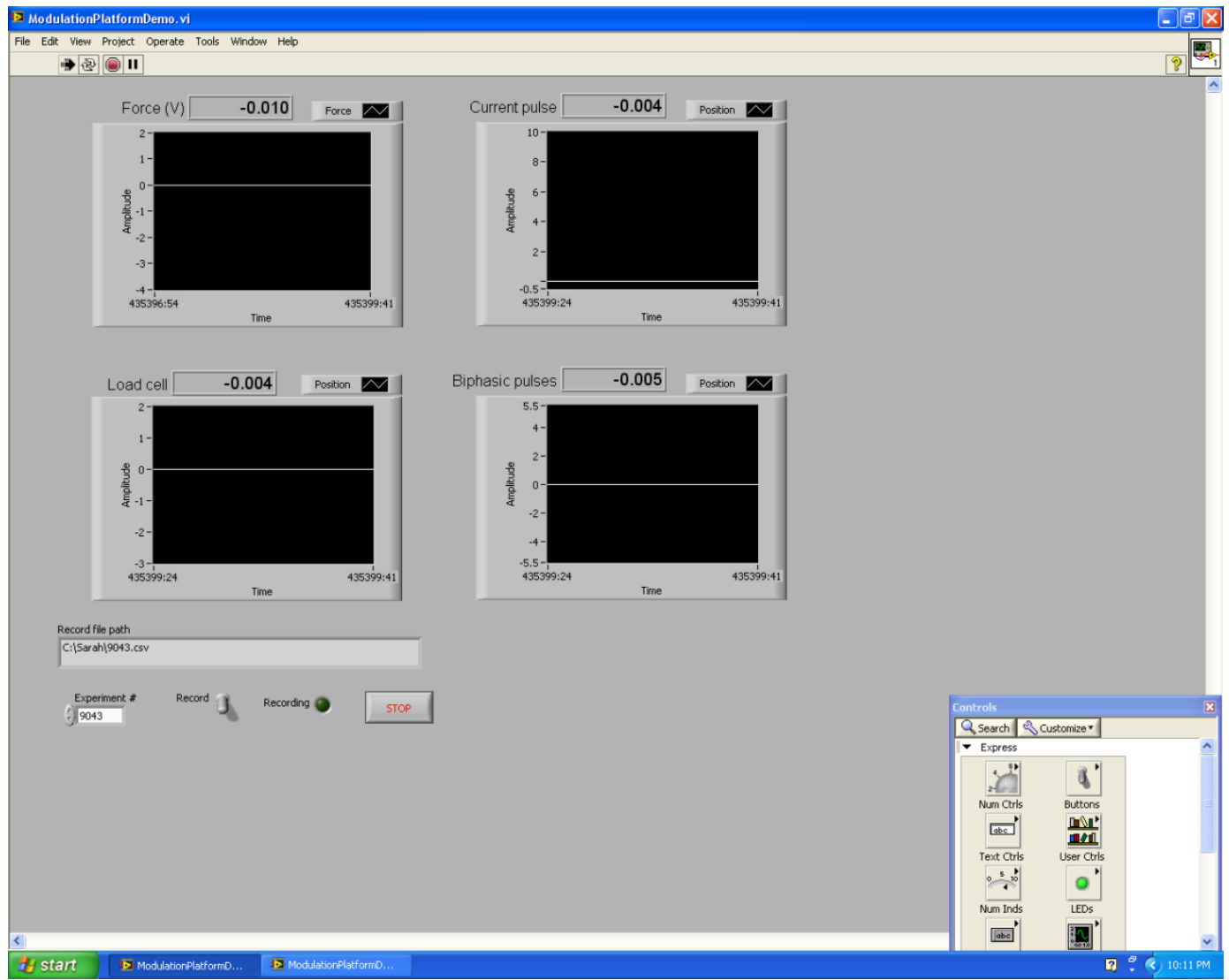

\begin{tabular}{|l|l|}
\hline \multicolumn{1}{|c|}{ Tactile Modulation Platform } & \multicolumn{1}{c|}{ NI Breakout Board Connection } \\
\hline Force & J68 (A1 0) \\
\hline Current Pulse Timings & J33 (A1 1) \\
\hline Biphasic Pulses & J65 (A1 2) \\
\hline Load Cell & J30 (A1 3) \\
\hline Ground & J67 (A1 GND) \\
\hline
\end{tabular}




\section{APPENDIX E}

\begin{tabular}{|c|c|c|c|c|c|c|c|c|c|}
\hline$\alpha$ & Amplitude & $\alpha$ & Amplitude & $\alpha$ & Amplitude & $\alpha$ & Amplitude & $\alpha$ & Amplitude \\
\hline 1 & 0.2 & 52 & 3.2 & 103 & 6.2 & 154 & 9.2 & 205 & 12.2 \\
\hline 2 & 0.3 & 53 & 3.3 & 104 & 6.3 & 155 & 9.3 & 206 & 12.3 \\
\hline 3 & 0.3 & 54 & 3.3 & 105 & 6.3 & 156 & 9.3 & 207 & 12.3 \\
\hline 4 & 0.4 & 55 & 3.4 & 106 & 6.4 & 157 & 9.4 & 208 & 12.4 \\
\hline 5 & 0.4 & 56 & 3.4 & 107 & 6.4 & 158 & 9.4 & 209 & 12.4 \\
\hline 6 & 0.5 & 57 & 3.5 & 108 & 6.5 & 159 & 9.5 & 210 & 12.5 \\
\hline 7 & 0.6 & 58 & 3.6 & 109 & 6.6 & 160 & 9.5 & 211 & 12.5 \\
\hline 8 & 0.6 & 59 & 3.6 & 110 & 6.6 & 161 & 9.6 & 212 & 12.6 \\
\hline 9 & 0.7 & 60 & 3.7 & 111 & 6.7 & 162 & 9.7 & 213 & 12.7 \\
\hline 10 & 0.7 & 61 & 3.7 & 112 & 6.7 & 163 & 9.7 & 214 & 12.7 \\
\hline 11 & 0.8 & 62 & 3.8 & 113 & 6.8 & 164 & 9.8 & 215 & 12.8 \\
\hline 12 & 0.8 & 63 & 3.8 & 114 & 6.8 & 165 & 9.8 & 216 & 12.8 \\
\hline 13 & 0.9 & 64 & 3.9 & 115 & 6.9 & 166 & 9.9 & 217 & 12.9 \\
\hline 14 & 1.0 & 65 & 4.0 & 116 & 7.0 & 167 & 10.0 & 218 & 13.0 \\
\hline 15 & 1.0 & 66 & 4.0 & 117 & 7.0 & 168 & 10.0 & 219 & 13.0 \\
\hline 16 & 1.1 & 67 & 4.1 & 118 & 7.1 & 169 & 10.1 & 220 & 13.1 \\
\hline 17 & 1.1 & 68 & 4.1 & 119 & 7.1 & 170 & 10.1 & 221 & 13.1 \\
\hline 18 & 1.2 & 69 & 4.2 & 120 & 7.2 & 171 & 10.2 & 222 & 13.2 \\
\hline 19 & 1.3 & 70 & 4.3 & 121 & 7.3 & 172 & 10.3 & 223 & 13.3 \\
\hline 20 & 1.3 & 71 & 4.3 & 122 & 7.3 & 173 & 10.3 & 224 & 13.3 \\
\hline 21 & 1.4 & 72 & 4.4 & 123 & 7.4 & 174 & 10.4 & 225 & 13.4 \\
\hline 22 & 1.4 & 73 & 4.4 & 124 & 7.4 & 175 & 10.4 & 226 & 13.4 \\
\hline 23 & 1.5 & 74 & 4.5 & 125 & 7.5 & 176 & 10.5 & 227 & 13.5 \\
\hline 24 & 1.6 & 75 & 4.6 & 126 & 7.5 & 177 & 10.5 & 228 & 13.5 \\
\hline 25 & 1.6 & 76 & 4.6 & 127 & 7.6 & 178 & 10.6 & 229 & 13.6 \\
\hline 26 & 1.7 & 77 & 4.7 & 128 & 7.7 & 179 & 10.7 & 230 & 13.7 \\
\hline 27 & 1.7 & 78 & 4.7 & 129 & 7.7 & 180 & 10.7 & 231 & 13.7 \\
\hline 28 & 1.8 & 79 & 4.8 & 130 & 7.8 & 181 & 10.8 & 232 & 13.8 \\
\hline 29 & 1.8 & 80 & 4.8 & 131 & 7.8 & 182 & 10.8 & 233 & 13.8 \\
\hline 30 & 1.9 & 81 & 4.9 & 132 & 7.9 & 183 & 10.9 & 234 & 13.9 \\
\hline 31 & 2.0 & 82 & 5.0 & 133 & 8.0 & 184 & 11.0 & 235 & 14.0 \\
\hline 32 & 2.0 & 83 & 5.0 & 134 & 8.0 & 185 & 11.0 & 236 & 14.0 \\
\hline 33 & 2.1 & 84 & 5.1 & 135 & 8.1 & 186 & 11.1 & 237 & 14.1 \\
\hline 34 & 2.1 & 85 & 5.1 & 136 & 8.1 & 187 & 11.1 & 238 & 14.1 \\
\hline 35 & 2.2 & 86 & 5.2 & 137 & 8.2 & 188 & 11.2 & 239 & 14.2 \\
\hline 36 & 2.3 & 87 & 5.3 & 138 & 8.3 & 189 & 11.3 & 240 & 14.3 \\
\hline 37 & 2.3 & 88 & 5.3 & 139 & 8.3 & 190 & 11.3 & 241 & 14.3 \\
\hline 38 & 2.4 & 89 & 5.4 & 140 & 8.4 & 191 & 11.4 & 242 & 14.4 \\
\hline 39 & 2.4 & 90 & 5.4 & 141 & 8.4 & 192 & 11.4 & 243 & 14.4 \\
\hline 40 & 2.5 & 91 & 5.5 & 142 & 8.5 & 193 & 11.5 & 244 & 14.5 \\
\hline 41 & 2.6 & 92 & 5.6 & 143 & 8.5 & 194 & 11.5 & 245 & 14.5 \\
\hline 42 & 2.6 & 93 & 5.6 & 144 & 8.6 & 195 & 11.6 & 246 & 14.6 \\
\hline 43 & 2.7 & 94 & 5.7 & 145 & 8.7 & 196 & 11.7 & 247 & 14.7 \\
\hline 44 & 2.7 & 95 & 5.7 & 146 & 8.7 & 197 & 11.7 & 248 & 14.7 \\
\hline 45 & 2.8 & 96 & 5.8 & 147 & 8.8 & 198 & 11.8 & 249 & 14.8 \\
\hline 46 & 2.8 & 97 & 5.8 & 148 & 8.8 & 199 & 11.8 & 250 & 14.8 \\
\hline 47 & 2.9 & 98 & 5.9 & 149 & 8.9 & 200 & 11.9 & 251 & 14.9 \\
\hline 48 & 3.0 & 99 & 6.0 & 150 & 9.0 & 201 & 12.0 & 252 & 15.0 \\
\hline 49 & 3.0 & 100 & 6.0 & 151 & 9.0 & 202 & 12.0 & 253 & 15.0 \\
\hline 50 & 3.1 & 101 & 6.1 & 152 & 9.1 & 203 & 12.1 & 254 & 15.1 \\
\hline 51 & 3.1 & 102 & 6.1 & 153 & 9.1 & 204 & 12.1 & 255 & 15.1 \\
\hline
\end{tabular}




\begin{tabular}{|c|c|c|c|c|c|c|c|c|c|c|c|c|c|c|}
\hline \multicolumn{15}{|c|}{ Applied Current Using Electrode with 10, 4 kiloOhm impedance } \\
\hline $\begin{array}{c}\text { Voltage } \\
( \pm V)\end{array}$ & $\begin{array}{c}\text { Current } \\
10 \mathrm{kOhm}( \pm \mathrm{mA})\end{array}$ & $\begin{array}{c}\text { Current } \\
4 \mathrm{kOhm}( \pm \mathrm{mA})\end{array}$ & $\pm \mathbf{V}$ & $\pm m A$ & $\pm m A$ & $\pm V$ & $\pm m A$ & $\pm \mathrm{mA}$ & $\pm V$ & $\pm m A$ & $\pm m A$ & $\pm V$ & $\pm \mathrm{mA}$ & $\pm m A$ \\
\hline 0.20 & 0.02 & 0.05 & 3.58 & 0.36 & 0.89 & 6.95 & 0.70 & 1.74 & 10.33 & 1.03 & 2.58 & 13.70 & 1.37 & 3.43 \\
\hline 0.26 & 0.03 & 0.06 & 3.63 & 0.36 & 0.91 & 7.01 & 0.70 & 1.75 & 10.39 & 1.04 & 2.60 & 13.76 & 1.38 & 3.44 \\
\hline 0.32 & 0.03 & 0.08 & 3.69 & 0.37 & 0.92 & 7.07 & 0.71 & 1.77 & 10.44 & 1.04 & 2.61 & 13.82 & 1.38 & 3.45 \\
\hline 0.37 & 0.04 & 0.09 & 3.75 & 0.38 & 0.94 & 7.13 & 0.71 & 1.78 & 10.50 & 1.05 & 2.63 & 13.88 & 1.39 & 3.47 \\
\hline 0.43 & 0.04 & 0.11 & 3.81 & 0.38 & 0.95 & 7.18 & 0.72 & 1.80 & 10.56 & 1.06 & 2.64 & 13.94 & 1.39 & 3.48 \\
\hline 0.49 & 0.05 & 0.12 & 3.87 & 0.39 & 0.97 & 7.24 & 0.72 & 1.81 & 10.62 & 1.06 & 2.65 & 13.99 & 1.40 & 3.50 \\
\hline 0.55 & 0.05 & 0.14 & 3.92 & 0.39 & 0.98 & 7.30 & 0.73 & 1.83 & 10.68 & 1.07 & 2.67 & 14.05 & 1.41 & 3.51 \\
\hline 0.61 & 0.06 & 0.15 & 3.98 & 0.40 & 1.00 & 7.36 & 0.74 & 1.84 & 10.73 & 1.07 & 2.68 & 14.11 & 1.41 & 3.53 \\
\hline 0.67 & 0.07 & 0.17 & 4.04 & 0.40 & 1.01 & 7.42 & 0.74 & 1.85 & 10.79 & 1.08 & 2.70 & 14.17 & 1.42 & 3.54 \\
\hline 0.72 & 0.07 & 0.18 & 4.10 & 0.41 & 1.02 & 7.48 & 0.75 & 1.87 & 10.85 & 1.09 & 2.71 & 14.23 & 1.42 & 3.56 \\
\hline 0.78 & 0.08 & 0.20 & 4.16 & 0.42 & 1.04 & 7.53 & 0.75 & 1.88 & 10.91 & 1.09 & 2.73 & 14.28 & 1.43 & 3.57 \\
\hline 0.84 & 0.08 & 0.21 & 4.22 & 0.42 & 1.05 & 7.59 & 0.76 & 1.90 & 10.97 & 1.10 & 2.74 & 14.34 & 1.43 & 3.59 \\
\hline 0.90 & 0.09 & 0.22 & 4.27 & 0.43 & 1.07 & 7.65 & 0.76 & 1.91 & 11.03 & 1.10 & 2.76 & 14.40 & 1.44 & 3.60 \\
\hline 0.96 & 0.10 & 0.24 & 4.33 & 0.43 & 1.08 & 7.71 & 0.77 & 1.93 & 11.08 & 1.11 & 2.77 & 14.46 & 1.45 & 3.61 \\
\hline 1.01 & 0.10 & 0.25 & 4.39 & 0.44 & 1.10 & 7.77 & 0.78 & 1.94 & 11.14 & 1.11 & 2.79 & 14.52 & 1.45 & 3.63 \\
\hline 1.07 & 0.11 & 0.27 & 4.45 & 0.44 & 1.11 & 7.82 & 0.78 & 1.96 & 11.20 & 1.12 & 2.80 & 14.58 & 1.46 & 3.64 \\
\hline 1.13 & 0.11 & 0.28 & 4.51 & 0.45 & 1.13 & 7.88 & 0.79 & 1.97 & 11.26 & 1.13 & 2.81 & 14.63 & 1.46 & 3.66 \\
\hline 1.19 & 0.12 & 0.30 & 4.57 & 0.46 & 1.14 & 7.94 & 0.79 & 1.99 & 11.32 & 1.13 & 2.83 & 14.69 & 1.47 & 3.67 \\
\hline 1.25 & 0.12 & 0.31 & 4.62 & 0.46 & 1.16 & 8.00 & 0.80 & 2.00 & 11.37 & 1.14 & 2.84 & 14.75 & 1.47 & 3.69 \\
\hline 1.31 & 0.13 & 0.33 & 4.68 & 0.47 & 1.17 & 8.06 & 0.81 & 2.01 & 11.43 & 1.14 & 2.86 & 14.81 & 1.48 & 3.70 \\
\hline 1.36 & 0.14 & 0.34 & 4.74 & 0.47 & 1.18 & 8.12 & 0.81 & 2.03 & 11.49 & 1.15 & 2.87 & 14.87 & 1.49 & 3.72 \\
\hline 1.42 & 0.14 & 0.36 & 4.80 & 0.48 & 1.20 & 8.17 & 0.82 & 2.04 & 11.55 & 1.15 & 2.89 & 14.92 & 1.49 & 3.73 \\
\hline 1.48 & 0.15 & 0.37 & 4.86 & 0.49 & 1.21 & 8.23 & 0.82 & 2.06 & 11.61 & 1.16 & 2.90 & 14.98 & 1.50 & 3.75 \\
\hline 1.54 & 0.15 & 0.38 & 4.91 & 0.49 & 1.23 & 8.29 & 0.83 & 2.07 & 11.67 & 1.17 & 2.92 & 15.04 & 1.50 & 3.76 \\
\hline 1.60 & 0.16 & 0.40 & 4.97 & 0.50 & 1.24 & 8.35 & 0.83 & 2.09 & 11.72 & 1.17 & 2.93 & & & \\
\hline 1.66 & 0.17 & 0.41 & 5.03 & 0.50 & 1.26 & 8.41 & 0.84 & 2.10 & 11.78 & 1.18 & 2.95 & & & \\
\hline 1.71 & 0.17 & 0.43 & 5.09 & 0.51 & 1.27 & 8.46 & 0.85 & 2.12 & 11.84 & 1.18 & 2.96 & & & \\
\hline 1.77 & 0.18 & 0.44 & 5.15 & 0.51 & 1.29 & 8.52 & 0.85 & 2.13 & 11.90 & 1.19 & 2.97 & & & \\
\hline 1.83 & 0.18 & 0.46 & 5.21 & 0.52 & 1.30 & 8.58 & 0.86 & 2.15 & 11.96 & 1.20 & 2.99 & & & \\
\hline 1.89 & 0.19 & 0.47 & 5.26 & 0.53 & 1.32 & 8.64 & 0.86 & 2.16 & 12.01 & 1.20 & 3.00 & & & \\
\hline 1.95 & 0.19 & 0.49 & 5.32 & 0.53 & 1.33 & 8.70 & 0.87 & 2.17 & 12.07 & 1.21 & 3.02 & & & \\
\hline 2.00 & 0.20 & 0.50 & 5.38 & 0.54 & 1.34 & 8.76 & 0.88 & 2.19 & 12.13 & 1.21 & 3.03 & & & \\
\hline 2.06 & 0.21 & 0.52 & 5.44 & 0.54 & 1.36 & 8.81 & 0.88 & 2.20 & 12.19 & 1.22 & 3.05 & & & \\
\hline 2.12 & 0.21 & 0.53 & 5.50 & 0.55 & 1.37 & 8.87 & 0.89 & 2.22 & 12.25 & 1.22 & 3.06 & & & \\
\hline 2.18 & 0.22 & 0.54 & 5.55 & 0.56 & 1.39 & 8.93 & 0.89 & 2.23 & 12.31 & 1.23 & 3.08 & & & \\
\hline 2.24 & 0.22 & 0.56 & 5.61 & 0.56 & 1.40 & 8.99 & 0.90 & 2.25 & 12.36 & 1.24 & 3.09 & & & \\
\hline 2.30 & 0.23 & 0.57 & 5.67 & 0.57 & 1.42 & 9.05 & 0.90 & 2.26 & 12.42 & 1.24 & 3.11 & & & \\
\hline 2.35 & 0.24 & 0.59 & 5.73 & 0.57 & 1.43 & 9.10 & 0.91 & 2.28 & 12.48 & 1.25 & 3.12 & & & \\
\hline 2.41 & 0.24 & 0.60 & 5.79 & 0.58 & 1.45 & 9.16 & 0.92 & 2.29 & 12.54 & 1.25 & 3.13 & & & \\
\hline 2.47 & 0.25 & 0.62 & 5.85 & 0.58 & 1.46 & 9.22 & 0.92 & 2.31 & 12.60 & 1.26 & 3.15 & & & \\
\hline 2.53 & 0.25 & 0.63 & 5.90 & 0.59 & 1.48 & 9.28 & 0.93 & 2.32 & 12.65 & 1.27 & 3.16 & & & \\
\hline 2.59 & 0.26 & 0.65 & 5.96 & 0.60 & 1.49 & 9.34 & 0.93 & 2.33 & 12.71 & 1.27 & 3.18 & & & \\
\hline 2.64 & 0.26 & 0.66 & 6.02 & 0.60 & 1.51 & 9.40 & 0.94 & 2.35 & 12.77 & 1.28 & 3.19 & & & \\
\hline 2.70 & 0.27 & 0.68 & 6.08 & 0.61 & 1.52 & 9.45 & 0.95 & 2.36 & 12.83 & 1.28 & 3.21 & & & \\
\hline 2.76 & 0.28 & 0.69 & 6.14 & 0.61 & 1.53 & 9.51 & 0.95 & 2.38 & 12.89 & 1.29 & 3.22 & & & \\
\hline 2.82 & 0.28 & 0.70 & 6.19 & 0.62 & 1.55 & 9.57 & 0.96 & 2.39 & 12.95 & 1.29 & 3.24 & & & \\
\hline 2.88 & 0.29 & 0.72 & 6.25 & 0.63 & 1.56 & 9.63 & 0.96 & 2.41 & 13.00 & 1.30 & 3.25 & & & \\
\hline 2.94 & 0.29 & 0.73 & 6.31 & 0.63 & 1.58 & 9.69 & 0.97 & 2.42 & 13.06 & 1.31 & 3.27 & & & \\
\hline 2.99 & 0.30 & 0.75 & 6.37 & 0.64 & 1.59 & 9.74 & 0.97 & 2.44 & 13.12 & 1.31 & 3.28 & & & \\
\hline 3.05 & 0.31 & 0.76 & 6.43 & 0.64 & 1.61 & 9.80 & 0.98 & 2.45 & 13.18 & 1.32 & 3.29 & & & \\
\hline 3.11 & 0.31 & 0.78 & 6.49 & 0.65 & 1.62 & 9.86 & 0.99 & 2.47 & 13.24 & 1.32 & 3.31 & & & \\
\hline 3.17 & 0.32 & 0.79 & 6.54 & 0.65 & 1.64 & 9.92 & 0.99 & 2.48 & 13.30 & 1.33 & 3.32 & & & \\
\hline 3.23 & 0.32 & 0.81 & 6.60 & 0.66 & 1.65 & 9.98 & 1.00 & 2.49 & 13.35 & 1.34 & 3.34 & & & \\
\hline 3.28 & 0.33 & 0.82 & 6.66 & 0.67 & 1.67 & 10.04 & 1.00 & 2.51 & 13.41 & 1.34 & 3.35 & & & \\
\hline 3.34 & 0.33 & 0.84 & 6.72 & 0.67 & 1.68 & 10.09 & 1.01 & 2.52 & 13.47 & 1.35 & 3.37 & & & \\
\hline 3.40 & 0.34 & 0.85 & 6.78 & 0.68 & 1.69 & 10.15 & 1.02 & 2.54 & 13.53 & 1.35 & 3.38 & & & \\
\hline 3.46 & 0.35 & 0.86 & 6.83 & 0.68 & 1.71 & 10.21 & 1.02 & 2.55 & 13.59 & 1.36 & 3.40 & & & \\
\hline 3.52 & 0.35 & 0.88 & 6.89 & 0.69 & 1.72 & 10.27 & 1.03 & 2.57 & 13.64 & 1.36 & 3.41 & & & \\
\hline
\end{tabular}




\section{APPENDIX F}

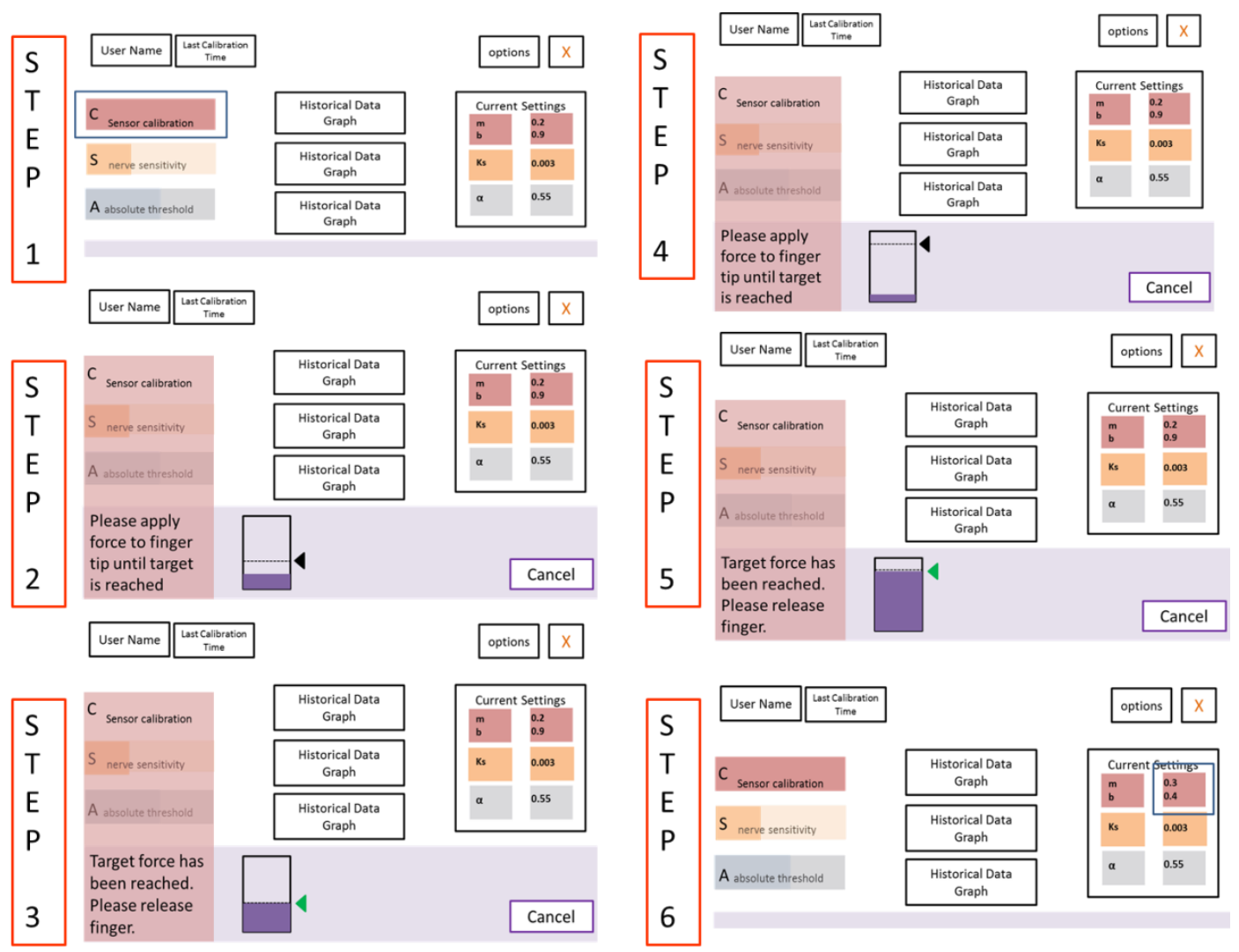

Figure 1F: A step by step walk through of the sensor calibration user interface. Step 1 shows the main screen, where the "sensor calibration" button is selected. Step 2 shows the user instructions for applying force at the finger force sensor until the target (shown with a dotted line and triangle) is reached (where the users applied force is relayed with a purple bar). Step 3 shows the event where the user reaches the target level, where the black triangle changes to green to indicate a reached target. Step 4 shows the next iteration of applied force, with exact instructions as Step 2. Step 5 again shows the user applying the correct amount of force. Enough iterations are used until an $R^{2}$ value $>0.9$ is reached. After this is accomplished, Step 6 shows the main screen again, but with updated parameter ( $m$ and b) values. 


\section{APPENDIX G}
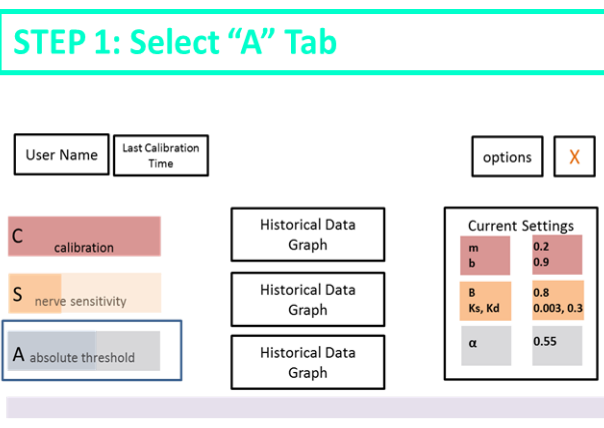

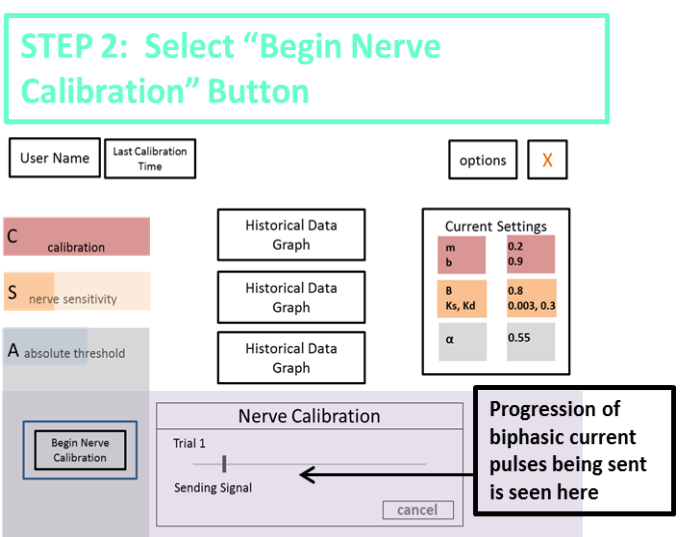

STEP 4: Notice updated setting
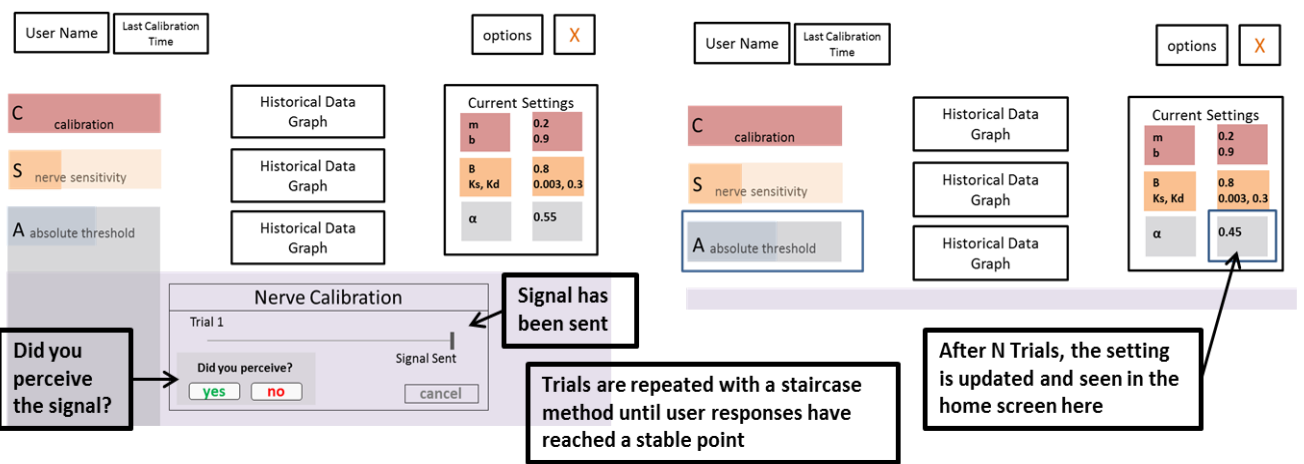

Figure 1G: A step by step walk through of the nerve absolute detection threshold user interface. Step 1 shows the main screen, where the "absolute threshold" button is selected. Step 2 shows the user that a signal is being sent to the nerve with the "Sending signal" info bar. The user has the option to cancel at any time via the cancel button. In Step 3, the signal has ended, denoted with the "Sending Sent" info bar, and the user is prompted to answer the yes or no question, "Did you perceive". A sequence of trials is repeated, according to a forced choice staircase methodology, until $n$ number of trials determines a threshold at which the user can just perceive the signal. 


\section{APPENDIX H}

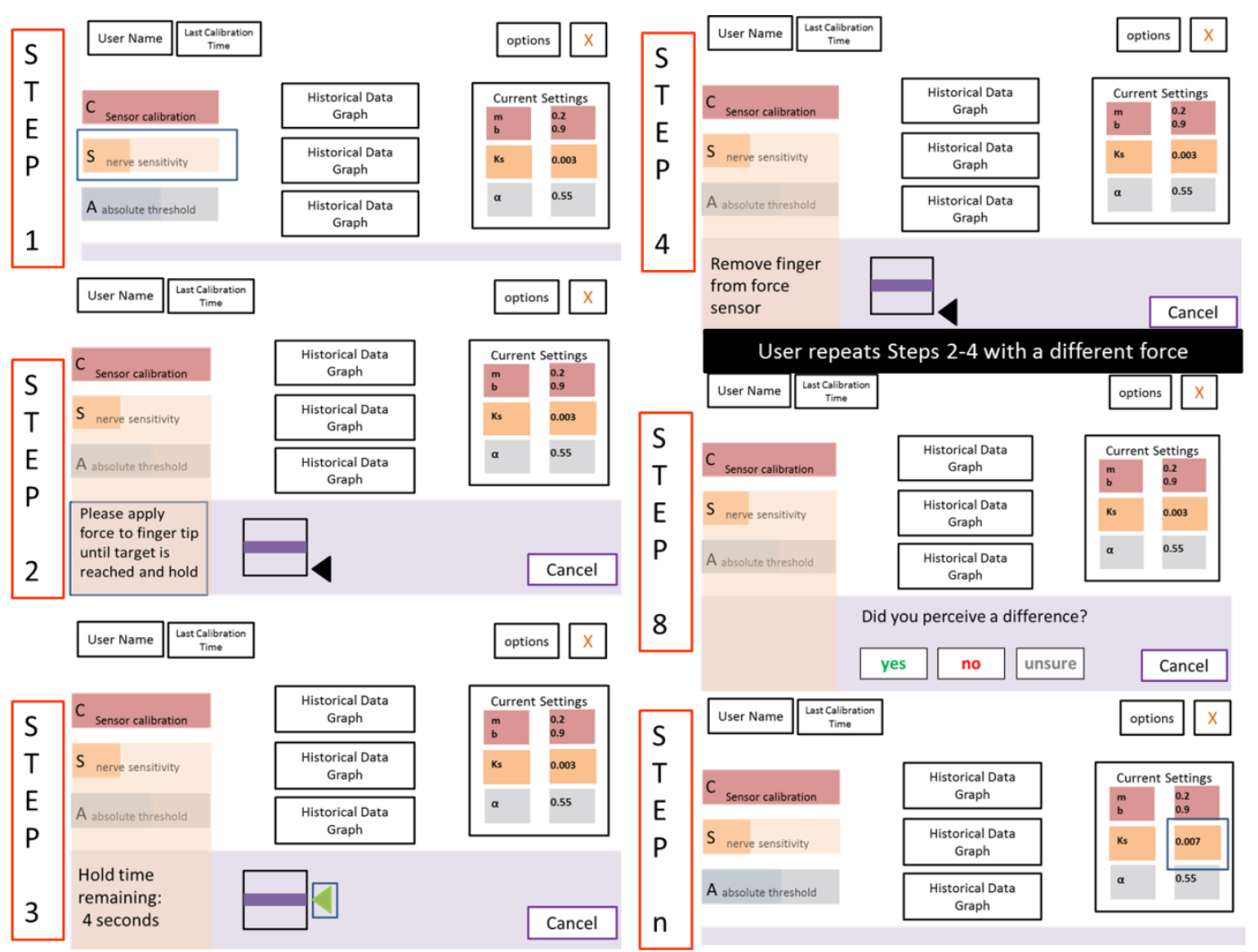

Figure 1H: A step by step walk through of the nerve sensitivity calibration user interface. Step 1 shows the main screen where the nerve sensitivity button is selected. Step 2 instructs the user to apply force to the fingertip until the target is reached (indicated by the purple bar). Step 3 shows that the user has reached the target (indicated by the green arrow) and is instructed to hold for 5 seconds (4 remaining). Step 4 indicates that the user has completed Step 3 and instructs to remove the finger from the load cell. The user then repeats this process for another force, but the target bar remains the same to prevent bias. Step 8 instructs the user to answer the question "Did you perceive a difference?" After $n$ steps, the parameter $k_{s}$ is updated. 